\title{
A Survey of Elliptic Cohomology
}

\section{Citation}

Lurie, Jacob. 2009. A survey of elliptic cohomology. In Algebraic Topology: The Abel Symposium 2007: Proceedings of the fourth Abel Symposium, Oslo, Norway, August 5-10, 2007, ed. N. A. Baas, E. M. Friedlander, B. Jahren, and P. A. Ostvaer, 219-278. Berlin: Springer.

\section{Published Version}

doi:10.1007/978-3-642-01200-6_9

\section{Permanent link}

http://nrs.harvard.edu/urn-3:HUL.InstRepos:8895113

\section{Terms of Use}

This article was downloaded from Harvard University's DASH repository, and is made available under the terms and conditions applicable to Other Posted Material, as set forth at http:// nrs.harvard.edu/urn-3:HUL.InstRepos:dash.current.terms-of-use\#LAA

\section{Share Your Story}

The Harvard community has made this article openly available.

Please share how this access benefits you. Submit a story.

Accessibility 


\section{A Survey of Elliptic Cohomology}

Jacob Lurie

Massachusetts Institute of Technology

This paper is an expository account of the relationship between elliptic cohomology and the emerging subject of derived algebraic geometry. We begin in $\S 1$ with an overview of the classical theory of elliptic cohomology. In $\S 2$ we review the theory of $E_{\infty}$-ring spectra and introduce the language of derived algebraic geometry. We apply this theory in $\S 3$, where we introduce the notion of an oriented group scheme and describe connection between oriented group schemes and equivariant cohomology theories. In $\S 4$ we sketch a proof of our main result, which relates the classical theory of elliptic cohomology to the classification of oriented elliptic curves. In $\S 5$ we discuss various applications of these ideas, many of which rely upon a special feature of elliptic cohomology which we call 2-equivariance.

The theory that we are going to describe lies at the intersection of homotopy theory and algebraic geometry. We have tried to make our exposition accessible to those who are not specialists in algebraic topology; however, we do assume the reader is familiar with the language of algebraic geometry, particularly with the theory of elliptic curves. In order to keep our account readable, we will gloss over many details, particularly where the use of higher category theory is required. A more comprehensive account of the material described here, with complete definitions and proofs, will be given in [21].

In carrying out the work described in this paper, I have benefitted from the ideas of many people. I would like to thank Matthew Ando, Mark Behrens, Brian Conrad, Chris Douglas, Dan Freed, Tyler Lawson, Haynes Miller, Jack Morava, Charles Rezk, John Rognes, Stephan Stolz, Neil Strickland, Peter Teichner, Bertrand Toën, and Gabriele Vezzosi for helpful discussions. I would also like to thank John Rognes, Scott Carnahan, and Peter Landweber for offering many corrections to earlier versions of this paper. Most of all, I would like to thank Mike Hopkins: many of the ideas described in this paper are based on his suggestions.

\section{Elliptic Cohomology}

\subsection{Cohomology Theories}

To any topological space $X$ one can associate the singular cohomology groups $A^{n}(X)=\mathrm{H}^{n}(X ; \mathbf{Z})$. These invariants have a number of good properties, which are neatly summarized by the following axioms (which, in a slightly modified form, are due to Eilenberg and Steenrod: see [11]):

(1) For each $n \in \mathbf{Z}, A^{n}$ is a contravariant functor from the category of pairs of topological spaces $(Y \subseteq X)$ to abelian groups. (We recover the absolute cohomology groups $A^{n}(X)$ by taking $Y=\emptyset$.)

(2) If $f: X^{\prime} \rightarrow X$ is a weak homotopy equivalence (in other words, if it induces a bijection $\pi_{0}\left(X^{\prime}\right) \rightarrow \pi_{0}(X)$ and an isomorphism $\pi_{n}\left(X^{\prime}, x\right) \rightarrow \pi_{n}(X, f x)$ for every $n>0$ and every base point $\left.x \in X^{\prime}\right)$, then the induced map $A^{n}(X) \rightarrow A^{n}\left(X^{\prime}\right)$ is an isomorphism.

(3) To every triple $Z \subseteq Y \subseteq X$, there is an associated long exact sequence

$$
\ldots \rightarrow A^{n}(X, Y) \rightarrow A^{n}(X, Z) \rightarrow A^{n}(Y, Z) \stackrel{\delta^{n}}{\rightarrow} A^{n+1}(X, Y) \rightarrow \ldots
$$


Here the connecting morphism $\delta^{n}$ is a natural transformation of functors.

(4) Let $(Y \subseteq X)$ be a pair of topological spaces, and $U \subseteq X$ an open subset whose closure is contained in the interior of $Y$. Then the induced map

$$
A^{n}(X, Y) \rightarrow A^{n}(X-U, Y-U)
$$

is an isomorphism.

(5) If $X$ is a disjoint union of a collection of spaces $\left\{X_{\alpha}\right\}$, then for every integer $n$ the induced map $A^{n}(X) \rightarrow$ $\prod A^{n}\left(X_{\alpha}\right)$ is an isomorphism.

(6) If $X$ is a point, then

$$
A^{n}(X)= \begin{cases}0 & \text { if } n \neq 0 \\ \mathbf{Z} & \text { if } n=0\end{cases}
$$

Any collection of functors (and connecting maps $\delta^{n}$ ) satisfying the above axioms is necessarily isomorphic to the integral cohomology functors $(X \subseteq Y) \mapsto \mathrm{H}^{n}(X, Y ; \mathbf{Z})$. More generally, we can give a similar characterization of cohomology with coefficients in any abelian group $M$ : one simply replaces $\mathbf{Z}$ by $M$ in the statement of the dimension axiom (6).

A cohomology theory is a collection of functors $A^{n}$ (and connecting maps $\delta^{n}$ ) that satisfy the first five of the above axioms. Every abelian group $M$ gives rise to a cohomology theory, by setting $A^{n}(X, Y)=\mathrm{H}^{n}(X, Y ; M)$. But there are many other interesting examples; the study of these examples is the subject of stable homotopy theory.

If $A$ is a cohomology theory, we will write $A^{n}(X)$ for $A^{n}(X, \emptyset)$, and $A(X)$ for $A^{0}(X)$. Generally speaking, we will be interested in multiplicative cohomology theories: that is, cohomology theories for which each of the groups $A^{*}(X)$ is equipped with the structure of a graded commutative ring (graded commutativity means that $u v=(-1)^{n m} v u$ for $u \in A^{n}(X), v \in A^{m}(X)$; we will not take the trouble to spell out the complete definition).

Arguably the most interesting example of a cohomology theory, apart from ordinary cohomology, is complex $K$-theory. If $X$ is a reasonably nice space (for example, a finite cell complex), then $K(X)$ coincides with the Grothendieck group of stable isomorphism classes of complex vector bundles on $X$. Complex $K$ theory is a multiplicative cohomology theory, with multiplication determined by tensor products of complex vector bundles.

We observe that the dimension axiom fails dramatically for complex $K$-theory. Instead of being concentrated in degree zero, the graded ring $K^{*}(*) \simeq \mathbf{Z}\left[\beta, \beta^{-1}\right]$ is a ring of Laurent polynomials in a single indeterminate $\beta \in K^{-2}(*)$. Here $\beta$ is called the Bott element, because multiplication by $\beta$ induces isomorphisms

$$
K^{n}(X) \rightarrow K^{n-2}(X)
$$

for every space $X$ and every integer $n$ : this is the content of the famous Bott periodicity theorem (see [5]).

The following definition abstracts some of the pleasant properties of complex $K$-theory.

Definition 1.1. Let $A$ be a multiplicative cohomology theory. We will say that $A$ is even if $A^{i}(*)=0$ whenever $i$ is odd. We will say that $A$ is periodic if there exists an element $\beta \in A^{-2}(*)$ such that $\beta$ is invertible in $A^{*}(*)$ (so that $\beta$ has an inverse $\beta^{-1} \in A^{2}(*)$ ).

Complex $K$-theory is the prototypical example of an even periodic cohomology theory, but there are many other examples. Ordinary cohomology $\mathrm{H}^{*}(X ; R)$ with coefficients in a commutative ring $R$ is obviously even, but not periodic: in fact, we have $\mathrm{H}^{n}(* ; R)=0$ for $n \neq 0$. However, we can correct this problem by enforcing periodicity: namely, define

$$
A^{n}(X)=\prod_{k \in \mathbf{Z}} H^{n+2 k}(X ; R) .
$$

Then $A$ is an even periodic cohomology theory: we refer to this theory as periodic cohomology with coefficients in $R$. We will meet more exotic examples of even periodic cohomology theories in $\S 1.3$. 


\subsection{Formal Groups from Cohomology Theories}

Let $A$ be a cohomology theory, and $X$ a topological space. The Atiyah-Hirzebruch spectral sequence allows one to compute the $A$-cohomology groups of the space $X$ in terms of the $A$-cohomology groups of a point and the ordinary cohomology groups of the space $X$. More specifically, there is a spectral sequence with

$$
E_{2}^{p q}=H^{p}\left(X ; A^{q}(*)\right) \Rightarrow A^{p+q}(X) .
$$

In general, the Atiyah-Hirzebruch spectral sequence can be quite complicated. However, if $A$ is an even periodic cohomology theory, and $X$ is a space whose ordinary cohomology is concentrated in even degrees, then the situation simplifies drastically: the Atiyah-Hirzebruch spectral sequence degenerates at $E_{2}$, since there are no possible differentials for dimensional reasons. The most important example is the case in which $X$ is the infinite dimensional complex projective space $\mathbf{C P}^{\infty}$; in this case one can compute that

$$
A\left(\mathrm{CP}^{\infty}\right)
$$

is (noncanonically) isomorphic to a formal power series ring $A(*)[[t]]$ in one variable over the commutative ring $A(*)$.

There is an analogous computation for the ordinary cohomology ring of $\mathbf{C P}^{\infty}$ : we have

$$
\mathrm{H}^{*}\left(\mathbf{C P}^{\infty} ; \mathbf{Z}\right)=\mathbf{Z}[t],
$$

where $t \in \mathrm{H}^{2}\left(\mathbf{C P}^{\infty}, \mathbf{Z}\right)$ is the first Chern class of the universal line bundle $\mathcal{O}(1)$ on $\mathbf{C P}^{\infty}$. We have used the 2-periodicity of the cohomology theory $A$ to shift the generator from degree 2 to degree 0.

Remark 1.1. The reader might object that the analogy is imperfect, since the ordinary cohomology ring $\mathrm{H}^{*}\left(\mathbf{C P}{ }^{\infty}, \mathbf{Z}\right)$ is a polynomial ring, rather than a power series ring. However, this is dependent on our procedure for extracting an ordinary ring from a positively graded ring $\left\{R^{n}\right\}_{n>0}$. The usual convention is to define $R=\oplus_{n \geq 0} R^{n}$; however, one could instead consider the product $\prod_{n \geq 0} R^{n}$. The latter is more natural in the present context, because it can also be interpreted as the (degree zero) periodic cohomology of the space $\mathrm{CP}^{\infty}$, which is a power series ring rather than a polynomial ring.

By analogy with the case of ordinary cohomology, we may view the parameter $t$ in the isomorphism $A\left(\mathbf{C P}^{\infty}\right) \simeq A(*)[[t]]$ as the first Chern class of the universal line bundle $\mathcal{O}(1)$. In fact, once we fix a choice of the parameter $t$, we can use $t$ to define the first Chern class of any complex line bundle $\mathcal{L}$ on any space $X$. The space $\mathbf{C P}^{\infty}$ is a classifying space for complex line bundles: that is, for any complex line bundle $\mathcal{L}$ on a (paracompact) space $X$, there is a classifying map $\phi: X \rightarrow \mathbf{C P}^{\infty}$ and an isomorphism $\mathcal{L} \simeq \phi^{*} \mathcal{O}(1)$. The map $\phi$ is unique up to homotopy, so we may define

$$
c_{1}(\mathcal{L})=\phi^{*} t \in A(X) .
$$

This gives rise to a reasonably well-behaved theory of the first Chern class in $A$-cohomology. (By elaborating on this construction, one can construct a theory of higher Chern classes as well, but we will not need this.)

In ordinary cohomology, there is a simple formula that describes the first Chern class of a tensor product of two line bundles:

$$
c_{1}\left(\mathcal{L} \otimes \mathcal{L}^{\prime}\right)=c_{1}(\mathcal{L})+c_{1}\left(\mathcal{L}^{\prime}\right) .
$$

There is no reason to expect this formula to hold in general, and in fact it does not.

Example 1.1. In complex $K$-theory, there is an even simpler theory of Chern classes for line bundles. Namely, if $\mathcal{L}$ is a complex line bundle on a space $X$, then we may regard $\mathcal{L}$ itself as representing an element of $K(X)$; we will denote this element by $[\mathcal{L}]$. We now define $c_{1}(\mathcal{L})=[\mathcal{L}]-1$. (The reason for the subtraction is that we wish to normalize, so that the trivial line bundle has first Chern class equal to zero.) Now a simple computation shows that

$$
c_{1}\left(\mathcal{L} \otimes \mathcal{L}^{\prime}\right)=c_{1}(\mathcal{L})+c_{1}\left(\mathcal{L}^{\prime}\right)+c_{1}(\mathcal{L}) c_{1}\left(\mathcal{L}^{\prime}\right) .
$$


Returning to the general case, what we can assert is that there is always some formula which expresses $c_{1}\left(\mathcal{L} \otimes \mathcal{L}^{\prime}\right)$ in terms of $c_{1}(\mathcal{L})$ and $c_{1}\left(\mathcal{L}^{\prime}\right)$. To see this, it suffices to consider the universal example: that is, let $X$ be the classifying space $\mathbf{C P}^{\infty} \times \mathbf{C P}^{\infty}$ for pairs of complex line bundles. Like $\mathbf{C P}^{\infty}$, this is a space with only even-dimensional cohomology, so a relatively simple computation shows that

$$
A\left(\mathbf{C P}^{\infty} \times \mathbf{C P}^{\infty}\right) \simeq A(*)\left[\left[t_{1}, t_{2}\right]\right] .
$$

Here we can take $t_{1}=\pi_{1}^{*} t$ and $t_{2}=\pi_{2}^{*} t$, where $\pi_{1}$ and $\pi_{2}$ are the projections from the product $\mathbf{C P}^{\infty} \times \mathbf{C P}^{\infty}$ onto the first and second factor, respectively. Phrased another way, the power series generators $t_{1}$ and $t_{2}$ are the first Chern classes of the universal line bundles $\pi_{1}^{*} \mathcal{O}(1)$ and $\pi_{2}^{*} \mathcal{O}(1)$ on $\mathbf{C P}^{\infty} \times \mathbf{C P}^{\infty}$. We now observe that

$$
c_{1}\left(\pi_{1}^{*} \mathcal{O}(1) \otimes \pi_{2}^{*} \mathcal{O}(1)\right)=f\left(t_{1}, t_{2}\right) \in A(*)\left[\left[t_{1}, t_{2}\right]\right]
$$

for some (uniquely determined) power series $f$. Roughly speaking, we can assert that, by universality, we have $c_{1}\left(\mathcal{L} \otimes \mathcal{L}^{\prime}\right)=f\left(c_{1}(\mathcal{L}), c_{1}\left(\mathcal{L}^{\prime}\right)\right)$ for any pair of complex line bundles $\left(\mathcal{L}, \mathcal{L}^{\prime}\right)$ on any space $X$.

Remark 1.2. The above assertion is somewhat vague, since it is not clear how to evaluate a formal power series on a pair of elements in the commutative ring $A(X)$. However, if $X$ admits a finite cell decomposition, then for any line bundle $\mathcal{L}$ on $X$, the element $c_{1}(\mathcal{L}) \in A(X)$ is nilpotent (more specifically, $c_{1}(\mathcal{L})^{n}=0$ as soon as $\mathcal{L}$ is generated by $n$ sections). For such spaces $X$, it does make sense to evaluate the formal series $f\left(c_{1}(\mathcal{L}), c_{1}\left(\mathcal{L}^{\prime}\right)\right)$, and one obtains $c_{1}\left(\mathcal{L} \otimes \mathcal{L}^{\prime}\right)$.

The power series $f(u, v)$ is not arbitrary; it necessarily satisfies certain identities which reflect the properties of complex line bundles under multiplication:

(L1) Because the first Chern class of the trivial bundle is zero, we have the identities

$$
f(t, 0)=f(0, t)=t .
$$

(L2) Because the tensor product operation on complex line bundles is commutative up to isomorphism, we obtain the identity $f(u, v)=f(v, u)$.

(L3) Because the tensor product operation on complex line bundles is associative up to isomorphism,

$$
f(u, f(v, w))=f(f(u, v), w) .
$$

A power series $f(u, v)$ with the properties enumerated above is called a commutative, 1-dimensional formal group law over the commutative ring $A(*)$. Such a formal group law determines a group structure on the formal scheme $\operatorname{Spf} A(*)[[t]]=\operatorname{Spf} A\left(\mathbf{C P}^{\infty}\right)$.

Remark 1.3. The formal group law $f$ is not canonically associated to the cohomology theory $A$ : it depends on the choice of a power series generator $t$ for the ring $A\left(\mathbf{C P}^{\infty}\right) \simeq A(*)[[t]]$. However, the underlying formal group is independent of the choice of $t$ : namely, it is given by the formal spectrum $\operatorname{Spf} A\left(\mathbf{C P}^{\infty}\right)$. The group structure

$$
\operatorname{Spf} A\left(\mathbf{C P}^{\infty}\right) \times \operatorname{Spf} A\left(\mathbf{C P}^{\infty}\right) \rightarrow \operatorname{Spf} A\left(\mathbf{C P}^{\infty}\right)
$$

is induced by a map $s: \mathbf{C P}^{\infty} \times \mathbf{C P}^{\infty} \rightarrow \mathbf{C P}^{\infty}$ which classifies the operation of tensor product on complex line bundles. In more concrete terms, we can identify $\mathbf{C P}^{\infty}$ with the space of lines in the field of rational functions $\mathbf{C}(x)$ (viewed as an infinite dimensional complex vector space); the map $s$ is now induced by the multiplication map $\mathbf{C}(x) \otimes \mathbf{C}(x) \rightarrow \mathbf{C}(x)$.

Remark 1.4. Not every one-dimensional formal group $\mathbb{G}$ over a commutative ring $R$ arises from a formal group law: this requires the existence of a coordinate on the group $\mathbb{G}$, which in general exists only Zariski locally on $\operatorname{Spec} R$. Consequently, it is convenient to introduce a slightly more general notion of periodicity. We will say that a multiplicative cohomology theory $A$ is weakly periodic if the natural map 


$$
A^{2}(*) \otimes_{A(*)} A^{n}(*) \rightarrow A^{n+2}(*)
$$

is an isomorphism for all $n \in \mathbf{Z}$. Note that this implies in particular that $A^{2}(*) \otimes_{A(*)} A^{-2}(*) \simeq A(*)$, so that $A^{2}(*)$ is a projective module of rank 1 over $A(*)$. We note that $A$ is periodic if and only if it is weakly periodic and $A^{2}(*)$ is a free module over $A(*)$.

Example 1.2. The formal power series $f(u, v)=u+v$ determines a formal group over an arbitrary commutative ring $R$, which we call the formal additive group and denote by $\widehat{\mathbb{G}}_{a}$. As we have seen, this is the formal group which governs the behavior of Chern classes in (periodic) ordinary cohomology (with coefficients in $R)$.

Example 1.3. The formal power series $f(u, v)=u+v+u v$ determines a formal group over an arbitrary commutative ring $R$, which we call the formal multiplicative group and denote by $\widehat{\mathbb{G}}_{m}$. In the case where $R=\mathbf{Z}$, this is the formal group which governs the behavior of Chern classes in complex K-theory. The role of the multiplicative group is easy to understand in this case: modulo a normalization, we have essentially defined the first Chern class of a line bundle $\mathcal{L}$ to be the class $[\mathcal{L}]$ represented by $\mathcal{L}$ in K-theory. Tensor product of line bundles then correspond to products in K-theory.

It is natural to ask whether there are any restrictions on the power series $f(u, v)$, other than the identities $(L 1),(L 2)$, and (L3). To address this question, it is convenient to introduce a cohomology theory MP, called periodic complex cobordism. This cohomology theory is in some sense a universal home for the first Chern class of complex line bundles; in particular, there is a canonical isomorphism $\operatorname{MP}\left(\mathbf{C P}^{\infty}\right) \simeq \operatorname{MP}(*)[[t]]$. Quillen proved that the associated formal group law over $\mathrm{MP}(*)$ was also universal. In other words, the coefficient ring $\mathrm{MP}(*)$ is the Lazard ring which classifies formal group laws, so that for any commutative ring $R$ there is a bijection between the set $\operatorname{Hom}(\mathrm{MP}(*), R)$ of commutative ring homomorphisms from $\operatorname{MP}(*)$ into $R$, and the set of power series $f(u, v)$ with coefficients in $R$ that satisfy the three identities asserted above. We refer the reader to [1] for a proof of Quillen's theorem and further discussion.

The construction that associates the formal group $\mathbb{G}=\operatorname{Spf} A\left(\mathbf{C P}^{\infty}\right)$ to an even periodic cohomology theory $A$ has turned out to be a very powerful tool in stable homotopy theory. The reason is that the formal group $\mathbb{G}$ retains a remarkable amount of information about $A$. In many cases, it is possible to recover $A$ from the formal group $\mathbb{G}$. Indeed, suppose that $R$ is any commutative ring and $\mathbb{G}$ a formal group over $R$ determined by a formal group law $f(u, v) \in R[[u, v]]$. According to Quillen's theorem, this data is classified by a (uniquely determined) ring homomorphism $\mathrm{MP}(*) \rightarrow R$. There is a natural candidate for a cohomology theory $A_{\mathbb{G}}$ which gives rise to the formal group $\mathbb{G}$. Namely, for every finite cell complex $X$, set

$$
A_{\mathbb{G}}^{n}(X)=\mathrm{MP}^{n}(X) \otimes_{\mathrm{MP}(*)} R .
$$

Remark 1.5. To give a definition which does not involve finiteness restrictions on $X$, one should work with homology rather than cohomology; we will not concern ourselves with this technical point.

In general, the above formula for $A_{\mathbb{G}}$ does not give a cohomology theory. The problem is that long exact sequences in MP-cohomology might not remain exact after tensoring with $R$. If $R$ is flat over $\mathrm{MP}(*)$, then this problem disappears. However, a much weaker condition on $(R, \mathbb{G})$ will ensure the same conclusion. In order to formulate it, it is convenient to employ the language of algebraic stacks.

Let $\mathcal{M}_{\mathrm{FGL}}$ denote the moduli stack of formal group laws, so that for any commutative ring $R$ the set $\operatorname{Hom}\left(\operatorname{Spec} R, \mathcal{M}_{\mathrm{FGL}}\right)$ may be identified with the set of all power series $f(u, v) \in R[[u, v]]$ satisfying $(L 1)$, $(L 2)$, and $(L 3)$. Then $\mathcal{M}_{\mathrm{FGL}}$ is an affine scheme: in fact, by Quillen's theorem we have $\mathcal{M}_{\mathrm{FGL}}=\operatorname{Spec} \mathrm{MP}(*)$. Let $G$ denote the group scheme of all automorphisms of the formal affine line $\operatorname{Spf} \mathbf{Z}[[x]]$; in other words, $\operatorname{Hom}(\operatorname{Spec} R, G)$ is the set of all power series

$$
g(x)=a_{1} x+a_{2} x^{2}+\ldots
$$

with coefficients in $R$, such that $a_{1}$ is invertible; such power series form a group under composition. The group $G$ acts on $\mathcal{M}_{\mathrm{FGL}}$ : on the level of $R$-valued points, this action is given by the formula 


$$
f^{g}(u, v)=g^{-1} f(g(u), g(v))
$$

Let $\mathcal{M}_{\mathrm{FG}}$ denote the stack-theoretic quotient of $\mathcal{M}_{\mathrm{FGL}}$ by $G$ : this is the moduli stack of formal groups. There is a natural map

$$
\mathcal{M}_{\mathrm{FGL}} \rightarrow \mathcal{M}_{\mathrm{FG}}
$$

which "forgets the coordinate"; it is a principal bundle with structure group $G$.

Remark 1.6. One can think of $\mathcal{M}_{\mathrm{FG}}$ as a kind of infinite-dimensional Artin stack.

Now, for any space $X$, the MP-cohomology groups $\operatorname{MP}^{n}(X)$ are modules over the commutative ring $\mathrm{MP}(*)$. By Quillen's theorem, these modules can be identified with quasi-coherent sheaves on $\mathcal{M}_{\mathrm{FGL}}$. However, one can say more. If $A$ is a cohomology theory, a stable cohomology operation (of degree $\mathrm{k}$ ) is a collection of natural transformations

$$
A^{n}(X, Y) \rightarrow A^{n+k}(X, Y)
$$

which are suitably compatible with the connecting homomorphism. For example, if $A$ is a multiplicative cohomology theory, then every element of $u \in A^{k}(*)$ gives rise to a stable cohomology operation, given by multiplication by $u$. However, in the case where $A=\mathrm{MP}$, there are many other stable cohomology operations. For any space $X$, the cobordism group $\mathrm{MP}(X)$ is a module over the ring of all stable cohomology operations on MP. An elaboration of Quillen's theorem can be used to give an algebro-geometric interpretation of this additional structure: when $X$ is finite cell complex, the quasi-coherent sheaves $\operatorname{MP}^{n}(X)$ on $\mathcal{M}_{\mathrm{FGL}}$ are endowed with an action of the group $G$ (covering the action of $G$ on $\mathcal{M}_{\mathrm{FGL}}$ ). In other words, we may interpret the complex cobordism groups $\operatorname{MP}^{n}(X)$ as quasi-coherent sheaves $\mathcal{M} \mathcal{P}^{n}(X)$ on the moduli stack $\mathcal{M}_{\mathrm{FG}}$.

Remark 1.7. The finiteness restrictions on $X$ in the preceding discussion can be dropped if we are willing to work with the MP-homology groups of $X$, rather than the MP-cohomology groups.

Now suppose that $\mathbb{G}$ is a (commutative, 1-dimensional) formal group over a commutative ring $R$. Then $\mathbb{G}$ is classified by a map

$$
\operatorname{Spec} R \stackrel{\phi}{\rightarrow} \mathcal{M}_{\mathrm{FG}}
$$

and we may define

$$
A^{n}(X)=\phi^{*} \mathcal{M} \mathcal{P}^{n}(X)
$$

for every finite cell complex $X$; this is a quasi-coherent sheaf on $\operatorname{Spec} R$ which we may identify with an $R$-module. Again, this does not necessarily define a cohomology theory: however, it does give a cohomology theory whenever the map $\phi$ is flat. If $\phi$ is flat, then we say that the formal group $\mathbb{G}$ is Landweber-exact. Using the structure theory of formal groups, Landweber has given a criterion for a formal group $\mathbb{G}$ to be Landweber-exact (see [19]). We will not review Landweber's theorem here. However, we remark that Landweber's criterion is purely algebraic, easy to check, and is quite often satisfied.

Remark 1.8. In the case where $\mathbb{G}$ is the formal group given by a formal group law $f(u, v) \in R[[u, v]]$, the preceding definition of $A^{n}(X)$ coincides with the definition given earlier. In general, the formal group law $f$ exists only (Zariski) locally on $\operatorname{Spec} R$; namely, it exists whenever the Lie algebra $\mathfrak{g}$ of $\mathbb{G}$ is free as an $R$ module. The present definition of $A$ has the advantage that it does not depend on a choice of coordinate on the formal group $\mathbb{G}$, and makes sense even when the Lie algebra $\mathfrak{g}$ is not free. Note that if $\mathfrak{g}$ is not free, then $A$ cannot be a periodic cohomology theory in the sense of Definition 1.1: instead, we have $A^{2 k}(*) \simeq \mathfrak{g}^{\otimes(-k)}$, so that $A$ is weakly periodic in the sense of Remark 1.4.

Example 1.4. Let $R=\mathbf{Z}$, and let $\widehat{\mathbb{G}}_{m}$ be the formal multiplicative group (determined by the formal group law $f(u, v)=u+v+u v)$. In this case, Landweber's criterion is satisfied, so that

$$
A^{n}(X)=\mathrm{MP}^{n}(X) \otimes_{\mathrm{MP}(*)} \mathbf{Z}
$$


defines a cohomology theory (for finite cell complexes $X$ ). Moreover, this cohomology theory is uniquely determined by the fact that it is even, periodic, and is associated to the formal multiplicative group $\widehat{\mathbb{G}}_{m}$ over $A(*) \simeq \mathbf{Z}$. We saw in Example 1.3 that complex $K$-theory has all of these properties. We deduce that $A$ is complex $K$-theory: in other words, we can discover $K$-theory in a purely algebraic fashion, by thinking about the formal multiplicative group over the integers.

Example 1.5. Let $\widehat{\mathbb{G}}_{a}$ be the formal additive group over a commutative ring $R$, defined by the formal group law $f(u, v)=u+v$. Then $\widehat{\mathbb{G}}_{a}$ is Landweber-exact if and only if $R$ is an algebra over the field $\mathbf{Q}$ of rational numbers. In this case, the associated cohomology theory is just periodic cohomology with coefficients in $R$.

Remark 1.9. Over the field $\mathbf{Q}$ of rational numbers, the formal additive group is isomorphic to the formal multiplicative group (in fact, all commutative, 1-dimensional formal groups over $\mathbf{Q}$ are isomorphic to one another). This reflects the fact that rationally, complex K-theory reduces to periodic cohomology with rational coefficients. More precisely, for every finite cell complex $X$, the Chern character gives an isomorphism

$$
\operatorname{ch}: K(X) \otimes \mathbf{z} \mathbf{Q} \rightarrow \prod_{k} \mathrm{H}^{2 k}(X ; \mathbf{Q}) .
$$

The Chern character should be thought of as a cohomological reflection of the isomorphism exp : $\widehat{\mathbb{G}}_{a} \stackrel{\sim}{\rightarrow} \widehat{\mathbb{G}}_{m}$.

\subsection{Elliptic Cohomology}

In the last section, we reviewed the relationship between cohomology theories and formal groups. In particular, we saw that the most basic examples of formal groups (namely, the formal additive group $\widehat{\mathbb{G}}_{a}$ and the formal multiplicative group $\widehat{\mathbb{G}}_{m}$ ) were closely related to the most basic examples of cohomology theories (namely, ordinary cohomology and complex K-theory). It is natural to try to expand on these examples, by seeking out other cohomology theories that are related to interesting formal groups.

A key feature of the formal groups $\widehat{\mathbb{G}}_{a}$ and $\widehat{\mathbb{G}}_{m}$ is that they arise as the formal completions of the algebraic groups $\mathbb{G}_{a}$ and $\mathbb{G}_{m}$. (The homotopy-theoretic significance of this observation will become clear later, when we discuss equivariant cohomology theories.) Since we are interested only in commutative, 1-dimensional formal groups, it is natural to consider algebraic groups which are also commutative and 1-dimensional. However, examples are in short supply: over an algebraically closed field, every 1-dimensional algebraic group is isomorphic to either the additive group $\mathbb{G}_{a}$, the multiplicative group $\mathbb{G}_{m}$, or an elliptic curve. The cohomology theories associated to the first two examples are classical, but the third case is more exotic: this is the subject of elliptic cohomology, to which this paper is devoted.

Definition 1.2. An elliptic cohomology theory consists of the following data:

(1) A commutative ring $R$.

(2) An elliptic curve E, defined over $R$.

(3) A multiplicative cohomology theory $A$ which is even and weakly periodic (see Remark 1.4).

(4) Isomorphisms $A(*) \simeq R$ and $\widehat{E} \simeq \operatorname{Spf} A\left(\mathbf{C P}^{\infty}\right)$ (of formal groups over $R \simeq A(*)$ ). Here $\widehat{E}$ denotes the formal completion of the elliptic curve $E$ along its identity section.

Remark 1.10. In the situation of Definition 1.2, we will often abuse terminology and simply refer to $A$ as an elliptic cohomology theory. In other words, we think of an elliptic cohomology theory as a cohomology theory $A$, together with some additional data relating $A$ to an elliptic curve.

Remark 1.11. Let $E$ be an elliptic curve over a commutative ring $R$. The formal completion $\widehat{E}$ of $E$ is a commutative, 1-dimensional formal group over $R$. If $\widehat{E}$ is Landweber-exact, then the data of (3) and (4) is uniquely determined. Consequently, in the Landweber-exact case, an elliptic cohomology theory is essentially the same thing as an elliptic curve. 
Remark 1.12. Our notion of elliptic cohomology theory is essentially the same as the notion of an elliptic spectrum as defined in defined in [4].

In view of Remark 1.11, there is a plentiful supply of elliptic cohomology theories: roughly speaking, there is an elliptic cohomology theory for every elliptic curve. Of course, we have a similar situation for other algebraic groups. The multiplicative group $\mathbb{G}_{m}$ can be defined over any commutative ring $R$. The formal completion of $\mathbb{G}_{m}$ is Landweber-exact if and only if $R$ is a torsion-free $\mathbf{Z}$-module; in this case, we have an associated cohomology theory which is given by

$$
A^{n}(X)=K^{n}(X) \otimes \mathbf{z} R
$$

for every finite cell complex $X$. In other words, there are many cohomology theories associated to the multiplicative group, but they are all just variants of one universal example: complex $K$-theory, associated to the "universal" multiplicative group over the ring of integers $\mathbf{Z}$.

The analogous situation for elliptic cohomology is more complicated, because there is no universal example of an elliptic curve over a commutative ring. In other words, the moduli stack $\mathcal{M}_{1,1}$ of elliptic curves, defined by the equation

$$
\operatorname{Hom}\left(\operatorname{Spec} R, \mathcal{M}_{1,1}\right)=\{\text { Elliptic curves } E \rightarrow \operatorname{Spec} R\},
$$

is not an affine scheme. In fact, $\mathcal{M}_{1,1}$ is not even a scheme: the right hand side of the above equation really needs to be viewed as a groupoid, since elliptic curves can have nontrivial automorphisms. However, $\mathcal{M}_{1,1}$ is a Deligne-Mumford stack; that is, there is a sufficient supply of étale morphisms $\phi: \operatorname{Spec} R \rightarrow \mathcal{M}_{1,1}$. For every such morphism $\phi$ there is associated an elliptic curve $E_{\phi}$. It turns out that, if $\phi$ is étale (or more generally, if $\phi$ is flat), then the formal group $\widehat{E}_{\phi}$ is Landweber-exact. Consequently, in this case we may associate to $\phi$ an elliptic cohomology theory $A_{\phi}$.

The correspondence

$$
\overline{\mathcal{O}}:\left[\phi: \operatorname{Spec} R \rightarrow \mathcal{M}_{1,1}\right] \mapsto A_{\phi}
$$

determines a presheaf on $\mathcal{M}_{1,1}$, taking values in the category of cohomology theories. (More precisely, it is a presheaf of cohomology theories on the category of affine schemes with an étale map to $\mathcal{M}_{1,1}$.) This presheaf may, in some sense, be regarded as the "universal elliptic cohomology theory". To extract an actual cohomology theory from $A$, it is tempting to try to extract some sort of global sections $\Gamma\left(\mathcal{M}_{1,1}, \overline{\mathcal{O}}\right)$. Unfortunately, the notion of a cohomology theory is not well-suited to this sort of construction: one cannot generally make sense of the global sections of a presheaf that takes values in the category of cohomology theories.

To remedy this difficulty, it is necessary to represent our cohomology theories. According to Brown's representability theorem (see [2]), any cohomology theory $A$ has a representing space $Z$, so that there is a functorial identification

$$
A(X) \simeq[X, Z]
$$

of the $A$-cohomology of every cell complex $X$ with the set $[X, Z]$ of homotopy classes of maps from $X$ into $Z$. More generally, for each $n \in \mathbf{Z}$ there is a space $Z(n)$ and an identification

$$
A^{n}(X) \simeq[X, Z(n)] .
$$

The connecting map in the $A$-cohomology long exact sequence endows the sequence of spaces $\{Z(n)\}$ with additional structure: namely, a sequence of homotopy equivalences

$$
\delta(n): Z(n) \rightarrow \Omega Z(n+1) .
$$

A sequence of (pointed) spaces $Z(n)$, together with homotopy equivalences $\delta(n)$ as above, is called a spectrum. Every spectrum determines a cohomology theory, and every cohomology theory arises in this way. However, the spectrum representing a cohomology theory $A$ is not canonically determined by $A$ : for example, the 0 th space $Z(0)$ can only be recovered up to homotopy equivalence. 
Consider the diagram

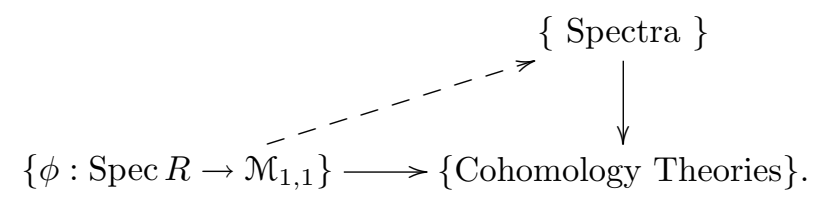

If we could supply the dotted arrow, then we could lift the presheaf $\overline{\mathcal{O}}$ to a presheaf of spectra on the moduli stack of elliptic curves. This would address the problem: there is a good theory of sheaves of spectra, which would allow us to form a spectrum of global sections. However, supplying the dotted arrow is no simple matter. It is an example of a lifting problem which, in principle, can be attacked using the methods of obstruction theory. However, the obstruction-theoretic calculations involved turn out to be very difficult, so that a direct approach is not feasible.

The fundamental insight, due to Hopkins and Miller, is that the requisite calculations are much more tractable if we try to prove a stronger result. By definition, an elliptic cohomology theory is required to have a multiplicative structure. If $A$ is a multiplicative cohomology theory represented by a spectrum $\{Z(n)\}$, then by Yoneda's lemma, the multiplication map

$$
A^{k}(X) \times A^{l}(X) \rightarrow A^{k+l}(X)
$$

is represented by a map $m_{k, l}: Z(k) \times Z(l) \rightarrow Z(k+l)$, which is well-defined up to homotopy. Moreover, the identities that are satisfied by the multiplication operation may be rephrased in terms of certain diagrams involving the maps $m_{k, l}$, which are required to commute up to homotopy. In particular, the space $Z(0)$ has the structure of a commutative ring, when regarded as an object in the homotopy category of topological spaces. For sophisticated purposes, requiring the ring axioms to hold up to homotopy is not nearly good enough. However, one does not wish to require too much. We could ask that the ring axioms for $Z(0)$ hold not just up to homotopy, but on the nose, so that $Z(0)$ is a topological commutative ring. Any topological commutative ring represents a multiplicative cohomology theory. However, it turns out that the cohomology theories which arise in this way are not very interesting: they are all just variants of ordinary cohomology. In particular, the classifying space $\mathbf{Z} \times B U$ for complex $K$-theory is not homotopy equivalent to a topological commutative ring.

There is a notion that is intermediate between "commutative ring up to homotopy" and "topological commutative ring", which is suitable for describing the kind of multiplicative structure that exists on complex $K$-theory and in many other examples. Roughly speaking, one requires the representing space $Z(0)$ to have the structure of a commutative ring in the homotopy category of topological spaces, but also remembers the homotopies which make the relevant diagrams commute, which are required to satisfy further identities (also up to homotopies which are part of the structure and required to satisfy yet higher identites, and so on). A spectrum $\{Z(n)\}$ together with all of this data is called an $E_{\infty}$-ring spectrum, or simply an $E_{\infty}-$ ring. We will not give a definition here, though we will give a brief outline of the theory in $\S 2.1$. For definitions and further details we refer the reader to the literature (for example [12] or [17]).

Returning to the subject of elliptic cohomology, we can now consider the diagram

$$
\left.\left\{\phi: \operatorname{Spec} R \rightarrow \mathcal{M}_{1,1}^{-}\right\} \longrightarrow \text { Multiplicative Cohomology Theories }\right\} .
$$

Once again, our objective is to produce the dotted arrow in the diagram: as for spectra, there is a suitable sheaf theory for $E_{\infty}$-rings. At first glance, this appears to be a much more difficult problem than the one considered earlier. Let $\phi: \operatorname{Spec} R \rightarrow \mathcal{M}_{1,1}$ be an étale map, and let $A_{\phi}$ be the associated elliptic cohomology theory. The Brown representability theorem guarantees that $A_{\phi}$ can be represented by a spectrum. However, 
the multiplicative structure on $A_{\phi}$ does not guarantee us an $E_{\infty}$-structure on the representing spectrum. Thus, the problem of lifting $\overline{\mathcal{O}}$ to a sheaf of $E_{\infty}$-rings is nontrivial, even when we restrict $\overline{\mathcal{O}}$ to a single object.

However, it turns out that problem of lifting $\overline{\mathcal{O}}$ to a presheaf of $E_{\infty}$-rings is much more amenable to obstruction-theoretic attack. This is because $E_{\infty}$-rings are very rigid objects. Although it is much harder to write down an $E_{\infty}$-ring than a spectrum, it is also much harder to write down a map between $E_{\infty}$-rings than a map between spectra. The practical effect of this, in our situation, is that it is much harder to write down the wrong maps between $E_{\infty}$-rings and much easier to find the right ones. Using this idea, Goerss, Hopkins and Miller were able to prove the following result

Theorem 1.1. There exists a commutative diagram

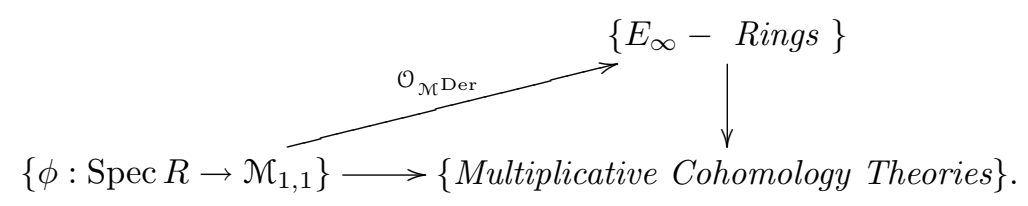

Moreover, the functor $\mathcal{O}_{\mathcal{M}^{D e r}}$ is determined uniquely up to homotopy equivalence.

We are now in a position to extract a "universal" elliptic cohomology theory, by taking the global sections of the presheaf $\mathcal{O}_{\mathcal{M}}$ Der . In the language of homotopy theory, this amounts to taking the homotopy limit of the functor $\mathcal{O}_{\mathcal{M}^{\text {Der }}}$. We will denote this homotopy limit by $\operatorname{tmf}\left[\Delta^{-1}\right]$. This is an $E_{\infty}$-ring: in other words, a cohomology theory with a very sophisticated multiplicative structure. In particular, we can view $\operatorname{tmf}\left[\Delta^{-1}\right]$ as a multiplicative cohomology theory. However, it is not an elliptic cohomology theory: in fact, it is neither even nor periodic (at least not of period 2). This is a reflection of the fact that the moduli stack $\mathcal{M}_{1,1}$ is not affine. In other words, $\operatorname{tmf}\left[\Delta^{-1}\right]$ is not an elliptic cohomology theory because it does not correspond to any particular elliptic curve: rather, it corresponds to all elliptic curves at once.

For our purposes, it is the (pre)sheaf $\mathcal{O}_{\mathcal{M}^{\text {Der }}}$ itself which is the principal object of interest, not the $E_{\infty}$-ring $\operatorname{tmf}\left[\Delta^{-1}\right]$ of global sections. Passage to global sections loses a great deal of interesting information, since the moduli stack $\mathcal{M}_{1,1}$ is not affine. In the next section, we will "rediscover" $\mathcal{O}_{\mathcal{M}^{\text {Der }}}$ from a rather different point of view: namely, by thinking about equivariant cohomology theories.

Remark 1.13. The reason that we have written $\operatorname{tmf}\left[\Delta^{-1}\right]$, rather than tmf, is that we considered above the moduli stack $\mathcal{M}_{1,1}$ of (smooth) elliptic curves. The cohomology theory tmf is associated to a similar picture, where we replace $\mathcal{M}_{1,1}$ by its Deligne-Mumford compactification $\overline{\mathcal{M}_{1,1}}$. We will discuss elliptic cohomology over $\overline{\mathcal{M}_{1,1}}$ in $\S 4.3$.

The notation tmf is an acronym for topological modular forms. It is so-named because there exists a ring homomorphism from $\operatorname{tmf}^{*}(*)$ to the ring of integral modular forms. This homomorphism is an isomorphism after inverting the primes 2 and 3. (These primes are troublesome because of the existence of elliptic curves with automorphisms of orders 2 and 3.$)$

\section{Derived Algebraic Geometry}

Many of the cohomology theories which appear "in nature" can be extended to equivariant cohomology theories. For example, if $X$ is a reasonably nice (compact) space with an action of a compact Lie group $G$, one defines the $G$-equivariant $K$-theory of $X$ to be the Grothendieck group $K_{G}(X)$ of $G$-equivariant vector bundles on $X$. We would like to consider the following general question: given a cohomology theory $A$, where should we look for $G$-equivariant versions of $A$-cohomology?

For any reasonable theory of equivariant cohomology, we expect that if $X$ is a principal $G$-bundle over a space $Y$, then there is a natural isomorphism $A_{G}(X) \simeq A(Y)$. This gives a definition of $A_{G}(X)$ whenever the action of $G$ on $X$ is sufficiently "free". However, one can always replace $X$ by a homotopy equivalent space with a free $G$-action. Namely, let $p: E G \rightarrow B G$ be a principal $G$-bundle whose total space $E G$ is 
contractible. Such a principal bundle always exists, and is uniquely determined up to homotopy equivalence. Now any space $X$ on which $G$ acts can be replaced by the homotopy equivalent space $E G \times X$, which is a principal $G$-bundle. One can now define $A_{G}^{\mathrm{Bor}}(X)=A((X \times E G) / G)$ : this is called the Borel-equivariant cohomology theory associated to $A$.

There are some respects in which Borel-equivariant cohomology is not a satisfying answer to our question. For one thing, we note that $A_{G}^{\text {Bor }}(X)$ is entirely determined by the original cohomology theory $A$ : in other words, it is nothing but a new notation for ordinary $A$-cohomology. More importantly, there are many cases in which it is not the answer that we want to get. For example, if $A$ is complex $K$-theory, then the Borelequivariant theory does not coincide with theory obtained by considering equivariant vector bundles. Instead, we have a natural map

$$
K_{G}(X) \rightarrow K_{G}(X \times E G) \simeq K((X \times E G) / G) \simeq K_{G}^{\mathrm{Bor}}(X)
$$

where the first map is given by pullback along the projection $X \times E G \rightarrow X$. This map is generally not an isomorphism. When $X$ is a point, we obtain the map

$$
\phi: \operatorname{Rep}(G) \rightarrow K(B G)
$$

from the representation ring of $G$ to the $K$-theory of $B G$, which carries each representation of $G$ to the associated bundle on the classifying space. This map is never an isomorphism unless $G$ is trivial. However, it is not far from being an isomorphism: by the Atiyah-Segal completion theorem ([6]), $\phi$ identifies $K(B G)$ with the completion of $\operatorname{Rep}(G)$ with respect to a certain ideal (the ideal consisting of virtual representations of dimension zero).

Let us consider the situation in more detail for the circle group $G=S^{1}$. Every representation of $G$ is a direct sum of irreducible representations. Since $G$ is abelian, every irreducible representation is one dimensional, given by a character $G \rightarrow \mathbf{C}^{*}$. Furthermore, every character of $G$ is simply an integral power of the "defining" character

$$
\chi: G \simeq\{z \in \mathbf{C}:|z|=1\} \hookrightarrow \mathbf{C}^{*} .
$$

The representation ring of $G$ is therefore isomorphic with the ring of Laurent polynomials $\mathbf{Z}\left[\chi, \chi^{-1}\right]$. We observe that this ring of Laurent polynomials may also be identified with the ring of functions on the multiplicative group $\mathbb{G}_{m}$. The classifying space of the circle group may be identified with $\mathbf{C P}^{\infty}$; as we saw in $\S 1.2$, the $K$-theory of this space is a power series ring $\mathbf{Z}[[t]]$, which may be identified with the ring of functions on the formal multiplicative group $\widehat{\mathbb{G}}_{m}$. In this case, the map

$$
K_{G}(*) \rightarrow K(B G)
$$

is easily identified: it is given by restriction of functions from the entire multiplicative group to the formal multiplicative group. In other words, it is given by taking germs of functions near the identity. Concretely, this homomorphism is described by the formula $\chi \mapsto(t+1)$.

We saw in $\S 1$ that if $A$ is an even periodic cohomology theory, then $A$ determines a formal group $\widehat{\mathbb{G}}=\operatorname{Spf} A\left(\mathbf{C P}^{\infty}\right)$. The lesson to learn from the example of $K$-theory is that the problem of finding equivariant versions of the cohomology theory $A$ may be related to the problem of realizing $\widehat{\mathbb{G}}$ as the formal completion of an algebraic group $\mathbb{G}$. In the case of $K$-theory, we can recover $\mathbb{G}$ as the spectrum of the equivariant $K$-group $K_{S^{1}}(*)$. In the case of elliptic cohomology, we do not expect $\mathbb{G}$ to be affine, and therefore we cannot expect to recover it from its ring of functions (though it is often possible to reconstruct $\mathbb{G}$ through a more elaborate procedure: we will discuss this problem in $§ 5.5)$. Instead, we should view the correspondence as running in the other direction: given an algebraic group $\mathbb{G}$ having $\operatorname{Spf} A\left(\mathbf{C P}^{\infty}\right)$ as its formal completion, it is natural to define $A_{S^{1}}(*)$ to be global sections of the structure sheaf of $\mathbb{G}$. Passing from functions to their formal germs then gives a suitable "completion" map

$$
A_{S^{1}}(*) \rightarrow A\left(\mathbf{C P}^{\infty}\right)
$$

from equivariant $A$-cohomology to Borel-equivarient $A$-cohomology. 
Of course, the above prescription does not solve the problem of defining equivariant versions of the cohomology theory $A$, even for the group $G=S^{1}$. Merely knowing the equivariant cohomology groups $A_{G}(*)$ of a point does not tell us how to define the equivariant cohomology groups $A_{G}(X)$ of a more general $G$-space $X$. This difficulty arises even when $G$ is trivial: a cohomology theory $A$ is not determined by its coefficient groups $A^{n}(*)$. To really exploit the ideas sketched above, we need to be able to extract from the algebraic group $\mathbb{G}$ not just cohomology rings, but cohomology theories. To accomplish this, we need $\mathbb{G}$ to be an algebraic group in a somewhat nonstandard setting: the sheaf of regular functions on $\mathbb{G}$ will be a sheaf not of ordinary commutative rings, but of $E_{\infty}$-rings. Our goal in $\S 2$ is to introduce the ideas which are needed to make sense of these kinds of algebraic groups. We will use this theory to construct equivariant cohomology theories in $\S 3$, and relate it to the theory of elliptic cohomology in $\S 4$.

\section{$2.1 E_{\infty}$-Rings}

In $\S 1.3$, we briefly mentioned the notion of an $E_{\infty}$-ring spectrum, which reappear throughout this paper. However, the ideas involved are somewhat nonelementary, and to give a precise definition would take us too far afield. This section is intended as a nontechnical introduction to the subject of $E_{\infty}$-ring theory; we will not give any definitions or prove any theorems, but will highlight some of the main features of the theory and explain how it can be used in practice.

Roughly speaking, an $E_{\infty}$-ring space is a topological space $A$ equipped with the structure of a commutative ring. As we explained in $\S 1.3$, there are several unsatisfactory ways of making this precise. Consider the diagram

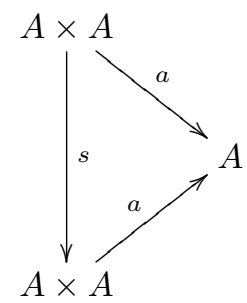

where $a$ denotes the addition map on $A$, and $s$ the automorphism of $A \times A$ which permutes the factors. The commutativity of this diagram expresses the commutativity of the addition operation on $A$. However, this is a very strong condition which is often not satisfied in practice. What is much more common is that the diagram commutes up to homotopy. That means that there is a continuous map

$$
h_{t}: A \times A \times[0,1] \rightarrow A
$$

with $h_{0}=a$ and $h_{1}=a \circ s$. For sophisticated applications, merely knowing the existence of $h$ is usually not enough: one should really take $h$ to be part of the ring structure on $A$. Moreover, $h$ should not be arbitrary, but should itself satisfy certain identities (at least up to homotopy, which must again be specified). In other words, we do not want $A$ merely to have the structure of a commutative ring up to homotopy, but up to coherent homotopy. Of course, deciding exactly what we mean by this is a highly nontrivial matter: exactly what homotopies should we take as part of the data, and what identities should they satisfy? There are several (equivalent) ways of answering these questions; we refer the reader to [12] for one possibility.

Let $A$ be an $E_{\infty}$-ring space; then, in particular, we can view $A$ as a space and consider its homotopy groups $\pi_{n} A$ (here we use a canonical base point of $A$ which is given by the "identity" with respect to addition). Since $A$ is an abelian group in the homotopy category of topological spaces, each $\pi_{n} A$ is endowed with the structure of an abelian group; this agrees with the usual group structure on $\pi_{n} A$ for $n \geq 1$. In addition, the multiplication operation on $A$ endows $\pi_{*} A=\oplus_{n \geq 0} \pi_{n} A$ with the structure of a graded ring. The ring $\pi_{*} A$ is commutative in the graded sense: that is, if $x \in \pi_{n} A$ and $y \in \pi_{m} B$, then $x y=(-1)^{n m} y x \in \pi_{n+m} A$. In particular, $\pi_{0} A$ is a commutative ring, and each $\pi_{n} A$ has the structure of a module over $\pi_{0} A$.

Any commutative ring can be regarded as an $E_{\infty}$-ring space; we simply regard it as a topological commutative ring, given the discrete topology. For any $E_{\infty}$-ring space $A$, there is a canonical map $A \rightarrow \pi_{0} A$ which collapses each path component of $A$ to a point. 
A map of $E_{\infty}$-ring spaces $A \rightarrow B$ is an equivalence if it gives rise to isomorphisms $\pi_{n} A \rightarrow \pi_{n} B$. We note that if $A$ is an $E_{\infty}$-ring space with $\pi_{n} A=0$ for $n \geq 1$, then the projection $A \rightarrow \pi_{0} A$ is an equivalence; in this case we say that $A$ is essentially discrete and we may abuse terminology by identifying $A$ with the ordinary commutative ring $\pi_{0} A$. We can regard the higher homotopy groups $\pi_{n} A$ as a measure of the difference between $A$ and the ordinary commutative ring $\pi_{0} A$.

Remark 2.1. Recall that an ordinary commutative ring $R$ is said to be reduced if it contains no nonzero nilpotent elements. If $R$ is not reduced, then the nilpotent elements of $R$ form an ideal $I$; then there is a projection $R \rightarrow R / I$, and $R / I$ is a reduced ring. The relationship between $E_{\infty}$-ring spaces and ordinary commutative rings should be regarded as analogous to the relationship between ordinary commutative rings and reduced commutative rings. If $A$ is an $E_{\infty}$-ring space, one should regard $\pi_{0} A$ as the "underlying ordinary commutative ring" which is obtained from $A$ by killing the higher homotopy groups, just as the reduced ring $R / I$ is obtained by killing the nilpotent elements of $R$.

Any $E_{\infty}$-ring space $A$ determines a cohomology theory: for a (well-behaved) topological space $X$, one can define $A(X)$ to be the set of homotopy classes of maps from $X$ into $A$. More generally, one can consider the space $A^{X}$ of all maps from $X$ into $A$, and endow it with the structure of an $E_{\infty}$-ring space, computing all of the ring operations pointwise. One can then define $A^{-n}(X)$ to be the homotopy group $\pi_{n} A^{X}$ for $n \geq 0$. This definition has a natural extension to the case $n<0$, and gives rise to a (multiplicative) cohomology theory which we will also denote by $A$. Of course, the functor

$$
X \mapsto A(X)
$$

determines $A$ as a topological space, up to weak homotopy equivalence. The $E_{\infty}$-ring structure on $A$ can be regarded as determining a commutative ring structure on the functor $X \mapsto A(X)$, together with certain higher coherence conditions.

We therefore have two distinct (but related) points of view on what an $E_{\infty}$-ring space $A$ is. On the one hand, we may view $A$ as a "commutative ring in homotopy theory"; from this point of view, the theory of $E_{\infty}$-rings is a kind of generalized commutative algebra. On the other hand, we may view an $E_{\infty}$-ring space $A$ as a cohomology theory equipped with a good multiplicative structure, giving rise not only to multiplication maps on $A$-cohomology but also secondary operations such as Massey products and their higher analogues. Both of these points of view will be important for the applications we discuss in this paper.

The cohomology theory associated to an $E_{\infty}$-ring space $A$ is connective: that is, it has the property that $A^{n}(*)=0$ for $n>0$. Many cohomology theories which arise naturally do not have this property. For example, a nontrivial cohomology theory cannot be both connective and periodic (in the sense of Definition 1.1). Consequently, it is necessary to introduce a slightly more general notion than an $E_{\infty}$-ring space: that of an $E_{\infty}$-ring spectrum, or simply an $E_{\infty}$-ring. Roughly speaking, an $E_{\infty}$-ring is a cohomology theory $A$ with all of the same good multiplicative properties that the cohomology theories associated to $E_{\infty}$-ring spaces have, but without the requirement that $A$ be connective. If $A$ is an $E_{\infty}$-ring, then one can associate to it a graded ring $\oplus_{n \in \mathbf{Z}} \pi_{n} A$ (by taking that $A$-cohomology groups of a point) which may be nonzero in both positive and negative degrees. Every $E_{\infty}$-ring space may be regarded as an $E_{\infty}$-ring; conversely, an $E_{\infty}$-ring $A$ with $\pi_{n} A=0$ for $n<0$ is equivalent to an $E_{\infty}$-ring space. Finally, if $A$ is an arbitrary $E_{\infty}$-ring, then it has a connective cover $\tau_{\geq 0} A$, which satisfies

$$
\pi_{k}\left(\tau_{\geq 0} A\right)= \begin{cases}\pi_{k} A & \text { if } k \geq 0 \\ 0 & \text { if } k<0\end{cases}
$$

Remark 2.2. It is important to understand that the world of $E_{\infty}$-rings is essentially higher-categorical in nature. In practice, this means that given two $E_{\infty}$-rings $A$ and $B$, one really has a space $\operatorname{Hom}(A, B)$ of maps from $A$ to $B$, rather than simply a set.

If $A$ is an $E_{\infty}$-ring, then there is a good theory of modules over $A$, which are called $A$-module spectra. Every $A$-module spectrum $M$ can be viewed as a spectrum, and therefore determines a cohomology theory 
$X \mapsto M^{*}(X)$. In particular, one can define homotopy groups $\pi_{n} M=M^{-n}(*)$, and these form a graded module $\pi_{*} M$ over the graded ring $\pi_{*} A$.

We will generally refer to $A$-module spectra simply as $A$-modules. However, there is a special case in which this could potentially lead to confusion. If $A$ is an ordinary commutative ring, then we may regard $A$ as an $E_{\infty}$-ring. In this case, we can identify $A$-module spectra with objects of the derived category of $A$-modules: that is, chain complexes of $A$-modules, defined up to quasi-isomorphism.

The following definition will play an important role throughout this paper:

Definition 2.1. Let $A$ be an $E_{\infty}$-ring and let $M$ be an $A$-module. We will say that $M$ is flat if the following conditions are satisfied:

(1) The module $\pi_{0} M$ is flat over $\pi_{0} A$, in the sense of classical commutative algebra.

(2) For each $n$, the induced map

$$
\pi_{n} A \otimes_{\pi_{0} A} \pi_{0} M \rightarrow \pi_{n} M
$$

is an isomorphism.

We will say that a map $A \rightarrow B$ of $E_{\infty}$-rings is flat if $B$ is flat when regarded as an $A$-module.

\subsection{Derived Schemes}

In the last section, we reviewed the theory of $E_{\infty}$-rings, and saw that it was a natural generalization of classical commutative algebra. In this section, we will explain how to incorporate these ideas into the foundations of algebraic geometry.

We begin by recalling the definition of a scheme. A scheme is a pair $\left(X, \mathcal{O}_{X}\right)$, where $X$ is a topological space and $\mathcal{O}_{X}$ is a sheaf of rings on $X$, which is locally (on $X$ ) isomorphic to ( $\operatorname{Spec} A, \mathcal{O}_{\operatorname{Spec} A}$ ) for some commutative ring $A$. We seek a modification of this definition in which we allow $E_{\infty}$-rings to fill the role of ordinary commutative rings. The main challenge is to decide what we mean by $\operatorname{Spec} A$, when $A$ is an $E_{\infty}$-ring. We will adopt the following rather simple-minded definition:

Definition 2.2. Let $A$ be an $E_{\infty}$-ring. Then the topological space $\operatorname{Spec} A$, the Zariski-spectrum of $A$, is defined to be the spectrum of the ordinary commutative ring $\pi_{0} A$ : in other words, the set of prime ideals in $\pi_{0} A$. We endow $\operatorname{Spec} A$ with the usual Zariski-topology, with a basis of open sets given by the loci $U_{f}=$ $\{\mathfrak{p} \mid f \notin \mathfrak{p}\}, f \in \pi_{0} A$.

To complete the definition of the Zariski-spectrum of an $E_{\infty}$-ring $A$, we need to define the structure sheaf $\mathcal{O}_{\text {Spec } A}$. By general nonsense, it suffices to define $\mathcal{O}_{\operatorname{Spec} A}$ on each of the basic open subsets $U_{f} \subseteq \operatorname{Spec} A$. If $A$ were an ordinary commutative ring, we would define $\mathcal{O}_{\text {Spec } A}\left(U_{f}\right)=A\left[f^{-1}\right]$. This definition makes sense also in the $E_{\infty}$-context. Namely, given an $E_{\infty}$-ring $A$ and an element $f \in \pi_{0} A$, there exists a map of $E_{\infty}$-rings $A \rightarrow A\left[f^{-1}\right]$, which is characterized by either of the following equivalent assertions:

(1) The map $\pi_{*} A \rightarrow \pi_{*}\left(A\left[f^{-1}\right]\right)$ identifies $\pi_{*}\left(A\left[f^{-1}\right]\right)$ with $\left(\pi_{*} A\right)\left[f^{-1}\right]$.

(2) For any $E_{\infty}$-ring $B$, the induced map

$$
\operatorname{Hom}\left(A\left[f^{-1}\right], B\right) \rightarrow \operatorname{Hom}(A, B)
$$

is a homotopy equivalence of the left hand side onto the subspace of the right hand side consisting of all maps $A \rightarrow B$ which carry $f$ to an invertible element in $\pi_{0} B$ (this is a union of path components of $\operatorname{Hom}(A, B))$.

In virtue of the second characterization, the map $A \rightarrow A\left[f^{-1}\right]$ is well-defined up to canonical equivalence; moreover, it is sufficiently functorial to allow a definition of the structure sheaf $\mathcal{O}_{\operatorname{Spec} A}$.

We are now prepared to offer our main definition: 
Definition 2.3. A derived scheme is a topological space $X$ equipped with a sheaf of $E_{\infty}$-rings $\mathcal{O}_{X}$, which is locally equivalent to $\left(\operatorname{Spec} A, \mathcal{O}_{\operatorname{Spec} A}\right)$ where $A$ is an $E_{\infty}$-ring.

Remark 2.3. As we mentioned earlier, the world of $E_{\infty}$-rings is higher-categorical in nature. Consequently, one needs to be careful what one means by a sheaf of $E_{\infty}$-rings. There are several approaches to making this idea precise. One is to use Quillen's theory of model categories. Namely, one can let $\mathcal{C}$ be a suitable model-category for $E_{\infty}$-rings (for example, commutative monoids in symmetric spectra: see [17]). Now we can consider the category of $\mathcal{C}$-valued presheaves on a topological space $X$. This category of presheaves is itself endowed with a model structure, which simultaneously reflects the original model structure on $\mathcal{C}$ and the topology of $X$. Namely, we define a map $\mathcal{F} \rightarrow \mathcal{G}$ of presheaves to be a cofibration if it induces a cofibration $\mathcal{F}(U) \rightarrow \mathcal{G}(U)$ in $\mathcal{C}$ for every open subset $U \subseteq X$, and a weak equivalence if it induces a weak equivalence on stalks $\mathcal{F}_{x} \rightarrow \mathcal{G}_{x}$ for every point $x \in X$. One can then define a sheaf of $E_{\infty}$-rings on $X$ to be a fibrant and cofibrant object of this model category.

Remark 2.4. As with $E_{\infty}$-rings themselves, derived schemes are higher-categorical objects by nature. That means that given derived schemes $\left(X, \mathcal{O}_{X}\right)$ and $\left(Y, \mathcal{O}_{Y}\right)$, we can naturally associate a space of morphisms from $\left(X, \mathcal{O}_{X}\right)$ to $\left(Y, \mathcal{O}_{Y}\right)$. Namely, we define

$$
\operatorname{Hom}\left(\left(X, \mathcal{O}_{X}\right),\left(Y, \mathcal{O}_{Y}\right)\right)=\coprod_{f: X \rightarrow Y} \operatorname{Hom}_{0}\left(\mathcal{O}_{Y}, f_{*} \mathcal{O}_{X}\right) .
$$

Here $\operatorname{Hom}_{0}\left(\mathcal{O}_{Y}, f_{*} \mathcal{O}_{X}\right)$ is the subspace of $\operatorname{Hom}\left(\mathcal{O}_{Y}, f_{*} \mathcal{O}_{X}\right)$ consisting of local maps of sheaves of $E_{\infty}$-rings: that is, maps which induce local homomorphisms $\pi_{0} \mathcal{O}_{Y, f(x)} \rightarrow \pi_{0} \mathcal{O}_{X, x}$ of commutative rings for each $x \in X$. This is a union of path components of $\operatorname{Hom}\left(\mathcal{O}_{Y}, f_{*} \mathcal{O}_{X}\right)$, which may be defined following the description in Remark 2.3.

Example 2.1. Let $A$ be an $E_{\infty}$ ring. Then $\left(\operatorname{Spec} A, \mathcal{O}_{\operatorname{Spec} A}\right)$ is a derived scheme. Derived schemes which arise via this construction we will call affine.

Remark 2.5. Let $\left(X, \mathcal{O}_{X}\right)$ be an ordinary scheme. Since every ordinary commutative ring can be regarded as an $E_{\infty}$-ring, we may regard $\mathcal{O}_{X}$ as a presheaf of $E_{\infty}$-rings on $X$. This presheaf is generally not a sheaf: this is because of the existence of nontrivial cohomology groups of the structure sheaf $\mathcal{O}_{X}$ over the open subsets of $X$. Let $\mathcal{O}_{X}^{\prime}$ denote the sheafification of $\mathcal{O}_{X}$, in the setting of sheaves of $E_{\infty}$-rings. Then $\left(X, \mathcal{O}_{X}^{\prime}\right)$ is a derived scheme. We will abuse terminology by ignoring the distinction between $\mathcal{O}_{X}$ and $\mathcal{O}_{X}^{\prime}$ (either one can be recovered from the other, via the functors of sheafification and $\pi_{0}$. We also note that the map $\mathcal{O}_{X}(U) \rightarrow \mathcal{O}_{X}^{\prime}(U)$ is an equivalence whenever $U \subseteq X$ is affine). Thus, every ordinary scheme can be regarded as a derived scheme.

Conversely, let $\left(X, \mathcal{O}_{X}\right)$ be a derived scheme. Then the functor

$$
U \mapsto \pi_{0}\left(\mathcal{O}_{X}(U)\right)
$$

is a presheaf of commutative rings on $X$. We let $\pi_{0} \mathcal{O}_{X}$ denote the sheafification of this presheaf. (A vanishing theorem of Grothendieck ensures that $\left(\pi_{0} \mathcal{O}_{X}\right)(U) \simeq \pi_{0}\left(\mathcal{O}_{X}(U)\right)$ whenever $U$ is affine.) The pair $\left(X, \pi_{0} \mathcal{O}_{X}\right)$ is a scheme. We call it the underlying ordinary scheme of $\left(X, \mathcal{O}_{X}\right)$, and will occasionally denote it by $\left(X, \mathcal{O}_{X}\right)$.

Remark 2.6. If we were to employ only $E_{\infty}$-ring spaces, rather than arbitrary $E_{\infty}$-rings, in our definition of derived schemes, then the functor

$$
\left(X, \mathcal{O}_{X}\right) \rightarrow\left(X, \pi_{0} \mathcal{O}_{X}\right)
$$

would be a right adjoint to the inclusion functor from schemes to derived schemes. In other words, we would be able to regard $\left(X, \pi_{0} \mathcal{O}_{X}\right)$ as the maximal ordinary subscheme of the derived scheme $\left(X, \mathcal{O}_{X}\right)$. There is an analogous construction in ordinary algebraic geometry: every scheme possesses a maximal reduced subscheme.

Without connectivity assumptions, no such interpretation is possible: there is no map which directly relates $\left(X, \mathcal{O}_{X}\right)$ and $\left(X, \pi_{0} \mathcal{O}_{X}\right)$. 
Much of the formalism of ordinary algebraic geometry can be carried over to derived algebraic geometry, without essential change. For example, if $\left(X, \mathcal{O}_{X}\right)$ is a derived scheme, then one can consider quasi-coherent sheaves on $X$ : these are functors $\mathcal{F}$ which assign to every open subset $U \subseteq X$ a $\mathcal{O}_{X}(U)$-module spectrum $\mathcal{F}(U)$, which satisfy $\mathcal{F}(V) \simeq \mathcal{F}(U) \otimes_{\mathcal{O}_{X}(U)} \mathcal{O}_{X}(V)$ whenever $V \subseteq U$ are affine, and which satisfy an appropriate descent condition.

There is also a good theory of flatness in derived algebraic geometry:

Definition 2.4. Let $p:\left(X, \mathcal{O}_{X}\right) \rightarrow\left(Y, \mathcal{O}_{Y}\right)$ be a map of derived schemes. We will say that $p$ is flat if, for every pair of open affine subsets $U \subseteq X, V \subseteq Y$ such that $p(U) \subseteq V$, the induced map of $E_{\infty}$-rings

$$
\mathcal{O}_{Y}(V) \rightarrow \mathcal{O}_{X}(U)
$$

is flat (in the sense of Definition 2.1).

Remark 2.7. As in ordinary algebraic geometry, one can give various equivalent formulations of Definition 2.4: for example, testing flatness only on particular affine covers of $X$ and $Y$, or on stalks of the structure sheaves.

We note that if $p:\left(X, \mathcal{O}_{X}\right) \rightarrow\left(Y, \mathcal{O}_{Y}\right)$ is a flat map of derived schemes, then the underlying map of ordinary schemes $\left(X, \pi_{0} \mathcal{O}_{X}\right) \rightarrow\left(Y, \pi_{0} \mathcal{O}_{Y}\right)$ is flat in the sense of ordinary algebraic geometry. Conversely, if $\left(Y, \mathcal{O}_{Y}\right)$ is an ordinary scheme, then $p$ is flat if and only if $\left(X, \mathcal{O}_{X}\right)$ is an ordinary scheme and $p$ is flat when viewed as a map of ordinary schemes. In other words, the fibers of a flat morphism are classical schemes.

In many of the applications that we consider, we will need to deal with algebro-geometric objects of a more general nature than schemes. For example, the moduli stack $\mathcal{M}_{1,1}$ of elliptic curves cannot be represented by a scheme. However, $\mathcal{M}_{1,1}$ is an algebraic stack, in the sense of Deligne-Mumford. Algebraic stacks are usually defined to be a certain class of functors from commutative rings to groupoids; however, for Deligne-Mumford stacks one can also take a more geometric approach to the definition:

Definition 2.5. A Deligne-Mumford stack is a topos $X$ with a sheaf $\mathcal{O}_{X}$ of commutative rings, such that the pair $\left(X, \mathcal{O}_{X}\right)$ is locally (on $X$ ) isomorphic with ( $\operatorname{Spec} A, \mathcal{O}_{\operatorname{Spec} A}$ ), where $A$ is a commutative ring. Here Spec $A$ denotes the étale topos of $A$, and $\mathcal{O}_{\mathrm{Spec} A}$ its canonical sheaf of rings.

As with schemes, it is possible to offer a derived version of Definition 2.5, and thereby obtain a notion of derived Deligne-Mumford stack. All of the above discussion carries over to this more general context, without essential change. The reason for introducing this definition is that we have already encountered a very interesting example:

Example 2.2. Let $\mathcal{M}_{1,1}=\left(\mathcal{M}, \mathcal{O}_{\mathcal{M}_{1,1}}\right)$ denote the (ordinary) moduli stack of elliptic curves; here we let the symbol $\mathcal{M}$ denote the étale topos of the moduli stack. Let $\mathcal{O}_{\mathcal{M}}$ Der denote the sheaf of $E_{\infty}$-rings on $\mathcal{M}$ constructed in Theorem 1.1. (To be more precise, Theorem 1.1 constructs a presheaf of $E_{\infty}$-rings on a particular site for the topos $\mathcal{M}$, which extends in a canonical way to the sheaf $\mathcal{O}$.) Then the pair $\mathcal{M}^{\text {Der }}=(\mathcal{M}, \mathcal{O})$ is a derived Deligne-Mumford stack. We have $\pi_{0} \mathcal{O}_{\mathcal{M}^{\text {Der }}} \simeq \mathcal{O}_{\mathcal{M}_{1,1}}$, so that the underlying ordinary DeligneMumford stack of $\mathcal{M}^{\text {Der }}$ is the classical moduli stack $\mathcal{M}_{1,1}$ of elliptic curves.

We can view Example 2.2 as offering a geometric interpretation of Theorem 1.1: namely, Theorem 1.1 asserts the existence of a certain "derived" version of the moduli stack of elliptic curves. This turns out to be a very useful perspective, because the derived stack $\mathcal{M}^{\text {Der }}$ itself admits a moduli-theoretic interpretation. This observation leads both to a new construction of the sheaf $\mathcal{O}_{\mathcal{M}^{D e r}}$, and to a theory of equivariant elliptic cohomology. It also permits the study of elliptic cohomology using tools from derived algebraic geometry, which has many other applications. 


\section{Derived Group Schemes and Orientations}

In $\S 2$, we argued that for a (multiplicative) cohomology theory $A$, there is a relationship between equivariant versions of $A$ and group schemes over the commutative ring $A(*)$. To exploit this relationship, it is even better to have a group scheme $\mathbb{G}$ defined over $A$ itself. The language of derived algebraic geometry enables us to make sense of this idea. Namely, suppose that $A$ is an $E_{\infty}$-ring; then Definition 2.3 allows us to speak of derived $A$-schemes; that is, maps $\mathbb{G} \rightarrow \operatorname{Spec} A$ in the setting of derived schemes. But how can we make sense of a group structure on $\mathbb{G}$ ? The question is somewhat subtle, since derived schemes are most naturally viewed as higher-categorical objects.

Let us first consider the case of ordinary schemes. Let $p: \mathbb{G} \rightarrow X$ be a map of schemes. What does it mean to say that $\mathbb{G}$ is a (commutative) group scheme over $X$ ? One possibility is to require that $\mathbb{G}$ be a commutative group object in the category of schemes over $X$ : in other words, to require the existence of a multiplication map $\mathbb{G} \times_{X} \mathbb{G} \rightarrow \mathbb{G}$ satisfying various identities, which can be depicted as commutative diagrams. There is an alternative way of phrasing this, using Grothendieck's "functor of points" philosophy. Namely, given an $X$-scheme $q: S \rightarrow X$, we can define

$$
\mathbb{G}(S)=\{r \in \operatorname{Hom}(S, \mathbb{G}) \mid p \circ r=q\} .
$$

In other words, via the Yoneda embedding we may identify $\mathbb{G}$ with a functor from $X$-schemes to sets. To endow $\mathbb{G}$ with the structure of a commutative group object (over $X$ ) is to give a lifting of this functor to the category of abelian groups. Going still further, we can identify $\mathbb{G}$ with this lifting. From this point of view, a commutative group scheme over $X$ is a functor from $X$-schemes to abelian groups, such that the underlying functor from $X$-schemes to sets happens to be representable by a scheme.

We can apply the same philosophy to derived algebraic geometry. However, there is one important difference: if $X$ is a derived scheme, then derived schemes over $X$ should not be viewed as an ordinary category. Rather, they behave in a higher-categorical fashion, so that for a pair of derived $X$-schemes $S$ and $S^{\prime}$, the collection $\operatorname{Hom}_{X}\left(S, S^{\prime}\right)$ of commutative diagrams

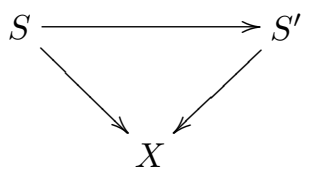

forms a space, rather than a set. However, this poses no major difficulties and we can make the following definition:

Definition 3.1. Let $X$ be a derived scheme. A commutative $X$-group is a (topological) functor $\mathbb{G}$ from derived $X$-schemes to topological abelian groups, such that the composite functor

$$
\{X \text {-schemes }\} \rightarrow\{\text { topological abelian groups }\} \rightarrow\{\text { topological spaces }\}
$$

is representable (up to weak homotopy equivalence) by a derived $X$-scheme that is flat over $X$. If $X=$ Spec $R$, we will also say that $\mathbb{G}$ is a commutative $R$-group.

We will often abuse terminology and not distinguish between a commutative $X$-group $\mathbb{G}$ over $X$ and the derived $X$-scheme that represents its underlying space-valued functor.

Remark 3.1. Let $X$ be an ordinary scheme. Then we may regard $X$ as a derived scheme, and a commutative $X$-group in the sense of Definition 3.1 is the same thing as a commutative group scheme that is flat over $X$, in the sense of ordinary algebraic geometry. More generally, any commutative $X$-group gives rise to a flat commutative group scheme over $\bar{X}$, the underlying ordinary scheme of $X$.

If we were to remove the flatness hypothesis from Definition 3.1, neither of the above statements would be true. The problem is that the formation of fiber products $\mathbb{G} \times_{X} \mathbb{G}$ is not compatible with passage between schemes and derived schemes, unless we assume that $\mathbb{G} \rightarrow X$ is flat. 
Let us now return to the study of equivariant cohomology. Suppose that we are given a cohomology theory which is represented by an $E_{\infty}$-ring $A$, and that we are looking for a definition of " $S$-equivariant $A$-cohomology." An $S^{1}$-equivariant cohomology theory determines an ordinary cohomology theory, simply by restricting attention to spaces on which $S^{1}$ acts trivially. In the best of all possible worlds, this cohomology theory might itself be representable by an $E_{\infty}$-ring $A_{S^{1}}$. In the case of complex $K$-theory, this $E_{\infty}$-ring can be described as the ring of functions on a commutative $K$-group $\mathbb{G}_{m}$ (we will analyze this example in detail in $§ 3.1$ ). In the general case, we will take the commutative $A$-group $\mathbb{G}$ as our starting point, and try to recover a theory of equivariant $A$-cohomology from it.

Of course, the commutative $A$-group $\mathbb{G}$ is not arbitrary, because any reasonable theory of equivariant $A$-cohomology can be compared with Borel-equivariant $A$-cohomology. In particular, there should be an "completion" map

$$
A_{S^{1}} \rightarrow A^{\mathrm{CP}^{\infty}} \text {. }
$$

In the example of $K$-theory, this map can be interpreted as a restriction map, from regular functions defined on all of $\mathbb{G}_{m}$ to formal functions defined only near the identity section of $\mathbb{G}_{m}$. Morally speaking, this is induced by a map

$$
\operatorname{Spf} A^{\mathrm{CP}^{\infty}} \rightarrow \mathbb{G}
$$

where the left hand side is a formal commutative $A$-group. In the case where $A$ is even and periodic, it is possible to make sense of the formal spectrum of $A^{\mathrm{CP}^{\infty}}$ using a formal version of derived algebraic geometry. Fortunately, this turns out to be unnecessary: it is possible to formulate the relevant structure in simpler terms. In order to do this, we first note that $\mathbf{C P}^{\infty}$ has the structure of a topological abelian group. For example, we can define a continuous multiplication on $\mathbf{C P}^{\infty}$ by realizing $\mathbf{C P}^{\infty}$ as the space of 1-dimensional complex subspaces of the polynomial ring $\mathbf{C}(x)$ (the multiplication on $\mathbf{C}(x)$ then determines a multiplication on $\mathbf{C P}^{\infty}=(\mathbf{C}(x)-\{0\}) / \mathbf{C}^{*}$.

Remark 3.2. Strictly speaking, the realization of $\mathbf{C P}^{\infty}$ as $(\mathbf{C}(x)-\{0\}) / \mathbf{C}^{*}$ does not endow $\mathbf{C} \mathrm{P}^{\infty}$ with the structure of a topological abelian group, because the inverse map is not continuous. However, it is homotopy equivalent to a topological abelian group (for example, it is an Eilenberg-MacLane space and can therefore be obtained as the geometric realization of a simplicial abelian group). We will henceforth ignore this minor difficulty, and simply refer to $\mathbf{C P}^{\infty}$ as a topological abelian group.

Definition 3.2. Let $X$ be a derived scheme, and let $\mathbb{G}$ be a commutative $X$-group. A preorientation of $\mathbb{G}$ is a map of topological abelian groups

$$
\mathrm{CP}^{\infty} \rightarrow \mathbb{G}(X) .
$$

A preoriented $X$-group is a commutative $X$-group $\mathbb{G}$ together with a preorientation of $\mathbb{G}$.

Suppose that $X$ is the spectrum of an $E_{\infty}$-ring $A$ and let $\mathbb{G}$ be a commutative $X$-group, with $A_{S^{1}}$ the $E_{\infty}$-ring of global functions on $\mathbb{G}$. Given an $X$-valued point of $\mathbb{G}$, restriction of functions induces a map $A_{S^{1}} \rightarrow A$. If $\mathbb{G}$ is equipped with a preorientation, then we get a collection of maps $A_{S^{1}} \rightarrow A$ indexed by $\mathbf{C P}^{\infty}$, which we may identify with a map $A_{S^{1}} \rightarrow A^{\mathbf{C P}^{\infty}}$; this is the "completion" map we are looking for. The requirement that $\mathbf{C P}^{\infty} \rightarrow \mathbb{G}(X)$ be a map of topological abelian groups corresponds to the condition that the map

$$
\operatorname{Spf} A^{\mathrm{CP}^{\infty}} \rightarrow \mathbb{G}
$$

should be compatible with the group structures.

It is possible to formulate the notion of a preorientation in even simpler terms. We regard $\mathbf{C P}^{\mathbf{D}}$ as the set of nonzero elements in the field of rational functions $\mathbf{C}(x)$, modulo scaling. Let $\mathbf{C P}^{n}$ denote the subspace corresponding to nonzero polynomials of degree $\leq n$. Then $\mathbf{C P}^{0}$ is the identity element of $\mathbf{C P}^{\infty}$. By the fundamental theorem of algebra, every nonzero element of $\mathbf{C}(x)$ factors as a product of (powers of) linear factors, which are unique up to scaling. Consequently, $\mathbf{C P}^{\infty}$ is generated, as an abelian group, by the subspace $\mathbf{C P}^{1}$. Moreover, it is almost freely generated: the only relation is that the point $\mathbf{C P}^{0} \subseteq \mathbf{C P}^{1}$ be the identity element. It follows that giving a preorientation $\mathbf{C P}^{\infty} \rightarrow \mathbb{G}(X)$ of a derived commutative group 
scheme over $X$ is equivalent to giving a map from the 2-sphere $\mathbf{C P}^{1}$ into $\mathbb{G}(X)$, which carries the base point to the identity element of $\mathbb{G}(X)$. Thus, up to homotopy, preorientations of $\mathbb{G}$ are classified by elements of the homotopy group $\pi_{2} \mathbb{G}(X)$.

Of course, given any commutative $A$-group $\mathbb{G}$, one can always find a preorientation of $\mathbb{G}$ : namely, the zero map. In order to rule out this degenerate example, we need to impose a further condition. Let us return to the example of complex $K$-theory, and let $\mathbb{G}_{m}=\operatorname{Spec} K_{S^{1}}$ the multiplicative group over $K$. In this case, $K^{\mathbf{C P}^{\infty}}$ is precisely the $E_{\infty}$-ring of formal functions on $\mathbb{G}_{m}$ near the identity section. In other words, our preorientation of $\mathbb{G}_{m}$ gives rise to a map

$$
s: \operatorname{Spf} K^{\mathbf{C P}^{\infty}} \rightarrow \mathbb{G}_{m}
$$

which is as nontrivial as possible: it realizes the left hand side as the formal completion $\widehat{\mathbb{G}}_{m}$ of $\mathbb{G}_{m}$. We wish to axiomatize this condition, without making reference to formal derived geometry. To do so, we note that in the example above, both $\operatorname{Spf} K^{\mathrm{CP}^{\infty}}$ and $\widehat{\mathbb{G}}_{m}$ are 1-dimensional formal groups. Consequently, to test whether or not $s$ is an isomorphism of formal groups, it suffices to check that the derivative of $s$ is invertible. In order to discover the object that plays the role of this derivative, we need to introduce a few more definitions.

Let $A$ be an $E_{\infty}$-ring and $\mathbb{G}$ a preoriented $A$-group. Let $\mathbb{G}_{0}$ denote the underlying ordinary scheme of $\mathbb{G}$. Let $\Omega_{\mathbb{G}_{0} / \pi_{0} A}$ denote the sheaf of differentials of $\mathbb{G}_{0}$ over $\pi_{0} A$, and let $\omega$ denote the pullback of this sheaf along the identity section

$$
\operatorname{Spec} \pi_{0} A \rightarrow \mathbb{G}_{0} \text {. }
$$

We will identify $\omega$ with the $\pi_{0} A$-module of global sections of $\omega$. In the case where $\mathbb{G}_{0}$ is smooth, we can identify $\omega$ with the dual of the (abelian) Lie algebra of $\mathbb{G}_{0}$.

Let $\sigma: S^{2} \rightarrow \mathbb{G}(A)$ be the preorientation of $\mathbb{G}$. Let $\operatorname{Spec} B=U \subseteq \mathbb{G}$ be an affine open subscheme of $\mathbb{G}$ containing the identity section; then we may identify $\sigma$ with a map $S^{2} \rightarrow U(A)$. Since $U$ is affine, we may identify this with a map of $E_{\infty}$-rings $B \rightarrow A^{S^{2}}$. This in turn induces a map of ordinary $\pi_{0} A$-algebras $\pi_{0} B \rightarrow A\left(S^{2}\right) \simeq \pi_{0} A \oplus \pi_{2} A$. The first component is a ring homomorphism $\pi_{0} B \rightarrow \pi_{0} A$ corresponding to the identity section of $\mathbb{G}_{0}$, while the second is a $\pi_{0} A$-algebra derivation of $\pi_{0} B$ into $\pi_{2} A$. This derivation is classified by a map $\beta: \omega \rightarrow \pi_{2} A$ of $\pi_{0} A$-modules.

Definition 3.3. Let $A$ be an $E_{\infty}$-ring and $\mathbb{G}$ a commutative A-group equipped with a preorientation $\sigma: S^{2} \rightarrow$ $\mathbb{G}(A)$. We will say that $\sigma$ is an orientation if the following conditions are satisfied:

(1) The underlying map of ordinary schemes $\mathbb{G}_{0} \rightarrow \operatorname{Spec} \pi_{0} A$ is smooth of relative dimension 1.

(2) The map $\beta: \omega \rightarrow \pi_{2} A$ induces isomorphisms

$$
\pi_{n} A \otimes_{\pi_{0} A} \omega \rightarrow \pi_{n+2} A
$$

for every integer $n \in \mathbf{Z}$.

An oriented A-group is a commutative A-group equipped with an orientation. More generally, if $X$ is a derived scheme, an oriented $X$-group is a preoriented $X$-group whose restriction to every open affine Spec $A \subseteq X$ is an oriented A-group.

Remark 3.3. Let $A$ be an $E_{\infty}$-ring and $\mathbb{G}$ an oriented $A$-group. Then condition (2) of Definition 3.3 forces $A$ to be weakly periodic. Conversely, if $A$ is weakly periodic, then condition (2) is equivalent to the assertion that $\beta$ is an isomorphism.

\subsection{Orientations of the Multiplicative Group}

Let $A$ be an $E_{\infty}$-ring. In the last section, we introduced the notion of a (pre)orientation on a commutative $A$-group. In this section, we specialize to the case where $\mathbb{G}$ is the multiplicative group $\mathbb{G}_{m}$.

As in ordinary algebraic geometry, we can make sense of the multiplicative group $\mathbb{G}_{m}$ over any $E_{\infty}$ ring $A$. In order to do so, we begin with a few general remarks about group algebras. Let $R$ be a commutative ring, 
and $M$ an abelian group. Then the group ring $R[M]$ can be characterized by the following universal property: to give a commutative ring homomorphism $R[M] \rightarrow S$, one must give a commutative ring homomorphism $R \rightarrow S$, together with a homomorphism from $M$ to the multiplicative group of $S$. We note that $R[M]$ is not merely an $R$-algebra, but an $R$-Hopf algebra. More precisely, Spec $R[M]$ is a commutative group scheme over $R$, the group structure coming from the fact that the collection of homomorphisms from $M$ to $S^{\times}$forms a group under pointwise multiplication, for every $R$-algebra $S$.

The above discussion generalizes without essential change to derived algebraic geometry. Given an $E_{\infty}$ $\operatorname{ring} A$ and a topological abelian group $M$, we can form a group algebra $A[M]$. In the special case where $M$ is the group $\mathbf{Z}$ of integers, we may informally write $A[\mathbf{Z}]$ as $A\left[t, t^{-1}\right]$ and we define the multiplicative group $\mathbb{G}_{m}$ to be Spec $A[\mathbf{Z}]$. We note that $\pi_{*}(A[\mathbf{Z}])=\left(\pi_{*} A\right)\left[t, t^{-1}\right]$. In particular, the multiplicative group $\mathbb{G}_{m}$ is flat over $\operatorname{Spec} A$, so we may regard it as a commutative $A$-group. The underlying ordinary scheme of $\mathbb{G}_{m}$ is just the usual multiplicative group over $\operatorname{Spec} \pi_{0} A$; in particular, it is smooth of relative dimension 1.

Let us consider the problem of constructing a preorientation of the multiplicative group $\mathbb{G}_{m}$. By definition, this is given by a homomorphism of topological abelian groups, from $\mathbf{C P}^{\infty}$ into $\mathbb{G}_{m}(A)$. This also can be rewritten in terms of group algebras: it is the same thing as a map of $A$-algebras from $A\left[\mathbf{C P}^{\infty}\right]$ into $A$.

Let $S$ denote the sphere spectrum: this is the initial object in the world of $E_{\infty}$-rings. To give an $A$-algebra map from $A[M]$ into $B$ is equivalent to giving a map of $E_{\infty}$-rings from $S[M]$ into $B$. In particular, to give a preorientation of the multiplicative group $\mathbb{G}_{m}$ over $A$ is equivalent to giving a map of $E_{\infty}$-rings from $S\left[\mathbf{C P}^{\infty}\right]$ into $A$. In other words, the $E_{\infty}$-ring $S\left[\mathbf{C P}^{\infty}\right]$ classifies preorientations of $\mathbb{G}_{m}$.

Remark 3.4. The group algebra $S\left[\mathbf{C P}^{\infty}\right]$ is more typically denoted by $\Sigma^{\infty} \mathbf{C P}_{+}^{\infty}$, and is called the (unreduced) suspension spectrum of the space $\mathbf{C P}^{\infty}$.

Let us now suppose that we have a preorientation $\sigma$ of $\mathbb{G}_{m}$ over an $E_{\infty}$-ring $A$, classified by a map $S\left[\mathbf{C P}^{\infty}\right] \rightarrow A$. The underlying ordinary scheme of $\mathbb{G}_{m}$ is the ordinary multiplicative group $\operatorname{Spec}\left(\pi_{0} A\right)\left[t, t^{-1}\right]$, and the sheaf of differentials on this scheme has a canonical generator $\frac{d t}{t}$. The restriction $\omega$ of this sheaf along the identity section is again canonically trivial, and the map

$$
\omega \rightarrow \pi_{2} A
$$

induced by the preorientation $\sigma$ can be identified with an element $\beta_{\sigma} \in \pi_{2} A$. Examining Definition 3.3, we see that $\sigma$ is an orientation if and only if $\beta_{\sigma}$ is invertible.

We note that $\beta_{\sigma}$ is functorial: if we are given an $E_{\infty}$-map $f: A \rightarrow B$, then we get an induced preorientation $f^{*} \sigma$ of the multiplicative group over $B$, and $\beta_{f^{*} \sigma}$ is the image of $\beta_{\sigma}$ under the induced map

$$
\pi_{2} A \rightarrow \pi_{2} B \text {. }
$$

In particular, $\beta_{\sigma}$ itself is the image of a universal element $\beta \in \pi_{2} S\left[\mathbf{C P}^{\infty}\right]$ under the map $S\left[\mathbf{C P}^{\infty}\right] \rightarrow A$.

The identification of $\beta \in \pi_{2} S\left[\mathbf{C P}^{\infty}\right]$ is a matter of simple calculation. The group algebra $S\left[\mathbf{C P}^{\infty}\right]$ can be identified with an $E_{\infty}$-ring space, and this space admits a canonical (multiplicative) map from $\mathbf{C P}^{\infty}$. The class $\beta$ can be identified with the composite map

$$
S^{2} \simeq \mathrm{CP}^{1} \subseteq \mathbf{C P}^{\infty} \rightarrow S\left[\mathbf{C P}^{\infty}\right] .
$$

(At least up to translation: this composite map carries the base point of $S^{2}$ to the multiplicative identity of $S\left[\mathbf{C P}^{\infty}\right]$, rather than the additive identity.)

To classify orientations on $\mathbb{G}_{m}$, we need to "invert" the element $\beta$ in $S\left[\mathbf{C P}{ }^{\infty}\right]$. In order words, we want to construct a map of $E_{\infty}$-rings $f: S\left[\mathbf{C P}^{\infty}\right] \rightarrow S\left[\mathrm{CP}^{\infty}\right]\left[\beta^{-1}\right]$ such that, for every $E_{\infty}$-ring $A$, composition with $f$ induces a homotopy equivalence of $\operatorname{Map}\left(S\left[\mathbf{C P}^{\infty}\right]\left[\beta^{-1}\right], A\right)$ with the subspace of $\operatorname{Map}\left(S\left[\mathbf{C P}^{\infty}\right], A\right)$ consisting of maps which carry $\beta$ to an invertible element in $\pi_{*} A$. This is a bit more subtle. It is easy to show that an $E_{\infty}$-ring with the desired universal property exists. With some additional work, one can show that it has the expected structure: that is, the natural map

$$
\left(\pi_{*} S\left[\mathbf{C P}^{\infty}\right]\right)\left[\beta^{-1}\right] \rightarrow \pi_{*}\left(S\left[\mathbf{C P}^{\infty}\right]\left[\beta^{-1}\right]\right)
$$

is an isomorphism. The structure of this $E_{\infty}$-ring is the subject of the following theorem of Snaith: 
Theorem 3.1. The $E_{\infty}$-ring $S\left[\mathbf{C P}^{\infty}\right]\left[\beta^{-1}\right]$ is equivalent to (periodic) complex $K$-theory.

Remark 3.5. The original formulation of Theorem 3.1 does not make use of the theory of $E_{\infty}$-rings. However, it is easy to construct an $E_{\infty}$ map $S\left[\mathbf{C P}^{\infty}\right]\left[\beta^{-1}\right] \rightarrow K$ (in our language, this map classifies the orientation of $\mathbb{G}_{m}$ over $K$-theory), and the real content of Snaith's theorem is that this map is an equivalence.

Remark 3.6. In Example 1.4, we saw that Landweber's theorem could be used to produce complex $K$-theory, as a cohomology theory, starting with purely algebraic data. We can view Theorem 3.1 as a much more sophisticated version of the same idea: we now recover complex $K$-theory as an $E_{\infty}$-ring, as the solution to a moduli problem. We will see later that the moduli-theoretic interpretation of Theorem 3.1 leads to purely algebraic constructions of equivariant complex $K$-theory as well.

Remark 3.7. The topological $\mathbf{C P}^{\infty}$ is a classifying space for complex line bundles; it is therefore natural to imagine that the points of $\mathbf{C P}^{\infty}$ are complex lines. Following this line of thought, we can imagine a similar description of the $E_{\infty}$-ring space $S\left[\mathbf{C P}^{\infty}\right]$ : points of $S\left[\mathbf{C P}^{\infty}\right]$ are given by formal sums of complex lines. Of course, this space is very different from the classifying space $\mathbf{Z} \times B U$ for complex $K$-theory, whose points are given by (virtual) vector spaces. The content of Theorem 3.1 is that this difference disappears when the Bott element is inverted. A very puzzling feature of Theorem 3.1 is the apparent absence of any direct connection of the theory of vector bundles with the problem of orienting the multiplicative group.

Remark 3.8. According to Theorem 3.1, the $E_{\infty}$-ring $K$ classifies orientations of the multiplicative group $\mathbb{G}_{m}$. However, one could consider a more general problem of orienting a commutative $A$-group $q: \mathbb{G} \rightarrow \operatorname{Spec} A$ which happened to look like the multiplicative group $\mathbb{G}_{m}$, in the sense that the underlying ordinary group scheme $\mathbb{G}_{0}$ is a 1 -dimensional torus over Spec $\pi_{0} A$. It turns out that this problem is not really more general: as in ordinary algebraic geomety, tori are rigid, so that $\mathbb{G}$ is isomorphic to the usual multiplicative group $\mathbb{G}_{m}$ after passing to a double cover of Spec $A$. In other words, we can understand all of the relevant structure by thinking about the usual multiplicative group $\mathbb{G}_{m}$ together with its automorphism group, which is cyclic of order 2. The automorphism group also acts on the classifying $E_{\infty}$-ring $K$, and this action corresponds to the operation of complex conjugation (on complex vector bundles).

In other words, by thinking not only about the multiplicative group but all multiplicative groups, we can recover not only complex $K$-theory but also real $K$-theory.

\subsection{Orientations of the Additive Group}

In $\S 3.1$ we studied the problem of orienting the multiplicative group $\mathbb{G}_{m}$. In this section, we wish to discuss the analogous problem for the additive group $\mathbb{G}_{a}$. Our first task is to define what we mean by the additive group $\mathbb{G}_{a}$.

One choice would be to define $\mathbb{G}_{a}$ so as to represent the functor which carries an $E_{\infty}$-ring $A$ to its underlying "additive group". However, we immediately encounter two problems. First, the addition on $A$ is generally not commutative enough: we can regard $A$ has having an "underlying space" which is an infinite loop space, but this underlying space is generally not homotopy equivalent to a topological abelian group. We can construct a derived group scheme which represents this functor: let us denote it by $\mathbb{A}^{1}$. But the group structure on $\mathbb{A}^{1}$ is not sufficiently commutative to carry out the constructions we will need in $\S 3.3$.

A second problem is that the derived scheme $\mathbb{A}^{1}$ is generally not flat over $A$. The $A$-scheme $\mathbb{A}^{1}$ may be written as Spec $A\{X\}$, where $A\{X\}$ denotes the free $E_{\infty}$-algebra generated over $A$ by one indeterminate $X$. However, the homotopy groups of $A\{X\}$ are perhaps not what one would naively expect: one has

$$
\pi_{k} A\{X\}=\bigoplus_{n \geq 0} A^{-k}\left(B \Sigma_{n}\right)
$$

Here $\Sigma_{n}$ denotes the symmetric group on $n$ letters. This calculation coincides with the naive expectation $\left(\pi_{k} A\right)[X]$ if and only if, for every $n \geq 0$, the classifying space $B \Sigma_{n}$ is acyclic with respect to $A$-cohomology. 
Remark 3.9. We would encounter the same difficulties if we used the "naive" procedure above to define the multiplicative group. Namely, there is a derived scheme $\mathrm{GL}_{1}$, which associates to every $E_{\infty}$-ring $A$ the underlying "multiplicative group" of $A$. This derived scheme $\mathrm{GL}_{1}$ is defined over the sphere spectrum $S$. However, it is not a commutative $S$-group in the sense of Definition 3.1, because it is neither flat over $S$ nor can it be made to take values in topological abelian groups. In particular, it does not coincide with the commutative $S$-group $\mathbb{G}_{m}$ defined in $\S 3.1$. Instead, there is a natural map

$$
\mathbb{G}_{m} \rightarrow \mathrm{GL}_{1}
$$

which is an equivalence over the rational numbers $\mathbf{Q}$. In general, one may regard $\mathbb{G}_{m}$ as universal among commutative $S$-groups admitting a homomorphism to $\mathrm{GL}_{1}$.

Since defining the additive group $\mathbb{G}_{a}$ over a general $E_{\infty}$-ring $A$ seems to be troublesome, we will choose a less ambitious starting point. We can certainly make sense of the ordinary additive group $\mathbb{G}_{a}=\operatorname{Spec} \mathbf{Z}[X]$ over the ring of integers $\mathbf{Z}$. This is a commutative group scheme over the ordinary scheme $\operatorname{Spec} \mathbf{Z}$. Since it is flat over $\mathbf{Z}$, we may also regard it as a commutative $\mathbf{Z}$-group in the sense of Definition 3.1. (We note that the ordinary commutative ring $\mathbf{Z}[X]$, when regarded as an $E_{\infty}$-algebra over $\mathbf{Z}$, is not freely generated by $X$ : this is the difference between $\mathbb{G}_{a}$ and the derived scheme $\mathbb{A}^{1}$ considered above).

Let us now suppose we are given a map of $E_{\infty}$-rings $\mathbf{Z} \rightarrow R$, and consider the problem of finding a preorientation of $\mathbb{G}_{a}$ over $R$ : in other words, the problem of finding a homomorphism of topological abelian groups $\mathbf{C P}^{\infty} \rightarrow \mathbb{G}_{a}(R)$. As in the case of the multiplicative group $\mathbb{G}_{m}$, this is equivalent to giving a map of $E_{\infty}$-rings $A \rightarrow R$, for a certain $\mathbf{Z}$-algebra $A$. A calculation similar to the one given in $\S 3.1$ allows us to identify $A$ with the group algebra $\mathbf{Z}\left[\mathbf{C P}^{\infty}\right]$; this is an $E_{\infty}$-ring with homotopy groups given by

$$
\pi_{*} \mathbf{Z}\left[\mathbf{C P}^{\infty}\right]=\mathrm{H}_{*}\left(\mathbf{C P}^{\infty} ; \mathbf{Z}\right)
$$

where multiplication is given by the Pontryagin product. As a ring, $\pi_{*} \mathbf{Z}\left[\mathbf{C P}^{\infty}\right]$ is a free divided power series algebra over $\mathbf{Z}$, on a single generator $\beta \in \pi_{2} \mathbf{Z}\left[\mathbf{C P}^{\infty}\right]$.

A preorientation $\sigma: \mathbf{Z}\left[\mathbf{C P}^{\infty}\right] \rightarrow R$ of the additive group over $R$ is an orientation if and only if $\sigma(\beta) \in \pi_{2} R$ is invertible. Consequently, the universal $E_{\infty}$-ring over which we have an orientation of $\mathbb{G}_{a}$ is the localization

$$
\mathbf{Z}\left[\mathbf{C P}^{\infty}\right]\left[\beta^{-1}\right] \simeq \mathbf{Q}\left[\mathbf{C P}^{\infty}\right]\left[\beta^{-1}\right] \simeq K \otimes \mathbf{Q} .
$$

This is the $E_{\infty}$-ring which represents periodic rational cohomology.

Remark 3.10. Let us say that $A$ is a rational $E_{\infty}$-ring if there is a map of $E_{\infty}$-rings from the field $\mathbf{Q}$ to $A$ (such a map is automatically unique, up to a contractible space of choices). Equivalently, $A$ is rational if the $\operatorname{ring} \pi_{0} A$ is a vector space over $\mathbf{Q}$.

If $A$ is rational, then the difficulties in defining the additive group over $A$ dissolve: classifying spaces $B G$ for finite groups are acyclic with respect to $A$-cohomology, so the free $E_{\infty}$-algebra $A\{X\}$ has the expected homotopy groups and is flat over $A$; moreover, the underlying "space" of any $A$-algebra is (naturally) homotopy equivalent to a topological abelian group. Consequently, we get an equivalence $\mathbb{A}^{1} \simeq \mathbb{G}_{a}$ over $A$, so $\mathbb{A}^{1}$ has the structure of a commutative $A$-group.

The point of the above discussion is that one can make sense of the additive group $\mathbb{G}_{a}$ in a bit more generality. For our purposes, this turns out to be irrelevant: although $\mathbb{G}_{a}$ can be defined over $\mathbf{Z}$, it can only be oriented over $\mathbf{Q}$.

Remark 3.11. Like the multiplicative group $\mathbb{G}_{m}$, the additive group $\mathbb{G}_{a}$ has nontrivial automorphisms. Provided that we work over $\mathbf{Q}$, these are parametrized by the multiplicative group $\mathbb{G}_{m}$. Consequently, $\mathbb{G}_{m}$ acts also on the $E_{\infty}$-ring $\mathbf{Q}\left[\beta, \beta^{-1}\right]$ which classifies orientations of $\mathbb{G}_{a}$. Passing to invariants with respect to this action, we recover ordinary (nonperiodic) cohomology with coefficients in $\mathbf{Q}$. 


\subsection{The Geometry of Preorientations}

Throughout this section, we fix an $E_{\infty}$-ring $A$ and a commutative $A$-group $\mathbb{G}$. Supposing that there exists a good equivariant version of $A$-cohomology, we would expect that for any compact Lie group $G$ and any $G$ space $X$, the equivariant cohomology $A_{G}(X)$ is a module over $A_{G}(*)$. If $G=S^{1}$, then $A_{G}(*)$ can be identified with the ring of functions on $\mathbb{G}$; it is therefore natural to expect $A_{G}(X)$ to be obtained as the global sections of a quasi-coherent sheaf on $\mathbb{G}$. More generally, for every compact Lie group $G$ one can construct a derived scheme $M_{G}$ and obtain $A_{G}(*)$ as the $E_{\infty}$-algebra of functions on $M_{G}$, and $A_{G}(X)$ as the global sections of a certain quasi-coherent sheaf on $M_{G}$. In this section, we address the first step: constructing the derived scheme $M_{T}$ in the case where $T$ is a compact abelian Lie group.

Let $X^{*}(T)$ denote the character group $\operatorname{Hom}\left(T, S^{1}\right)$; then $X^{*}(T)$ is a finitely generated abelian group (isomorphic to $\mathbf{Z}^{n}$ if $T$ is connected). We can recover $T$ from its character group via the isomorphism $T \simeq \operatorname{Hom}\left(X^{*}(T), S^{1}\right)$ (a special case of the Pontryagin duality theorem).

We let $M_{T}$ denote the derived $A$-scheme which classifies maps of abelian groups from $X^{*}(T)$ into $\mathbb{G}$. In other words, for every commutative $A$-algebra $B$, the space of $B$-valued points $M_{T}(B)$ is homotopy equivalent to a space of homomorphisms from $X^{*}(T)$ into $\mathbb{G}(B)$ (if $T$ is not connected, the abelian group $X^{*}(T)$ is not a free abelian group and one must be careful to use the derived mapping space between the topological abelian groups).

Example 3.1. If $T=S^{1}$, then $M_{T}=\mathbb{G}$. More generally, if $T$ is an $n$-dimensional torus, then $M_{T}$ is the $n$-fold fiber power of $\mathbb{G}$ over $\operatorname{Spec} A$. In particular, $M_{\{e\}}=\operatorname{Spec} A$.

Example 3.2. Let $T$ be a cyclic group $\mathbf{Z} / n \mathbf{Z}$ of order $n$. Then $M_{T}$ is equivalent to the kernel $\mathbb{G}[n]$ of the multiplication-by- $n$-map

$$
\mathbb{G} \stackrel{n}{\rightarrow} \mathbb{G}
$$

Remark 3.12. If $\mathbb{G}$ is affine, then each $M_{T}$ is also affine: in this case, we can dispense with derived schemes entirely and work at the level of $E_{\infty}$-rings. However, we will be primary interested in the case of elliptic cohomology, where $\mathbb{G}$ is not affine and the geometric language is indispensable.

The geometric object $M_{T}$ is meant to encode the $T$-equivariant $A$-cohomology of a point. Let $* / T$ denote the orbifold quotient of a point by the group $T$. Then the $T$-equivariant $A$-cohomology of a point ought to be identified with the orbifold $A$-cohomology of $* / T$ : that is, it ought to depend only on $* / T$, and not on $T$ itself. In other words, it ought to be independent of the chosen basepoint of $* / T$.

We may rephrase the situation as follows. Given a commutative $A$-group $\mathbb{G}$, we have constructed a functor

$$
T \mapsto M_{T}
$$

from compact abelian Lie groups to derived $A$-schemes. We wish to factor this through the classifying space

functor. In other words, we want a functor $\widetilde{M}$, defined on the (topological) category of spaces of the form $B T$ ( $T$ a compact abelian Lie group), such that

$$
\widetilde{M}(B T) \simeq M_{T} .
$$

The above formula determines the behavior of $\widetilde{M}$ on objects. To finish the job, we note that the space of maps from $B T$ to $B T^{\prime}$ is homotopy equivalent to a product $B T^{\prime} \times \operatorname{Hom}\left(T, T^{\prime}\right)$ : the first factor is given by the image of the basepoint of $B T$ in $B T^{\prime}$, and the second factor is a model for the space of base-point-preserving maps from $B T$ to $B T^{\prime}$. The functor $M$ is already defined on the second factor. To complete the definition of $\widetilde{M}$, we need to produce a map

$$
B T^{\prime} \rightarrow \operatorname{Hom}\left(M_{T}, M_{T^{\prime}}\right)
$$

Moreover, this should be suitably functorial in $T$ and $T^{\prime}$. Functoriality in $T$ implies that we need only define this map in the universal case $T=\{e\}$; that is, we need to produce a map 


$$
\operatorname{Hom}\left(X^{*}\left(T^{\prime}\right), \mathbf{C P}^{\infty}\right) \simeq B T^{\prime} \rightarrow M_{T^{\prime}}(A)=\operatorname{Hom}\left(X^{*}\left(T^{\prime}\right), \mathbb{G}(A)\right) .
$$

This map is required to be functorial in the character group $X^{*}\left(T^{\prime}\right)$; it is therefore determined by its behavior in the universal case where $X^{*}\left(T^{\prime}\right)=\mathbf{Z}$. Consequently, we have sketched the proof of the following:

Proposition 3.1. Let $A$ be an $E_{\infty}$-ring, let $\mathbb{G}$ a commutative $A$-group and let $M_{T}$ be defined as above. Let $\mathcal{C}$ be the (topological) category of spaces having the homotopy type of BT, where $T$ is a compact abelian Lie group.

The following data are equivalent:

(1) Covariant (topological) functors $\widetilde{M}$ from $\mathcal{C}$ to derived A-schemes, together with functorial identifications $\widetilde{M}(B T) \simeq M_{T}$.

(2) Preorientations $\sigma: \mathbf{C P}^{\infty} \rightarrow \mathbb{G}(A)$ of $\mathbb{G}$.

Remark 3.13. It is possible to sharpen Proposition 3.1 further. The functor $T \mapsto M_{T}$ is one way of encoding the commutative group structure on $\mathbb{G}$. Consequently, specifying a preoriented $A$-group is equivalent to specifying a functor $\widetilde{M}$ from $\mathcal{C}$ to $A$-schemes, such that $\widetilde{M}$ preserves certain Cartesian diagrams. This point of view is relevant when it comes to studying elliptic cohomology over the compactified moduli stack of elliptic curves, where there is a similar functor $\widetilde{M}$ which does not commute with products.

\subsection{Equivariant $A$-Cohomology for Abelian Groups}

Let $A$ be an $E_{\infty}$-ring and $\mathbb{G}$ a preoriented $A$-group. In the last section, we constructed a derived $A$-scheme $M_{T}$, for every compact abelian Lie group $T$. Moreover, we showed that $M_{T}$ really depends only on the classifying space $B T$, and not on a choice of basepoint on $B T$.

Let $T$ be a compact abelian Lie group, and $X$ a space on which $T$ acts. We wish to define the $T$-equivariant cohomology group $A_{T}(X)$. In the case where $X$ is a point, we have already described the appropriate definition: we should take the global sections of the structure sheaf of the space $M_{T}$. More generally, we will obtain $A_{T}(X)$ as the global sections of a certain sheaf $\mathcal{F}_{T}(X)$ on $M_{T}$.

We will say that a $T$-space $X$ is finite if it admits a filtration

$$
\emptyset=X_{0} \subseteq X_{1} \subseteq \ldots \subseteq X_{n}=X
$$

where $X_{i+1}=X_{i} \coprod_{T / T_{0} \times S^{k}}\left(T / T_{0} \times D^{k+1}\right)$ is obtained from $X_{i}$ by attaching a $T$-equivariant cell $\left(T / T_{0} \times\right.$ $\left.D^{k+1}\right)$.

Theorem 3.2. There exists a collection of functors $\left\{\mathcal{F}_{T}\right\}$, defined for every compact abelian Lie group $T$ and essentially uniquely prescribed by the following properties:

(1) For every compact abelian Lie group $T$, the functor $\mathcal{F}_{T}$ is a contravariant functor from finite $T$-spaces to quasi-coherent sheaves on $M_{T}$, which carries $T$-equivariant homotopy equivalences to equivalences of quasi-coherent sheaves.

(2) For fixed $T$, the functor $\mathcal{F}_{T}$ carries finite homotopy colimits of $T$-spaces to homotopy limits of quasicoherent sheaves.

(3) If $X$ is a point, then $\mathcal{F}_{T}=\mathcal{O}_{M_{T}}$.

(4) Let $T \subseteq T^{\prime}$, let $X$ be a finite $T$-space, and $X^{\prime}=\left(X \times T^{\prime}\right) / T$ the induced finite $T^{\prime}$-space. Let $f: M_{T^{\prime}} \rightarrow M_{T}$ be the induced morphism of derived schemes. Then $\mathcal{F}_{T^{\prime}}\left(X^{\prime}\right)=f_{*} \mathcal{F}_{T}(X)$.

Here is a sketch of the proof: suppose we wish to define $\mathcal{F}_{T}(X)$, where $X$ is a finite $T$-space. Using (2), we can reduce to the case where $X$ is an individual cell: in fact, to the case where $X$ is a $T$-orbit $T / T_{0}$, where $T_{0} \subseteq T$ is a closed subgroup. By condition (4), we have $\mathcal{F}_{T}\left(T / T_{0}\right)=f_{*} \mathcal{F}_{T_{0}}(*)$, where $f: M_{T_{0}} \rightarrow M_{T}$ is the induced map. Finally, $\mathcal{F}_{T_{0}}(*)$ is determined by condition (3). 
Remark 3.14. We could extend the functor $\mathcal{F}_{T}$ formally to all $T$-spaces, but we have refrained from doing so because inverse limit constructions behave poorly in the setting of quasi-coherent sheaves on $M_{T}$. For general $T$-spaces, it is $T$-equivariant homology which has better formal properties at the level of sheaves on $M_{T}$.

Remark 3.15. Suppose that $X$ is a $T$-space on which $T$ acts transitively. Then $X$ is abstractly isomorphic to $T / T_{0}$, but the isomorphism is not canonical unless we specify a base point on $X$. Consequently, the identification $\mathcal{F}_{T}(X) \simeq f_{*} \mathcal{O}_{M_{T_{0}}}$ is not quite canonical either; however, the ambiguity that results from the failure to specify a base point on $X$ is precisely accounted for by the fact that $M_{T}$ is depends only on the classifying space $B T$. In other words, a preorientation of $\mathbb{G}$ is precisely the datum needed to make the above prescription work.

Remark 3.16. To flesh out our sketch of the proof of Theorem 3.2, we would need to sharpen requirements (1) through (4) somewhat. For example, the isomorphisms

$$
f_{*} \mathcal{F}_{T}(X) \simeq \mathcal{F}_{T^{\prime}}\left(X^{\prime}\right)
$$

should be suitably compatible with the formations of chains of subgroups $T \subseteq T^{\prime} \subseteq T^{\prime \prime}$. We will not spell out the precise axiomatics of the situation here.

We are now prepared to define equivariant $A$-cohomology. Namely, for any compact abelian Lie group $T$ and any finite $T$-space $X$, let $A_{T}(X)=\Gamma\left(M_{T}, \mathcal{F}_{T}(X)\right)$ be the global sections of the sheaf $\mathcal{F}_{T}(X)$. Then $A_{T}(X)$ is a cohomology theory defined on finite $T$-spaces. We may, if we wish, go further to extract cohomology groups via the formula

$$
A_{T}^{n}(X)=\pi_{-n} A_{T}(X) .
$$

The reader who is familiar with equivariant homotopy theory might, at this point, raise an objection. We have defined a cohomology theory on the category of $T$-spaces, which assigns to each finite $T$-space $X$ the abelian group $A_{T}^{0}(X)$. By general nonsense, this functor is represented by a $G$-space $Z(0)$. Moreover, the functors $A_{T}^{n}(X)$ are represented by spaces $Z(n)$, which are deloopings of $Z(0)$. In other words, $Z(0)$ is a $T$ equivariant infinite loop space. However, in equivariant stable homotopy theory one demands more: namely, one wishes to be able to deloop not only with respect to ordinary spheres, but also spheres with a nontrivial (linear) action of $T$. To extract the necessary deloopings, we need to introduce a few more definitions.

By construction, the sheaves $\mathcal{F}_{T}(X)$ are not merely sheaves of modules on $M_{T}$, but actually sheaves of $E_{\infty}$-algebras. By functoriality, there are natural maps

$$
\mathcal{F}_{T}(X) \rightarrow \mathcal{F}_{T}(X \times Y) \leftarrow \mathcal{F}_{T}(Y)
$$

and therefore a map $\mathcal{F}_{T}(X) \otimes \mathcal{F}_{T}(Y) \rightarrow \mathcal{F}_{T}(X \times Y)$.

Let $T$ be a compact abelian Lie group, and let $X_{0} \subseteq X$ be finite $T$-spaces. We define $\mathcal{F}_{T}\left(X, X_{0}\right)$ to be the fiber of the map

$$
\mathcal{F}_{T}(X) \rightarrow \mathcal{F}_{T}\left(X_{0}\right)
$$

The multiplicatation maps defined above extend to give maps $\mathcal{F}_{T}\left(X, X_{0}\right) \otimes \mathcal{F}_{T}(Y) \rightarrow \mathcal{F}_{T}\left(X \times Y, X_{0} \times Y\right)$.

Proposition 3.2. Suppose that $A$ is an $E_{\infty}$ ring and let $\mathbb{G}$ be an oriented A-group. Let $T$ be a compact abelian Lie group, let $V$ be a finite dimensional unitary representation of $T$, and let $S V \subseteq B V$ be the unit sphere and the unit ball in $V$, respectively. Then:

(1) The quasi-coherent sheaf $\mathcal{L}_{V}=\mathcal{F}_{T}(B V, S V)$ is a line bundle on $M_{T}$.

(2) For every finite $T$-space $X$, the natural map

$$
\mathcal{L}_{V} \otimes \mathcal{F}_{T}(X) \rightarrow \mathcal{F}_{T}(X \times B V, X \times S V)
$$

is an equivalence. 
To give the flavor of the proof of Proposition 3.2, let us consider the case where $T$ is the circle group, and $V$ its defining 1-dimensional representation. The sheaf $\mathcal{L}_{V}$ is defined to be the fiber of the map

$$
\mathcal{F}_{T}(B V) \rightarrow \mathcal{F}_{T}(S V)
$$

The $T$-space $B V$ is equivariantly contractible, and the $T$-space $S V$ is $T$-equivariantly homotopy equivalent to $T$ itself. Consequently, the sheaf on the left hand side is the structure sheaf $\mathcal{O}_{\mathbb{G}}$, and the sheaf on the right hand side is the structure sheaf of the identity section of $\mathbb{G}$. Thus, $\mathcal{F}_{T}(B V, S V)$ can be viewed as the ideal sheaf for the identity section of $\mathbb{G}$. Assertion (1) of Proposition 3.2 asserts that $\mathcal{L}_{V}$ is invertible: this follows from the assumption that $\mathbb{G}$ is oriented, which implies that the underlying ordinary scheme $\mathbb{G}_{0}$ is smooth of relative dimension 1 over $\operatorname{Spec} \pi_{0} A$.

Now let us suppose that $\mathbb{G}$ is an oriented derived commutative group scheme over an $E_{\infty}$-ring $A$. For every finite dimensional complex representation $V$ of a compact abelian Lie group $T$, we let $\mathcal{L}_{V}=\mathcal{F}_{T}(B V, S V)$ be the line bundle on $M_{T}$ whose existence is asserted by Proposition 3.2. There are natural maps

$$
\mathcal{L}_{V} \otimes \mathcal{L}_{V^{\prime}} \rightarrow \mathcal{L}_{V \oplus V^{\prime}}
$$

which are equivalences in view of assertion (2) of Proposition 3.2. Consequently, the definition $\mathcal{L}_{V}$ extends to the case where $V$ is a virtual representation of $T$.

For every finite $T$-space $X$ and every virtual representation $V$ of $T$, we define

$$
A_{T}^{V}(X)=\pi_{0} \Gamma\left(M_{T}, \mathcal{F}_{T}(X) \otimes \mathcal{L}_{V}^{-1}\right) .
$$

Each functor $X \mapsto A_{T}^{V}(X)$ is represented by a $T$-space $Z(V)$. If $V$ is an actual representation of $T$, then $Z(V)$ is, up to $T$-equivariant homotopy equivalence, a delooping of $Z(0)$ with respect to the 1-point compactification of $V$. Consequently, when $\mathbb{G}$ is oriented, then the above construction yields an actual $T$-equivariant cohomology theory, defined in degrees indexed by the virtual representations of $T$.

\subsection{The Nonabelian Case}

Let $A$ be an $E_{\infty}$-ring, and $\mathbb{G}$ an oriented $A$-group. In $\S 3.4$, we constructed an equivariant cohomology theory $A_{G}$ for every compact abelian Lie group $G$. We now wish to treat the case where $G$ is nonabelian. We will do so by formally extrapolating from the abelian case. Namely, we claim the following:

Proposition 3.3. There exists a family of functors $X \mapsto A_{G}(X)$, defined for all compact Lie groups $G$, and essentially characterized by the following properties:

(1) For every compact Lie group $G, A_{G}$ is a contravariant functor from $G$-spaces to spectra, which preserves equivalences.

(2) For every inclusion $H \subseteq G$ of compact Lie groups, there are natural equivalences

$$
A_{H}(X) \simeq A_{G}((X \times G) / H) .
$$

(3) The functor $A_{G}$ carries homotopy colimits of $G$-spaces to homotopy limits of spectra.

(4) In the case where $G$ is abelian and $X$ is a finite $G$-space, the functor $A_{G}$ coincides with the functor defined in $\S 3.4$.

(5) Let $E^{a b} G$ be a $G$-space with the following property: for any closed subgroup $H \subseteq G$, the set $\left(E^{a b} G\right)^{H}$ of $H$-fixed points of $Y$ is empty if $H$ is nonabelian, and weakly contractible if $H$ is abelian. Then, for any $G$-space $X$, the natural map

$$
A_{G}(X) \rightarrow A_{G}\left(X \times E^{a b} G\right)
$$

is an equivalence. 
We sketch the proof. By (5), we can reduce to defining $A_{G}(X)$ in the case where the action of $G$ on $X$ has only abelian stabilizer groups. Replacing $X$ by a $G$-cell complex if necessary, we can assume that $X$ is composed of cells modelled on $G / H$, where $H \subseteq G$ is abelian. Using property (3), we can reduce to the case where $X=G / H$. Property (2) then forces $A_{G}(X)=A_{H}(*)$, which is determined by property (4).

We remark that conditions (1) through (3) are obvious and natural demands to place on any good theory of equivariant cohomology. Condition (4) is an equally natural demand, given that we want to build on the definition that we have already given in the abelian case. Condition (5), on the other hand, is more mysterious. We could define a different version of equivariant $A$-cohomology if we were to replace (4) and (5) by the following conditions:

$\left(4^{\prime}\right)$ In the case where $G$ is trivial, the functor $A_{G}(X)$ coincides with the function spectrum $A^{X}$. $\left(5^{\prime}\right)$ For any $G$-space $X$, the natural map

$$
A_{G}(X) \rightarrow A_{G}(X \times E G)
$$

is an equivalence.

Properties (1) through $\left(5^{\prime}\right)$ characterize Borel-equivariant cohomology. Condition (5) is considerably weaker than condition $\left(5^{\prime}\right)$, but it is still a rather severe assumption. It asserts that the equivariant cohomology theory associated to a nonabelian group $G$ is formally determined by equivariant cohomology theories associated to abelian subgroups of $G$. In other words, $A_{G}(X)$ should be given by a Borel construction relative to abelian subgroups of $G$. Why should we expect a good equivariant version of $A$-cohomology to satisfy (5), when the analogous condition $\left(5^{\prime}\right)$ is unreasonable? We offer several arguments:

- In the case where $A$ is complex $K$-theory, and we take $\mathbb{G}$ to be the multiplicative group $\mathbb{G}_{m}$ with its natural orientation, the equivariant cohomology theories described by Proposition 3.3 coincide with ordinary equivariant $K$-theory, even for nonabelian groups. In other words, assumption (5) above is satisfied in the case of complex $K$-theory.

- In the case where $G$ is abelian and $X$ is a finite $G$-space, the constructions of $\S 3.4$ give much more than the $G$-equivariant cohomology theory $A_{G}$. Namely, we had also a geometric object $M_{G}$, and an interpretation of $A_{G}(X)$ as the global sections of a certain sheaf $\mathcal{F}_{G}$ on $M_{G}$. There is similar geometry associated to the case where $G$ is nonabelian, at least provided that $G$ is connected.

- Suppose that one can construct a derived scheme $M_{G}$ and a functor $\mathcal{F}_{G}$ as in $\S 3.3$ and $\S 3.4$, where $G$ is a nonabelian compact Lie group. Suppose further that Proposition 3.2 remains valid in this case. Then it is possible to prove that assumption (5) holds, using the method of complex-oriented descent (as explained, for example, in [16]).

- When $\mathbb{G}$ is an oriented elliptic curve and $G$ is a connected compact Lie group, the theory of $G$-equivariant $A$-cohomology described by Proposition 3.3 is closely related to interesting geometry, such as the theory of regular $G_{\mathbf{C}}$-bundles on elliptic curves and nonabelian theta functions.

\section{Oriented Elliptic Curves}

In $\S 3$, we described the theory of oriented $A$-groups $\mathbb{G}$, where $A$ is an $E_{\infty}$-ring. In this section, we consider the most interesting case: where $\mathbb{G}$ is an elliptic curve.

Definition 4.1. Let $A$ be an $E_{\infty}$-ring. An elliptic curve over $A$ is a commutative A-group $E \rightarrow \operatorname{Spec} A$, having the property that the underlying map $\bar{E} \rightarrow \operatorname{Spec} \pi_{0} A$ is an elliptic curve (in the sense of classical algebraic geometry).

Remark 4.1. If $A$ is an ordinary commutative ring, regarded as an $E_{\infty}$-ring, then Definition 4.1 is equivalent to the usual definition of an elliptic curve over $A$. 
Remark 4.2. In ordinary algebraic geometry, one need not take the group structure on an elliptic curve $E \rightarrow \operatorname{Spec} A$ as part of the data. The group structure on $E$ is uniquely determined, as soon as one specifies a base point $\operatorname{Spec} A \rightarrow E$. In derived algebraic geometry, this is generally not true: the group structure on $E$ is not determined by the underlying derived scheme, even after a base point has been specified.

We now come to the main result of this survey:

Theorem 4.1. Let $\mathcal{M}^{\text {Der }}=\left(\mathcal{M}, \mathcal{O}^{\text {Der }}\right)$ denote the derived Deligne-Mumford stack of Example 2.2. For every $E_{\infty}$-ring $A$, let $E(A)$ denote the classifying space for the (topological) category of oriented elliptic curves over $A$. Then there is a natural homotopy equivalence

$$
\operatorname{Hom}\left(\operatorname{Spec} A, \mathcal{M}^{\text {Der }}\right) \simeq E(A) .
$$

In other words, $\mathcal{M}^{\text {Der }}$ may be viewed as a moduli stack for oriented elliptic curves in derived algebraic geometry, just as its underlying ordinary stack classifies elliptic curves in classical algebraic geometry.

Remark 4.3. We have phrased Theorem 4.1 in reference to the work of Goerss-Hopkins-Miller, which establishes the existence and uniqueness of the structure sheaf $\mathcal{O}^{\text {Der }}$. However, our proof of Theorem 4.1 does not require that we know existence of $\mathcal{O}^{\text {Der }}$ in advance. Instead, we could begin by considering the functor

$$
A \mapsto E(A)
$$

and prove that it is representable by a derived Deligne-Mumford stack $\left(X, \mathcal{O}_{X}\right)$. The hard part is to show that $X$ is equivalent to the étale topos of the ordinary moduli stack of elliptic curves, and that $\mathcal{O}_{X}$ is a sheaf of $E_{\infty}$-rings which satisfies the conclusions of Theorem 1.1. Consequently, our method yields a new proof of the existence of $\mathcal{O}^{\text {Der }}$.

The rest of this section is devoted to sketching the proof of Theorem 4.1.

\subsection{Construction of the Moduli Stack}

Let $A$ be an $E_{\infty}$-ring. As in Theorem 4.1, we let $E(A)$ denote the classifying space of the (topological) category of oriented elliptic curves over $\operatorname{Spec} A$. The first step is to prove that the functor

$$
A \mapsto E(A)
$$

is representable by a derived Deligne-Mumford stack. As a first approximation, we let $E^{\prime}(A)$ denote the classifying space of the (topological) category of preoriented elliptic curves over $\operatorname{Spec} A$. Let $\mathcal{M}_{1,1}=\left(\mathcal{M}, \mathcal{O}_{\mathcal{M}_{1,1}}\right)$ denote the classical moduli stack of elliptic curves. We observe that every elliptic curve over an ordinary commutative ring $R$ admits a unique preorientation (namely, zero). Consequently, the restriction of $E^{\prime}$ to ordinary commutative rings is represented by $\mathcal{M}_{1,1}$. We now apply the following general representability result:

Proposition 4.1. Let $\mathcal{F}$ be a functor from connective $E_{\infty}$-rings to spaces. Suppose that $\mathcal{F}$ satisfies the following conditions:

(1) There exists a Deligne-Mumford stack $(X, \mathcal{O})$ which represents the restriction of $\mathcal{F}$ to ordinary commutative rings. In other words, for any commutative ring $R$, the space $\mathcal{F}(R)$ is homotopy equivalent to the classifying space of the groupoid $\operatorname{Hom}(\operatorname{Spec} R,(\mathcal{X}, \mathcal{O}))$.

(2) The functor $\mathcal{F}$ satisfies étale descent.

(3) The functor $\mathcal{F}$ has a well-behaved deformation theory.

Then there exists a derived Deligne-Mumford stack $(X, \widetilde{\mathcal{O}})$ which represents the functor $\mathcal{F}$. Moreover, $\widetilde{\mathcal{O}}(U)$ is a connective $E_{\infty}$-ring whenever $U$ is affine. 
Proof (Proof sketch:). In virtue of condition (2), the assertion is local on $X$. We may therefore reduce to the case where $(X, \mathcal{O})$ is isomorphic to Spec $R$, where $R$ is a commutative ring. The idea is to obtain $(X, \widetilde{\mathcal{O}})=\operatorname{Spec} \widetilde{R}$, where $\widetilde{R}$ is a connective $E_{\infty}$-ring with $\pi_{0} \widetilde{R}=R$. One builds $\widetilde{R}$ as the inverse limit of a convergent tower of approximations, which are constructed using condition (3). For details, and a discussion of the meaning of (3), we refer the reader to [20].

We wish to apply Proposition 4.1 to the functor $A \mapsto E^{\prime}(A)$. Condition (1) is clear: on ordinary commutative rings, $E^{\prime}$ is represented by the classical moduli stack $\mathcal{M}_{1,1}$. The remaining conditions are also not difficult to verify, using general tools provided by derived algebraic geometry. We conclude that there exists a derived Deligne-Mumford stack $\left(\mathcal{M}, \mathcal{O}^{\prime}\right)$ which represents the functor $A \mapsto E^{\prime}(A)$, at least when the $E_{\infty^{-}}$ ring $A$ is connective. However, using the fact that elliptic curves are flat over $A$, one can show that $E^{\prime}(A)$ is equivalent to $E^{\prime}\left(\tau_{>0} A\right)$, where $\tau_{>0} A$ is the connective cover of $A$. It follows that $\left(\mathcal{N}, \mathcal{O}^{\prime}\right)$ represents the functor $E^{\prime}$ for all $E_{\infty}$-rings $A$. Moreover, $\mathcal{M}$ is the étale topos of the ordinary moduli stack $\mathcal{M}_{1,1}$ of elliptic curves, $\pi_{0} \mathcal{O}^{\prime} \simeq \mathcal{O}_{\mathcal{M}_{1,1}}$, and $\pi_{i} \mathcal{O}^{\prime}$ is a quasi-coherent sheaf on $\mathcal{M}_{1,1}$ for $i>0$. With a bit more effort, one can show that each $\pi_{i} \mathcal{O}^{\prime}$ is a coherent sheaf on $\mathcal{M}_{1,1}$.

Let $\omega$ denote the line bundle on $\mathcal{M}_{1,1}$ which associates to each elliptic curve $E \rightarrow$ Spec $R$ the $R$-module of invariant differentials on $E$. The preorientation of the universal elliptic curve over $\left(\mathcal{M}, \mathcal{O}^{\prime}\right)$ gives rise to a map $\beta: \omega \rightarrow \pi_{2} \mathcal{O}^{\prime}$ of coherent sheaves on $\mathcal{M}$. We can now define a new sheaf of $E_{\infty}$-rings $\mathcal{O}$ on $\mathcal{M}$ by "inverting" $\beta$. This sheaf has the property that

$$
\pi_{n} \mathcal{O} \simeq \underset{k}{\lim }\left\{\pi_{n+2 k} \mathcal{O}^{\prime} \otimes \mathcal{O}_{\mathcal{M}_{1,1}} \omega^{-k}\right\}
$$

in the category of quasi-coherent sheaves on $\mathcal{M}_{1,1}$.

Remark 4.4. Let $U \rightarrow \mathcal{M}$ be affine, and suppose that the restriction of $\omega$ to $U$ is free. Then $\mathcal{O}^{\prime}(U)=A$ is a connective $E_{\infty}$-ring, and we may identify $\beta$ with an element of $\pi_{2} A$. By definition, $\mathcal{O}(U)$ is the $A$-algebra $A\left[\beta^{-1}\right]$ obtained by inverting $\beta$. We note that $\mathcal{O}$ is not a connective sheaf of $E_{\infty}$-rings; rather it is (locally) 2-periodic, by construction.

To complete the proof of Theorem 4.1, it will suffice to prove the following:

(1) For $n=2 k$, the natural map $\omega^{k} \rightarrow \pi_{n} \mathcal{O}$ is an isomorphism of quasi-coherent sheaves on $\mathcal{M}_{1,1}$.

(2) For $n=2 k+1$, the quasi-coherent sheaf $\pi_{n} \mathcal{O}$ is trivial.

Indeed, suppose that (1) and (2) are satisfied. We first observe that $(\mathcal{M}, \mathcal{O})$ is a derived Deligne-Mumford stack. The assertion is local on $\mathcal{M}$, so we may restrict ourselves to an étale $U \rightarrow \mathcal{M}$ such that $\left(U, \mathcal{O}_{\mathcal{M}}{ }_{1,1} \mid U\right)$ is affine and $\omega \mid U$ is trivial. In this case, $\left(U, \mathcal{O}_{\mathcal{M}_{1,1}} \mid U\right) \simeq \operatorname{Spec} R$, where $R$ is a commutative ring; $A=\mathcal{O}^{\prime}(U)$ is a connective $E_{\infty}$-ring with $\pi_{0} A \simeq R$, and we may identify the map $\beta$ with an element in $\pi_{2} A$. Then $\mathcal{O}(U)=A\left[\beta^{-1}\right]$. Condition (1) asserts that $R \simeq \pi_{0} A\left[\beta^{-1}\right]$, so that $(U, \mathcal{O} \mid U)$ is equivalent to $\operatorname{Spec} A\left[\beta^{-1}\right]$.

By construction, the derived Deligne-Mumford stack $(\mathcal{M}, \mathcal{O})$ represents the functor $A \mapsto E(A)$. To complete the proof, it suffices to show that $\mathcal{O}$ coincides with the sheaf $\mathcal{O}^{\text {Der }}$ of $E_{\infty}$-rings constructed by Goerss, Hopkins and Miller. For this, it suffices to show that over each affine $U=\operatorname{Spec} R$ of $\mathcal{M}$, the $E_{\infty}$-ring $\mathcal{O}(U)$ gives rise to the elliptic cohomology theory associated to the universal (classical) elliptic curve $\mathcal{E}_{U}$ over $U$. In other words, we need to produce an isomorphism of the formal spectrum of $\mathcal{O}(U)\left(\mathbf{C P}^{\infty}\right)$ with the formal completion of the underlying ordinary elliptic curve of $\mathcal{E}_{U}$. By construction, we have such an isomorphism not only at the level of classical formal groups, but at the level of derived formal groups.

It remains to prove that (1) and (2) are satisfied. To simplify the discussion, we will consider only condition (2): the first condition can be handled by a similar but slightly more complicated argument.

Let $n$ be an odd integer. We wish to show that colimit $\pi_{n} \mathcal{O}$ of the directed system

$$
\left\{\pi_{n+2 k} \mathcal{O}^{\prime} \otimes_{\mathcal{O}_{\mathfrak{M}_{1,1}}} \omega^{-k}\right\}
$$

is zero. Since $\pi_{n} \mathcal{O}$ is generated by the images of $\pi_{n+2 k} \mathcal{O}^{\prime} \otimes_{\mathcal{O}_{\mathfrak{M}_{1,1}}} \omega^{-k}$, it suffices to show that each of these images is zero. Replacing $n$ by $n+2 k$ if necessary, it suffices to show that $f: \pi_{n} \mathcal{O}^{\prime} \rightarrow \pi_{n} \mathcal{O}$ is the zero map. 
Let $\mathcal{F}$ be the image of $f$. Then $\mathcal{F}$ is a quotient of the coherent sheaf $\pi_{n} \mathcal{O}^{\prime}$, and therefore coherent. If $\mathcal{F} \neq 0$, then $\mathcal{F}$ has support at some closed point $\kappa: \operatorname{Spec} \mathbf{F}_{q} \rightarrow \mathcal{M}_{1,1}$. Consequently, to prove that condition (2) holds, it suffices to prove that (2) holds in a formal neighborhood of the point $\kappa$. In other words, we need not consider the entire moduli stack $\mathcal{M}_{1,1}$ of elliptic curves: it is sufficient to consider (a formal neighborhood of) a single elliptic curve defined over a finite field $\mathbf{F}_{q}$.

Remark 4.5. The above argument reduces the proof of Theorem 4.1 in characteristic zero to a statement in characteristic $p$. This reduction is not necessary: in characteristic zero, it is fairly easy to verify (1) and (2) directly. We sketch how this is done. Let $A$ be an $E_{\infty}$-algebra over the field $\mathbf{Q}$ of rational numbers. The theory of elliptic curves over $A$ then reduces to the classical theory of elliptic curves, in the sense that they are classified by maps $\operatorname{Spec} A \rightarrow \mathcal{M}_{1,1}$.

It follows that, rationally, $\mathcal{O}^{\prime}$ is an algebra over $\pi_{0} \mathcal{O}^{\prime} \simeq \mathcal{O}_{\mathcal{M}_{1,1}}$. Moreover, it is easy to work out the structure of this algebra: namely, $\pi_{*} \mathcal{O}^{\prime}$ is the symmetric algebra on $\omega$ over $\mathcal{O}_{\mathcal{M}_{1,1}}$. Consequently, we observe that the direct system $\left\{\omega^{-k} \otimes_{\mathcal{O}_{\mathfrak{M} 1,1}} \pi_{n+2 k} \mathcal{O}^{\prime}\right\}$ is actually constant for $n+2 k>0$, isomorphic to $\omega^{m}$ if $n=2 m$ is even and 0 otherwise.

If we do not work rationally, the directed system $\left\{\omega^{-k} \otimes_{\mathcal{O}_{\mathfrak{M}_{1,1}}} \pi_{n+2 k} \mathcal{O}^{\prime}\right\}$ is not constant. The sheaves $\pi_{n} \mathcal{O}^{\prime}$ are complicated and we do not know how to compute them individually; only in the limit do we obtain a clean statement.

\subsection{The Proof of Theorem 4.1: The Local Case}

In $\S 4.1$, we reduced the proof of Theorem 4.1 to a local calculation, which makes reference only to a formal neighborhood of a closed point $\kappa: \operatorname{Spec} \mathbf{F}_{q} \rightarrow \mathcal{M}_{1,1}$ of the moduli stack of elliptic curves. In this section, we will explain how to perform this calculation, by adapting the theory of $p$-divisible groups to the setting of derived algebraic geometry. Let $p$ be a prime number, fixed throughout this section.

Definition 4.2. Let $A$ be an $E_{\infty}$-ring. Let $\mathbb{G}$ be a functor from commutative A-algebras to topological abelian groups. We will say that $\mathbb{G}$ is a p-divisible group of height $h$ over $A$ if the following conditions hold:

(1) The functor $B \mapsto \mathbb{G}(B)$ is a sheaf (in the $\infty$-categorical sense) with respect to the flat topology on $A$ algebras.

(2) For each $n$, the multiplication map $p^{n}: \mathbb{G} \rightarrow \mathbb{G}$ is surjective (in the flat topology) with kernel $\mathbb{G}\left[p^{n}\right]$.

(3) The colimit of the system $\left\{\mathbb{G}\left[p^{n}\right]\right\}$ is equivalent to $\mathbb{G}$ (as sheaves of topological abelian groups with respect to the flat topology).

(4) For each $n \geq 0$, the functor $\mathbb{G}\left[p^{n}\right]$ is a commutative A-group, that is finite and flat over $A$ of rank $p^{n h}$.

Remark 4.6. If $A$ is an ordinary commutative ring, then Definition 4.2 recovers the usual notion of a $p$ divisible group over $A$. Since a $p$-divisible group $\mathbb{G}$ is determined by the flat derived $A$-schemes $\mathbb{G}\left[p^{n}\right]$, the theory of $p$-divisible groups over an arbitrary $E_{\infty}$-ring $A$ is equivalent to the theory of $p$-divisible groups over the connective cover $\tau_{\geq 0} A$.

Example 4.1. Let $E$ be an elliptic curve over an $E_{\infty}$-ring $A$. Let $E\left[p^{n}\right]$ denote the fiber of the map $p^{n}: E \rightarrow E$, and let $\mathbb{G}$ be the direct limit of the system $\left\{E\left[p^{n}\right]\right\}_{n \geq 0}$. Then $\mathbb{G}$ is a $p$-divisible group over $A$ of height 2 , which we will denote by $E\left[p^{\infty}\right]$.

Our approach to the proof of Theorem 4.1 rests on a derived analogue of the Serre-Tate theorem, which asserts that the deformation theory of an elliptic curve is equivalent to the deformation theory of its $p$-divisible group, provided that we work $p$-adically:

Theorem 4.2. Let $A$ be an $E_{\infty}$-ring. Suppose that $\pi_{0} A$ is a complete, local, Noetherian ring whose residue field $k$ has characteristic $p$, and that each of the homotopy groups $\pi_{n} A$ is a finitely generated module over $\pi_{0} A$. Then there is a Cartesian diagram (of $\infty$-categories) 


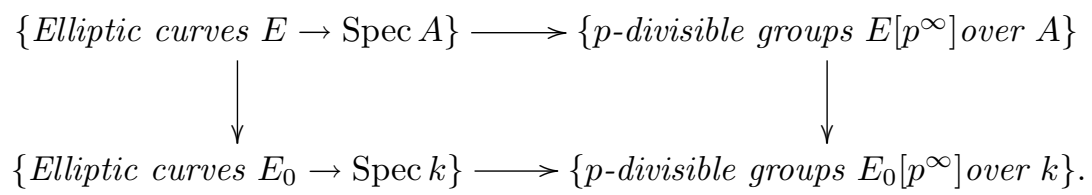

According to Theorem 4.2, giving an elliptic curve over $A$ is equivalent to giving an elliptic curve $E_{0}$ over the residue field $k$ of $\pi_{0} A$, together with a lifting of the $p$-divisible group $E_{0}\left[p^{\infty}\right]$ to a $p$-divisible group over $A$. In other words, the deformation theory of elliptic curves is the same as the deformation theory of their $p$-divisible groups (in characteristic $p$ ).

Let $A$ be an $E_{\infty}$-ring satisfying the hypotheses of Theorem 4.2. It is possible to analyze the theory of $p$-divisible groups over $A$ along the same lines as one analyzes the theory of $p$-divisible groups over $\pi_{0} A$. Namely, every $p$-divisible group $\mathbb{G}$ over $A$ has a unique filtration

$$
\mathbb{G}_{\text {inf }} \rightarrow \mathbb{G} \rightarrow \mathbb{G}_{e t} .
$$

Here $\mathbb{G}_{\text {inf }}$ is a "purely infinitesimal" $p$-divisible group obtained from the $p$-power torsion points of a (uniquely determined) commutative formal $A$-group, and $\mathbb{G}_{e t}$ is an étale $p$-divisible group associated to a $p$-adic local system on Spec $A=\operatorname{Spec} \pi_{0} A$. As with commutative $A$-groups, we can speak of preorientations and orientations on a $p$-divisible group $\mathbb{G}$. A preorientation of $\mathbb{G}$ is equivalent to a preorientation of its infinitesimal part $\mathbb{G}_{\text {inf }}$. An orientation of $\mathbb{G}$ is an identification of $\mathbb{G}_{\text {inf }}$ with the $p$-power torsion on $\operatorname{Spf} A^{\mathrm{CP}^{\infty}}$; in particular, an orientation of $\mathbb{G}$ can exist only if $\mathbb{G}$ is 1 -dimensional.

Now suppose that $k$ is a perfect field of characteristic $p$, and that we are given a point $\kappa: \operatorname{Spec} k \rightarrow \mathcal{M}_{1,1}$ corresponding to an elliptic curve $E_{0}$ over $k$. Let $\mathcal{O}_{\kappa}^{\prime}$ denote the formal completion of the sheaf $\mathcal{O}^{\prime}$ at the point $\kappa$. Then $\mathcal{O}_{\kappa}^{\prime}$ is a connective $E_{\infty}$-ring such that $\pi_{0} \mathcal{O}_{\kappa}^{\prime}$ is the formal completion of the sheaf of functions on the classical moduli stack $\mathcal{M}_{1,1}$ at the point $\kappa$. There is a preoriented elliptic curve $E \rightarrow \operatorname{Spec} \mathcal{O}_{\kappa}^{\prime}$, which we may regard as the universal deformation of $E_{0}$ (as a preoriented elliptic curve). Let $\bar{E}$ denote the underlying ordinary scheme of $E$; the $\pi_{0} \mathcal{O}_{\kappa}^{\prime}$-module $\omega$ of invariant differentials on $\bar{E}$ is a free $\pi_{0} \mathcal{O}_{\kappa}^{\prime}$-module of rank 1 . Fixing a generator of $\omega$, the preorientation of $E$ gives an element $\beta \in \pi_{2} \mathcal{O}_{\kappa}^{\prime}$. Let $\mathcal{O}_{\kappa}=\mathcal{O}_{\kappa}^{\prime}\left[\beta^{-1}\right]$. As we saw in $\S 4.1$, Theorem 4.1 amounts to proving that $\mathcal{O}_{\kappa}$ is even and $\pi_{0} \mathcal{O}_{\kappa} \simeq \pi_{0} \mathcal{O}_{\kappa}^{\prime}$.

In view of Theorem 4.2, we may reinterpret the formal spectrum $\operatorname{Spf} \mathcal{O}_{\kappa}^{\prime}$ in the language of $p$-divisible groups. Namely, let $\mathbb{G}_{0}$ denote the $p$-divisible group of the elliptic curve $E_{0} \rightarrow$ Spec $k$. Since $k$ is an ordinary commutative ring, $\mathbb{G}_{0}$ has a unique preorientation. The formal spectrum $\operatorname{Spf} \mathcal{O}_{\kappa}^{\prime}$ is the parameter space for the universal deformation of $E_{0}$ as a preoriented elliptic curve. By Theorem 4.2, this is the also the parameter space for the universal deformation of $\mathbb{G}_{0}$ as a preoriented $p$-divisible group. More informally, we might also say that the $E_{\infty}$-ring $\mathcal{O}_{\kappa}$ classifies oriented p-divisible groups which are deformations of $\mathbb{G}_{0}$.

There are now two cases to consider, according to whether or not the elliptic curve $E_{0} \rightarrow$ Spec $k$ is supersingular. If $E_{0}$ is supersingular, then its $p$-divisible group $\mathbb{G}_{0}$ is entirely infinitesimal. It follows that any oriented deformation $E$ of $E_{0}$ over an $E_{\infty}$-ring $R$ is uniquely determined: $E$ is determined by its $p$-divisible group $E\left[p^{\infty}\right] \simeq\left(\operatorname{Spf} R^{\mathbf{C P}^{\infty}}\right)\left[p^{\infty}\right]$. In concrete terms, this translates into a certain mapping property of the deformation ring $\mathcal{O}_{\kappa}$. One can show that this mapping property characterizes the Lubin-Tate spectrum $E_{2}$ associated to the formal group $\widehat{E_{0}}$, which is known to have all of the desired properties. (We refer the reader to [26] for a proof of the Hopkins-Miller theorem on Lubin-Tate spectra, which is very close to establishing the universal property that we need here.)

If the elliptic curve $E_{0} \rightarrow$ Spec $k$ is not supersingular, then we need to work a bit harder. In this case, any $p$-divisible group $\mathbb{G}$ deforming $\mathbb{G}_{0}=E_{0}\left[p^{\infty}\right]$ admits a filtration

$$
\mathbb{G}_{\text {inf }} \rightarrow \mathbb{G} \rightarrow \mathbb{G}_{e t}
$$

where both $\mathbb{G}_{\text {inf }}$ and $\mathbb{G}_{e t}$ have height 1 . Consequently, to understand the deformation theory of $\mathbb{G}_{0}$ we must understand three things: the deformation theory of $\left(\mathbb{G}_{0}\right)_{\text {inf }}$, the deformation theory of $\left(\mathbb{G}_{0}\right)_{\text {et }}$, and the deformation theory of the space of extensions $\operatorname{Ext}\left(\mathbb{G}_{e t}, \mathbb{G}_{\text {inf }}\right)$. 
The deformation theory of the étale $p$-divisible group $\left(\mathbb{G}_{0}\right)_{e t}$ is easy. An étale $p$-divisible group over $A$ is given by a $p$-adic local system over $\operatorname{Spec} A$, which is the same thing as a $p$-adic local system on $\operatorname{Spec} \pi_{0} A$. If $A$ is a complete, local Noetherian ring with residue field $k$, these may be identified with $p$-adic local systems on Spec $k$ : in other words, finite free $\mathbf{Z}_{p}$-modules with a continuous action of the absolute Galois group of $k$. In particular, this description is independent of $A:\left(\mathbb{G}_{0}\right)_{e t}$ has a unique deformation to every formal thickening of $k$, even in derived algebraic geometry. To simplify the discussion which follows, we will suppose that $k$ is algebraically closed. Then we may identify $\left(\mathbb{G}_{0}\right)_{e t}$ with the constant (derived) group scheme $\mathbf{Q}_{p} / \mathbf{Z}_{p}$.

It is possible to analyze the infinitesimal part $\left(\mathbb{G}_{0}\right)_{\text {inf }}$ in the same way, using the fact that it is the dual of an étale $p$-divisible group and therefore also has a trivial deformation theory. However, this is not necessary: remember that we are really interested in deformations of $\mathbb{G}_{0}$ which are oriented. As we saw in the supersingular case, the orientation of $\mathbb{G}$ determines the infinitesimal part $\mathbb{G}_{\text {inf }}$, and the $E_{\infty}$-ring which controls the universal deformation of $\mathbb{G}_{\text {inf }}$ as an oriented $p$-divisible group is a Lubin-Tate spectrum $E_{1}$ (in this case, an unramified extension of the $p$-adic completion of complex $K$-theory).

Finally, we need to study the deformation theory $\operatorname{Ext}\left(\mathbf{Q}_{p} / \mathbf{Z}_{p}, \mathbb{G}_{\text {inf }}\right)$. As in classical algebraic geometry, one shows that in a suitable setting one has isomorphisms

$$
\mathbb{G}_{\text {inf }} \simeq \operatorname{Hom}\left(\mathbf{Z}_{p}, \mathbb{G}_{\text {inf }}\right) \simeq \operatorname{Ext}\left(\mathbf{Q}_{p} / \mathbf{Z}_{p}, \mathbb{G}_{\text {inf }}\right) .
$$

Consequently, to obtain the universal deformation $E_{\infty}$-ring of $\mathbb{G}_{0}$ as an oriented $p$-divisible group, one should take the $E_{\infty}$-ring of functions on the universal deformation of $\left(\mathbb{G}_{0}\right)_{\text {inf }}$ as an oriented $p$-divisible group. Since this deformation is oriented, we may identify this $E_{\infty}$-ring with $E_{1}^{\mathbf{C P}}{ }^{\infty}$. A simple computation now shows that $\mathcal{O}_{\kappa} \simeq E_{1}^{\mathbf{C P}^{\infty}}$ has the desired properties.

Remark 4.7. The arguments employed above are quite general, and can be applied to $p$-divisible groups which do not necessarily arise from elliptic curves. For example, one can produce "derived versions" of certain Shimura varieties, at least $p$-adically, using the same methods. For more details, we refer the reader to $[8]$.

\subsection{Elliptic Cohomology near $\infty$}

Classically, an elliptic curve $E \rightarrow$ Spec $\mathbf{C}$ is determined, up to noncanonical isomorphism, by its $j$-invariant $j(E) \in \mathbf{C}$. Consequently, the moduli space of elliptic curves is isomorphic to $\mathbf{C}$, which is not compact. One can compactify the moduli space by allowing elliptic curves to develop a nodal singularity.

We wish to extend the theory of elliptic cohomology to this compactification. To carry out this extension, it suffices to work locally in a formal neighborhood of $j=\infty$. Complex analytically, we can construct a family of elliptic curves over the punctured disk $\{q \in \mathbf{C}: 0<|q|<1\}$, which assigns to a complex parameter $q$ the elliptic curve $E_{q}=\mathbf{C}^{*} / q^{\mathbf{Z}}$. This family has a natural extension over the disk $\{q \in \mathbf{C}:|q|<1\}$, where $E_{q}$ specializes to a nodal rational curve at $q=0$. Provided that one is willing to work in a formal neighborhood of $q=0$, one can even give an algebraic construction of the elliptic curve $E_{q}$. This algebraic construction gives a generalized elliptic curve $T$, the Tate curve, which is defined over $\mathbf{Z}[[q]]$. The fiber of $T$ over $q=0$ is isomorphic to a nodal rational curve, and its general fiber "is" $\mathbb{G}_{m} / q^{\mathbf{Z}}$, in a suitable sense.

We will sketch a construction of the Tate curve which makes sense in derived algebraic geometry. For this, we will assume that the reader is familiar with the language of toric varieties (for a very readable account of the theory of toric varieties, we refer the reader to [15]).

Fix an $E_{\infty}$-ring $R$. Let $\Lambda$ be a lattice (that is, a free $\mathbf{Z}$-module of finite rank) and let $F=\left\{\sigma_{\alpha}\right\}_{\alpha \in A}$ be a rational polyhedral fan in $\mathbf{Z}$. For each $\alpha \in A$, let $\sigma_{\alpha}^{\vee} \subseteq \Lambda^{\vee}$ denote the dual cone to $\sigma_{\alpha}$, regarded as a commutative monoid. Then the monoid algebra $R\left[\sigma_{\alpha}^{\vee}\right]$ is an $E_{\infty}$-ring, and we may define $U_{\alpha}=\operatorname{Spec} R\left[\sigma_{\alpha}^{\vee}\right]$. The correspondence $\alpha \mapsto U_{\alpha}$ is functorial: it carries inclusions of cones to open immersions of affine derived schemes. We may therefore construct a derived scheme $X_{F}=\lim \left\{U_{\alpha}\right\}_{\alpha \in A}$ by gluing these affine charts together, using the pattern provided by the fan $F$. We call $X_{F}$ the toric variety over $R$ defined by $F$. When $R$ is an ordinary commutative ring, this is a well-known classical construction; it makes perfect sense in derived algebraic geometry as well. 
Let $F_{0}=\left\{\{0\}, \mathbf{Z}_{\geq 0}\right\}$ be the fan in $\mathbf{Z}$ giving rise to the toric variety $X_{F_{0}}=\operatorname{Spec} R\left[\mathbf{Z}_{\geq 0}\right]$. We will write the $E_{\infty}$-ring $R\left[\mathbf{Z}_{\geq 0}\right]$ as $R[q]$, though we should note that it is not the free $E_{\infty}$-ring on one generator over $R$ (the generator $q$ satisfies relations that force it to strictly commute with itself). For each $n \in \mathbf{Z}$, let $\sigma_{n}$ denote the cone

$$
\{(a, b) \in \mathbf{Z} \times \mathbf{Z} \mid n a \leq b \leq(n+1) a\},
$$

and let $F$ denote the fan in $\mathbf{Z} \times \mathbf{Z}$ consisting of the cones $\sigma_{n}$, together with all their faces. Projection onto the first factor gives a map $F \rightarrow F_{0}$ of fans, and therefore a map of toric varieties $f: X_{F} \rightarrow \operatorname{Spec} R[q]$. The fiber of $f$ over any point $q \neq 0$ can be identified with the multiplicative group $\mathbb{G}_{m}$, while the fiber of $f$ over the point $q=0$ is an infinite chain of rational curves, each intersecting the next in a node.

Consider the automorphism $\tau: \mathbf{Z} \times \mathbf{Z} \rightarrow \mathbf{Z} \times \mathbf{Z}$ defined by $\tau(a, b)=(a, b+a)$. It is clear that $\tau\left(\sigma_{n}\right)=\sigma_{n+1}$, so that $\tau$ preserves the fan $F$ and therefore defines an automorphism of $X_{F}$, which we shall also denote by $\tau$. The action of $\tau$ is not free: for $q_{0} \neq 0, \tau$ acts on the fiber $f^{-1}\left\{q_{0}\right\} \simeq \mathbb{G}_{m}$ by multiplication by $q_{0}$. However, the action of $\tau$ is free when it is restricted to the fiber $f^{-1}(0)$.

Let $\widehat{X}_{F}$ denote the formal completion of $X_{F}$ along the fiber $f^{-1}\{0\}$, and let $R[[q]]$ denote the formal completion of $R[q]$ along the closed subset defined by the equation $q=0$. The group $\tau^{\mathbf{Z}}$ acts freely on $\widehat{X}_{F}$, and thus we may define a formal derived scheme $\widehat{T}$ by taking the quotient of $\widehat{X}_{F}$ by the action of $\tau^{\mathbf{z}}$. We note that the fiber of $\widehat{T}$ over $q=0$ can be identified with a nodal rational curve; in particular, it is proper over Spec $R$. Using a generalization of the Grothendieck existence theorem to derived algebraic geometry, one can show that $\widehat{T}$ is the formal completion of a (uniquely determined) derived scheme $T \rightarrow \operatorname{Spec} R[[q]]$. We call $T$ the Tate curve; its restriction to the punctured formal disk $\operatorname{Spec} R((q))=\operatorname{Spec} R[[q]]\left[q^{-1}\right]$ is an elliptic curve over $R((q))$, which we may think of as the quotient of the multiplicative group $\mathbb{G}_{m}$ by the subgroup $q^{\mathbf{Z}}$.

Of course, we are primarily interested in oriented elliptic curves. The Tate curve is essentially given as a quotient of the multiplicative group $\mathbb{G}_{m}$. In particular, its formal completion is equivalent to the formal completion of the multiplicative group. Consequently, giving an orientation of the Tate curve is equivalent to giving an orientation of the multiplicative group $\mathbb{G}_{m}$. By Theorem 3.1, this is equivalent to working over the $E_{\infty}$-ring $K$ (the complex $K$-theory spectrum). In other words, the Tate curve $T$ over $K((q))$ is an oriented elliptic curve, which is therefore classified by a map

$$
\operatorname{Spec} K((q)) \rightarrow \mathcal{M}^{\text {Der }}
$$

Of course, there are additional symmetries which we should take into account. The involution $(a, b) \mapsto(a,-b)$ preserves the fan $F$ and intertwines with the action of $\tau$, and therefore induces an involution on $T$ (the inverse map with respect to the group structure). This involution preserves the orientation on $T$, provided that we allow it to act also on the ground ring $K$. We therefore actually obtain a map Spec $K((q)) /\{ \pm 1\} \rightarrow \mathcal{M}^{\text {Der }}$, where the group $\{ \pm 1\}$ operates on $K$ by complex conjugation.

One can define a new derived Deligne-Mumford stack by forming a pushout square

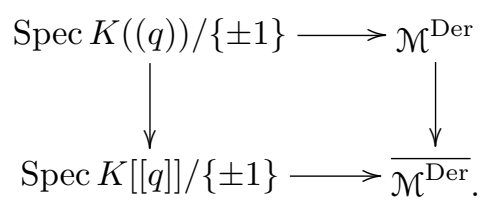

Here $\overline{\mathcal{M}^{\text {Der }}}$ is a compactification of the derived moduli stack $\mathcal{M}^{\text {Der }}$. The underlying ordinary Deligne-Mumford stack of $\overline{\mathcal{M}^{\text {Der }}}$ is the classical Deligne-Mumford compactification of $\mathcal{M}_{1,1}$.

Remark 4.8. Much of the theory of elliptic cohomology carries over to the compactified moduli stack $\overline{\mathcal{M}^{\text {Der }}}$. However, there are often subtleties at $\infty$. For example, we can glue the universal oriented elliptic curve $\mathcal{E}$ over $\mathcal{M}^{\text {Der }}$ with the Tate curve $T$, to obtain a universal oriented generalized elliptic curve $\bar{\varepsilon}$ over $\overline{\mathcal{M}^{\text {Der }}}$. However, $\bar{\varepsilon}$ is not a derived group scheme over $\overline{\mathcal{M}^{\text {Der }}}$ : it has a group structure only on the smooth locus of the map $\bar{\varepsilon} \rightarrow \overline{\mathcal{M}^{\text {Der }}}$. 
Consequently, the construction of geometric objects that we described in $\S 3.3$ needs to be modified. Since $\bar{\varepsilon}$ is not a derived group scheme, we cannot define the geometric object $M_{G}$ associated to a compact abelian Lie group $G$ to be the derived scheme $\operatorname{Hom}\left(G^{\vee}, \bar{\varepsilon}\right)$. It is possible to make the necessary modifications, giving an explicit construction of $M_{G}$ near $\infty$ using the theory of toric varieties. However, the construction is somewhat complicated and we will not describe it here.

\section{Applications}

\subsection{2-Equivariant Elliptic Cohomology}

Let $A$ be an $E_{\infty}$-ring and let $E \rightarrow$ Spec $A$ be an oriented elliptic curve. In $\S 3.3$ we used this data to construct a derived $A$-scheme $M_{G}$, for every compact abelian Lie group $G$, which is the natural "home" for the $G$ equivariant version of $A$-cohomology described in $\S 3.4$. It is possible to construct a derived scheme $M_{G}$ with similar properties even when $G$ is nonabelian. If $G$ is connected and $T$ is a maximal torus of $G$, the derived scheme $M_{G}$ looks roughly like a quotient of $M_{T}$ by the action of the Weyl group of $G$. In particular, when $G$ is connected, $M_{G}$ is a derived scheme whose underlying classical scheme can be identified with the moduli

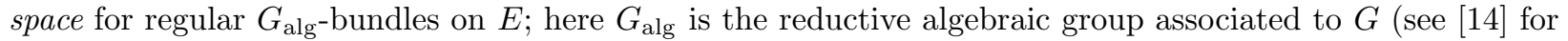

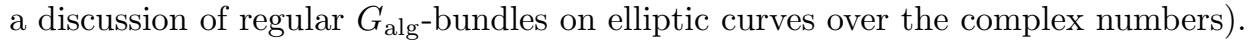

Remark 5.1. It is possible to describe $M_{S U(n)}$ and $M_{U(n)}$ more explicitly, in the language of derived algebraic geometry. However, we do not know of any moduli-theoretic interpretation of the derived scheme $M_{G}$ in general. This seems to be a difficult question, primarily because the underlying classical object is a moduli space rather than a moduli stack.

One way of thinking about equivariant elliptic cohomology is that it is a correspondence which associates derived schemes to certain topological spaces. In particular, to the classifying space $B G$ it assigns the derived scheme $M_{G}$. It is possible to extend this process to a more general class of topological spaces, to obtain what we call 2-equivariant elliptic cohomology. For an explanation of this terminology we refer the reader to $\S 5.4$. The correspondence associated to an oriented elliptic curve $E \rightarrow$ Spec $A$ is summarized in the following table.

\begin{tabular}{cc} 
Topological Space & Associated Geometric Object \\
\hline$*$ & $\operatorname{Spec} A$ \\
$\mathbf{C P}$ & $E$ \\
$B \mathbf{Z} / n \mathbf{Z}$ & $E[n]$ \\
$B G$ & $M_{G}$ \\
$K(\mathbf{Z} / n \mathbf{Z}, 2)$ & $\mu_{n}$ \\
$K(\mathbf{Z}, 3)$ & $\mathbb{G}_{m}$ \\
$K(\mathbf{Z}, 4)$ & $B \mathbb{G}_{m}$
\end{tabular}

Every level $l \in \mathrm{H}^{4}(B G, \mathbf{Z})$ classifies a fibration

$$
K(\mathbf{Z}, 3) \rightarrow X \rightarrow B G,
$$

and 2-equivariant elliptic cohomology associates to this a fibration of geometric objects: in other words, a principal $\mathbb{G}_{m}$ over $M_{G}$, which we may identify with a line bundle $\mathcal{L}_{l}$ over $M_{G}$. 
Remark 5.2. The line bundle $\mathcal{L}_{l}$ gives rise to a line bundle on the classical moduli space $\bar{M}_{G}$ of regular $G$-bundles on elliptic curves. This is the "theta" bundle whose global sections are nonabelian $\theta$-functions: in other words, the spaces of conformal blocks for the modular functor underlying the Wess-Zumino-Witten model.

Example 5.1. Let us say that a commutative $A$-group $X \rightarrow \operatorname{Spec} A$ is an abelian scheme over $\operatorname{Spec} A$ if the underlying map of ordinary schemes $\bar{X} \rightarrow$ Spec $\pi_{0} A$ is an abelian scheme, in the sense of classical algebraic geometry. There is a good duality theory for abelian schemes: namely, given an abelian scheme $X \rightarrow \operatorname{Spec} A$, one can define a dual abelian scheme $X^{\vee}$ which classifies extensions of $X$ by the multiplicative group $\mathbb{G}_{m}$.

In classical algebraic geometry, every elliptic curve is canonically isomorphic to its dual. The analogous result is not true in derived algebraic geometry. However, it is true for oriented elliptic curves $E \rightarrow \operatorname{Spec} A$. Let $l: \mathbf{C P}^{\infty} \times \mathbf{C P}^{\infty} \rightarrow K(\mathbf{Z}, 4)$ classify the cup-product operation

$$
\mathrm{H}^{2}(X ; \mathbf{Z}) \times \mathrm{H}^{2}(X ; \mathbf{Z}) \rightarrow \mathrm{H}^{4}(X ; \mathbf{Z}) .
$$

Then $\mathcal{L}_{l}$ is a line bundle on the product $E \times_{\operatorname{Spec} A} E$. The symmetry and bi-additivity of the cup product operation translate into the assertion that $\mathcal{L}_{l}$ is a symmetric biextension of $E$ by $\mathbb{G}_{m}$ (see [9] for a discussion in the classical setting). This gives an identification of $E$ with the dual elliptic curve $E^{\vee}$.

We will not describe the construction of 2-equivariant elliptic cohomology here. However, we should point out that it is essentially uniquely determined by the properties we have asserted above: namely, that it is a functorial process which assigns to each level $l: B G \rightarrow K(\mathbf{Z}, 4)$ a line bundle $\mathcal{L}_{l}$ on $M_{G}$, and that it determines an identification of the elliptic curve $E$ with its dual.

\subsection{Loop Group Representations}

Let us consider a punctured formal disk around $\infty$ on the moduli stack of elliptic curves. As we saw in $\S 4.3$, over this disk elliptic cohomology is given by the $E_{\infty}$-ring $K((q))$, where $K$ is complex $K$-theory. In other words, near $\infty$, elliptic cohomology reduces to $K$-theory. However, equivariant elliptic cohomology does not reduce to equivariant $K$-theory. Both of these equivariant theories are given by the constructions of $\S 3$. However, the inputs to these constructions are different: to get equivariant $K$-theory, we use the multiplicative group $\mathbb{G}_{m}$; for elliptic cohomology, we use the Tate curve $\mathbb{G}_{m} / q^{\mathbf{Z}}$. Thus, we should expect $G$ equivariant $K$-theory to be somewhat less complicated than $G$-equivariant elliptic cohomology. The former is related to the representation theory of the group $G$, but the latter is related to the representation theory of the loop group $L G$.

We begin with a quick review of the theory of loop groups: for a more extensive discussion, we refer the reader to [24]. Fix a connected compact Lie group $G$, which for simplicity we will assume to be simple. Then the group $\mathrm{H}^{4}(B G ; \mathbf{Z})$ is canonically isomorphic to $\mathbf{Z}$. Let us fix a nonnegative integer $l$, which we identify with an element of $\mathrm{H}^{4}(B G ; \mathbf{Z})$ and therefore with a map $B G \rightarrow K(\mathbf{Z}, 4)$. Let $L G$ denote the loop group of (smooth) loops $S^{1} \rightarrow G$. The level $l$ classifies a central extension

$$
S^{1} \rightarrow \widetilde{L G} \rightarrow L G .
$$

Moreover, there is an action of $S^{1}$ on the group $\widetilde{L G}$, which descends to the natural $S^{1}$-action on $L G$ given by rotation of loops. We let $\widetilde{L G}+$ denote the semidirect product of $\widetilde{L G}$ by $S^{1}$, via this action. We will refer to the circle of this semidirect product decomposition as the energy circle, and the circle $S^{1} \subseteq \widetilde{L G} \rightarrow \widetilde{L G}^{+}$ as the central circle.

Definition 5.1. Let $V$ be a (Hilbert) representation of $\widetilde{L G}^{+}$. We will say that $V$ is a positive energy representation of level $l$ if it satisfies the following conditions:

(1) The central circle $S^{1} \subseteq \widetilde{L G}^{+}$acts on $V$ via the defining character $S^{1} \hookrightarrow \mathbf{C}^{*}$. 
(2) The Hilbert space $V$ decomposes as a direct sum of finite-dimensional eigenspaces $V_{(n)}$ with respect to the action of the energy circle.

(3) The energy eigenspaces $V_{(n)}$ are zero for $n \ll 0$.

Remark 5.3. If we ignore the action of the energy circle, then there are finitely many irreducible positive energy representations of $\widetilde{L G}$, up to isomorphism. However, each of these extends to a representation of $\widetilde{L G}^{+}$in infinitely many ways: given any positive energy representation $V$ of $\widetilde{L G}^{+}$, we can obtain a new positive energy representation $V(1)$ by tensoring with the defining representation of the energy circle (in other words, by shifting the energy).

Remark 5.4. Every positive energy representation $V$ of $\widetilde{L G}^{+}$can be decomposed as a direct sum of irreducible representations $V_{\alpha}$. The irreducible constituents $V_{\alpha}$ may be infinite in number, so long as the lowest energy of $V_{\alpha}$ tends to $\infty$ with $\alpha$.

Consider the Tate curve $E$ over $K((q))$, constructed in $\S 4.3$. Using the constructions described in $\S 5.1$ we saw that there is a derived scheme $M_{G} \rightarrow$ Spec $K((q))$ and a line bundle $\mathcal{L}_{l}$ on $M_{G}$, naturally associated to the level $l: B G \rightarrow K(\mathbf{Z}, 4)$. The global sections $\Gamma\left(M_{G}, \mathcal{L}_{l}\right)$ can be identified with an $K((q))$-module, which we may informally refer to as the G-equivariant elliptic cohomology of a point, at level $l$.

We can now state the relationship between elliptic cohomology and the theory of loop group representations:

Theorem 5.1. Let $G$ be a compact simple Lie group and $l$ a nonnegative level on $G$. There is a natural identification of $\Gamma\left(M_{G}, \mathcal{L}_{l}\right)$ with the $K$-theory of the (topological) category of positive energy representations of $\widetilde{L G}^{+}$at level $l$. Under this correspondence, multiplication by $q \in \pi_{0} K((q))$ corresponds to the energy shift $V \mapsto V(1)$.

The classical analogue of this result, which identifies the $K$-group of positive energy representations of $\widetilde{L G}^{+}$with the global sections of the theta bundle over the underlying ordinary scheme of $M_{G}$, is proven in $[3]$.

Remark 5.5. Theorem 5.1 is related to the work of Freed, Hopkins and Telemann (see [13]), which identifies the $K$-theory of loop group representations with the twisted $G$-equivariant $K$-theory of the group $G$ itself.

\subsection{The String Orientation}

Let $V$ be a finite dimensional real vector space of dimension $d \geq 5$, equipped with a positive definite inner product. The orthogonal group $\mathrm{O}(V)$ of automorphisms of $V$ is not connected. Consequently, we define $\mathrm{SO}(V) \subseteq \mathrm{O}(V)$ to be the connected component of the identity; the map $\mathrm{SO}(V) \subseteq \mathrm{O}(V)$ may be regarded as "killing" $\pi_{0} \mathrm{O}(V)$, in the sense that $\mathrm{SO}(V)$ is a connected space with $\pi_{n} \mathrm{SO}(V) \simeq \pi_{n} \mathrm{O}(V)$ an isomorphism for $n>0$.

The group $S O(V)$ is not simply connected, but it has a simply connected double cover $\operatorname{Spin}(V)$. The map $\operatorname{Spin}(V) \rightarrow \mathrm{SO}(V)$ has the effect of "killing" $\pi_{1} \mathrm{SO}(V)$, in the sense that $\pi_{n} \operatorname{Spin}(V) \rightarrow \pi_{n} \mathrm{SO}(V)$ is an isomorphism for $n \neq 1$, but $\operatorname{Spin}(V)$ is simply connected.

An algebraic topologist would see no reason to stop there. The group $\pi_{2} \operatorname{Spin}(V) \simeq \pi_{2} \mathrm{SO}(V) \simeq \pi_{2} \mathrm{O}(V)$ is trivial, but the homotopy group $\pi_{3} \operatorname{Spin}(V) \simeq \pi_{3} \mathrm{SO}(V) \simeq \pi_{3} \mathrm{O}(V)$ is (canonically) isomorphic to $\mathbf{Z}$. We may therefore construct a map String $(V) \rightarrow \operatorname{Spin}(V)$ which induces an isomorphism $\pi_{n} \operatorname{String}(V) \rightarrow \pi_{n} \operatorname{Spin}(V)$ for $n \neq 3$, but such that $\pi_{3} \operatorname{String}(V)=0$.

Remark 5.6. One might wonder what sort of an object $\operatorname{String}(V) i s$. It is certainly not a finite dimensional Lie group. One can construct a topological space with the desired properties using standard methods of homotopy theory (attaching cells to kill homotopy groups). Using more sophisticated methods, one can 
realize String $(V)$ as a topological group. An explicit realization of $\operatorname{String}(V)$ as an (infinite dimensional) topological group is described in [28].

An alternative point of view is to consider $\operatorname{String}(V)$ as the total space over a certain $S^{1}$-gerbe over $\operatorname{Spin}(V)$. This has the advantage of being a "finite dimensional" object that can be studied using ideas from differential geometry (see for example [10]).

Let $M$ be a smooth manifold of dimension $n$. Choosing a Riemannian metric on $M$, we can reduce the structure group of the tangent bundle of $M$ to $\mathrm{O}(V)$. An orientation (spin structure, string structure) on $M$ is a further reduction of the structure group of the tangent bundle $T_{M}$ to $\mathrm{SO}(V)(\operatorname{Spin}(V), \operatorname{String}(V))$. The manifold $M$ admits an orientation if and only if the first Stiefel-Whitney class $w_{1}(M)$ vanishes. An orientation of $M$ can be lifted to a spin structure if and only if the second Stiefel-Whitney class $w_{2}(M)$ vanishes. Likewise, a spin structure on $M$ extends to a string structure if and only if a certain characteristic class $p \in \mathrm{H}^{4}(M ; \mathbf{Z})$ vanishes. The characteristic class $p$ has the property that $2 p$ coincides with the first Pontryagin class $p_{1}(M)$, though $p$ itself is well-defined only after a spin structure on $M$ has been chosen.

Let $A$ be a multiplicative cohomology theory. Then $A$ determines a (dual) homology theory, which we will also denote by $A$. A class $\eta \in A_{n}(M)$ is an $A$-orientation of $M$ provided that cap product by $\eta$ induces an isomorphism

$$
A^{*}(*) \rightarrow A_{n-*}(M, M-\{m\})
$$

for every point $m \in M$. In this case, we will say that $M$ is $A$-oriented and that $\eta$ is the $A$-fundamental class of $M$.

In the case where $A$ is ordinary integral homology, giving an $A$-orientation of $M$ is equivalent to reducing the structure group of the tangent bundle of $M$ to $\mathrm{SO}(V)$. Similarly, giving a spin structure on $M$ allows one to define an orientation of $M$ with respect to complex $K$-theory (even with respect to real $K$-theory). The idea is that $K$-homology classes can be represented by elliptic differential operators; the appropriate candidate for the fundamental class of $M$ is the Dirac operator, which is well-defined once $M$ has been endowed with a spin structure. In this section, we would like to discuss the "elliptic" analogue of this result: if $M$ is a string manifold, then $M$ has a canonical orientation with respect to elliptic cohomology.

The relationship between elliptic cohomology orientations of a manifold and string structures goes back to the work of Witten (see [30]). Heuristically, the elliptic cohomology of a space $M$ can be thought of as the $S^{1}$-equivariant $K$-theory of the loop space $L M$. To obtain an elliptic cohomology orientation of $M$, one wants to write down the Dirac equation on $L M$. Witten computed the index of this hypothetical Dirac operator using a localization formula, and thereby defined the Witten genus $w(M) \in \mathbf{Z}((q))$ of the manifold $M$, having the property that the coefficient of $q^{n}$ in $w(M)$ is the $\chi^{n}$-isotypic part of the index of the Dirac operator, where $\chi: S^{1} \rightarrow \mathbf{C}^{*}$ is the identity character. Moreover, he made the following very suggestive observation: if $p_{1}(M)=0$, then $w(M)$ is the $q$-expansion of a modular form.

Remark 5.7. The statement that the elliptic cohomology of $M$ is given by the $S^{1}$-equivariant $K$-theory of the loop space $L M$ is only heuristically true. However, it is true in the "limiting" case where we consider elliptic cohomology near $\infty$, and we consider only "small" loops in $M$. As we saw in $\S 4.3$, in a formal disk around $\infty$, elliptic cohomology may be identified with $K((q))$. Moreover, $K((q))(M)$ is a completion of $K_{S^{1}}(M)$, where we identify $M$ with the subset of $L M$ consisting of constant loops (so that $S^{1}$ acts trivially on this space). The results described in $\S 5.2$ may be considered as a less trivial illustration of the same principle.

In order to study the problem of orienting various classes of manifolds more systematically, it is convenient to pass to the limit by defining

$$
\begin{aligned}
\mathrm{O} & =\mathfrak{\operatorname { l i m }}_{\longrightarrow}\left\{\mathrm{O}\left(\mathbb{R}^{d}\right)\right\}_{d \geq 0} \\
\mathrm{SO} & =\mathrm{lim}_{\mathrm{l}}\left\{\mathrm{SO}\left(\mathbb{R}^{d}\right)\right\}_{d \geq 0} \\
\mathrm{Spin} & =\underline{\lim }\left\{\operatorname{Spin}\left(\mathbb{R}^{d}\right)\right\}_{d \geq 0} \\
\text { String } & =\underline{\lim }\left\{\operatorname{String}\left(\mathbb{R}^{d}\right)\right\}_{d \geq 0}
\end{aligned}
$$


Let $G$ denote any of these groups (though much of what we say below applies to other structure groups as well). If $M$ is a smooth manifold of any dimension, we will say that a $G$-structure on $M$ is a reduction of the structure group of the stabilized tangent bundle of $M$ from $\mathrm{O}$ to $G$. In this case, we will say that $M$ is a G-manifold.

Fix a topological group $G$ with a map $G \rightarrow \mathrm{O}$, and consider the class of $G$-manifolds: that is, smooth manifolds $M$ whose structure group has been reduced to $G$. To this data, one can associate a Thom spectrum $M G$. Roughly speaking, one defines $M G_{n}(X)$ to be the set of bordism classes of $n$-dimensional $G$-manifolds $M$ equipped with a map $M \rightarrow X$. In particular, if $M$ is a $G$-manifold of dimension $n$, there is a canonical element $\eta \in M G_{n}(M)$; this is an orientation of $M$ with respect to the cohomology theory $M G$. One can view $M G$ as the universal cohomology theory for which every $G$-manifold has an orientation. More generally, if $A$ is an arbitrary cohomology theory, then equipping every $G$-manifold $M$ with an $A$-orientation is more or less equivalent to giving a map $s: M G \rightarrow A$. In good cases, the cohomology theory $A$ will be represented by an $E_{\infty}$-ring, and $s$ will be a map of $E_{\infty}$-rings: roughly speaking, this may be thought of as asserting that the orientations on $G$-manifolds determined by $s$ are compatible with the formation of products of $G$-manifolds.

The statements we made earlier, regarding orientations of manifolds with respect to ordinary homology and $K$-theory, can be reinterpreted as asserting the existence of certain natural maps

$$
\begin{gathered}
\mathrm{MSO} \rightarrow \mathbf{Z} \\
\mathrm{MSpin} \rightarrow \mathrm{KO}
\end{gathered}
$$

between $E_{\infty}$-rings. Similarly, the string orientability of elliptic cohomology can be formulated as the existence of an $E_{\infty}$-map

$$
\sigma: \text { MString } \rightarrow \text { tmf . }
$$

The map $\sigma$ is sometimes called the topological Witten genus; when composed with the map tmf $\rightarrow K((q))$ and evaluated on a string manifold $M$, it reduces to the ordinary Witten genus described above.

The existence of the map $\sigma$ is known, thanks to the work of Ando, Hopkins, Rezk, Strickland, and others. We refer the reader to [4] for a discussion of the problem in a somewhat simpler setting. We will sketch here an alternative construction of the map $\sigma$, using the theory of 2-equivariant elliptic cohomology sketched in $\S 5.1$. For simplicity, we let $E \rightarrow \operatorname{Spec} A$ be an oriented elliptic curve over an $E_{\infty}$-ring $A$; we will construct a natural map $\sigma_{A}$ : MString $\rightarrow A$. The map $\sigma$ itself is obtained by passing to the (homotopy) inverse limit.

We first rephrase the notion of an orientation from a point of view which is more readily applicable to our situation. Given a group homomorphism $s: G \rightarrow \mathrm{O}$, we obtain a map of classifying spaces $B G \rightarrow B \mathrm{O}$, which we may think of as a (stable) vector bundle over $B G$. This vector bundle determines a spherical fibration over $B G$, which we will view as a bundle of invertible $S$-modules over $B G$. Given an $E_{\infty}$-ring $A$, we may tensor with $A$ to obtain a bundle of $A$-modules over $B G$; let us denote this bundle by $\mathcal{A}_{s}$. To give a map of $E_{\infty}$-rings $M G \rightarrow A$ is equivalent to giving a trivialization of the local system $\mathcal{A}_{s}$, which is suitably compatible with the group structure on $B G$. To simplify the discussion, we will focus only on trivializing $\mathcal{A}_{s}$; the compatibility with the group structure is established by a more careful application of the same ideas.

Let us now specialize to the case $G=$ Spin, and suppose that we are given an oriented elliptic curve over $A$. The theory of equivariant elliptic cohomology associates to the compact Lie group $\operatorname{Spin}(n)$ a derived $A$-scheme $M_{\text {Spin }}$. Moreover, the functoriality of the construction gives a map of topological spaces

$$
\phi: B \operatorname{Spin}(n) \simeq \operatorname{Hom}(*, B \operatorname{Spin}(n)) \rightarrow \operatorname{Hom}\left(\operatorname{Spec} A, M_{\text {Spin }}\right) .
$$

Let $l: B \operatorname{Spin}(n) \rightarrow K(\mathbf{Z}, 4)$ be the canonical generator; then the theory of 2-equivariant elliptic cohomology associates to $l$ a line bundle $\mathcal{L}_{l}$ over $M_{\text {Spin }}$. Thus, for every $A$-valued point of $M_{\text {Spin }}$, we get an invertible $A$-module. The morphism $\phi$ therefore gives a local system of invertible $A$-modules on $B \operatorname{Spin}(n)$; let us denote it by $\mathcal{A}_{\phi}$.

The existence of the string orientation on elliptic cohomology rests on the following comparison result, which we will not prove here: 
Theorem 5.2. Let $s: \operatorname{Spin}(n) \rightarrow \mathrm{O}$ be the natural homomorphism and let $E \rightarrow \operatorname{Spec} A$ be an oriented elliptic curve over an $E_{\infty}$-ring $A$. There is a canonical isomorphism

$$
\mathcal{A}_{\phi} \simeq \mathcal{A}_{s}
$$

of local systems of invertible A-modules over B $\operatorname{Spin}(n)$.

To provide $A$ with a string orientation, we need to prove that $\mathcal{A}_{s}$ becomes trivial after pulling back along the map $B \operatorname{String}(n) \rightarrow B \operatorname{Spin}(n)$. By Theorem 5.2, it will suffice to prove that $\mathcal{A}_{\phi}$ becomes trivial after pullback to $B$ String $(n)$. The theory of 2-equivariant elliptic cohomology provides a commutative diagram

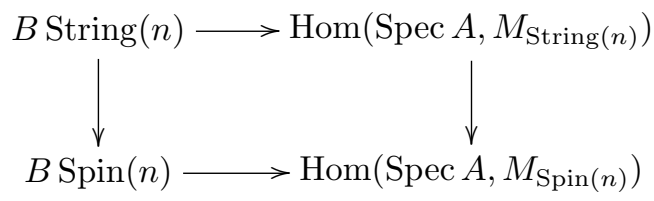

Consequently, to prove that $\mathcal{A}_{\phi}$ becomes trivial after pullback to $B \operatorname{String}(n)$, it suffices to prove that $\mathcal{L}_{l}$ becomes trivial after pullback along the map of derived schemes $M_{\operatorname{String}(n)} \rightarrow M_{\operatorname{Spin}(n)}$. But $M_{\operatorname{String}(n)}$ is precisely the total space of the principal $\mathbb{G}_{m}$-bundle underlying $\mathcal{L}_{l}$ : in other words, it is universal among derived schemes over $M_{\operatorname{Spin}(n)}$ over which $\mathcal{L}_{l}$ has a trivialization.

Remark 5.8. The argument given above not only establishes the existence of the string orientation MString $\rightarrow$ $A$; it also explains why the covering $\operatorname{String}(n) \rightarrow \operatorname{Spin}(n)$ arises naturally when one considers the problem of finding orientations with respect to elliptic cohomology.

\subsection{Higher Equivariance}

The purpose of this section is to place the theory of 2-equivariant elliptic cohomology, which we discussed in $\S 5.1$, into a larger context.

Let $A$ be an even, periodic cohomology theory, and suppose that $A(*)=k$ is a field. In this case, there are not many possibilities for the formal group $\widehat{\mathbb{G}}=\operatorname{Spf} A\left(\mathbf{C P}^{\infty}\right)$. If $k$ is of characteristic zero, any 1-dimensional formal group over $k$ is isomorphic to the formal additive group; correspondingly, $A$ is necessarily equivalent to periodic ordinary cohomology with coefficients in $k$. If $k$ is of characteristic $p$, then the formal group $\mathbb{G}$ is classified up to isomorphism (over the algebraic closure $\bar{k}$ ) by a single invariant $1 \leq n \leq \infty$. The invariant $n$ is called the height of $\mathbb{G}$, and may be thought of as a measure of the size of the $p$-torsion subgroup $\mathbb{G}[p] \subseteq \mathbb{G}$. By convention, we say that the additive group in characteristic zero has height zero (since it has no nontrivial $p$-torsion).

To a formal group $\mathbb{G}$ of height $n$ over an algebraically closed field $k$ of characteristic $p$, one can associate an (essentially unique) even, periodic cohomology theory. This cohomology theory is called Morava K-theory and denoted by $K(n)$.

The Morava $K$-theory of Eilenberg-Mac Lane spaces has been computed by Ravenel and Wilson (see [25]). In particular, they show that for $m>n$, the natural map

$$
K(n)^{*}(*) \rightarrow K(n)^{*}(K(\mathbf{Z} / p \mathbf{Z}, m))
$$

is an isomorphism. In other words, the Morava $K$-theory $K(n)$ cannot tell the difference between the space $K(\mathbf{Z} / p \mathbf{Z}, m)$ and a point. We may informally summarize the situation by saying that $K(n)$ does not "see" the homotopy groups of a space $X$ in dimensions larger than $n$. Of course, this is not literally true: however, it is true provided that the homotopy groups of $X$ satisfy certain finiteness properties.

If $\mathbb{G}$ is a formal group over a commutative ring $R$, then it need not have a well-defined height; however, it has a height when restricted to each residue field of $R$. We will say that an cohomology theory $A$ has $h e i g h t \leq n$ if it is even, weakly periodic, and the associated formal group has height $\leq n$ at every point. Our 
discussion of Morava $K$-theory can be generalized as follows: if $A$ is a cohomology theory of height $\leq n$, then $A$ only "sees" the first $n$ homotopy groups of a space $X$. As we remarked above, this is not true in general, but it is true provided that we make suitable finiteness assumptions on $X$.

Let us first consider the case of a cohomology theory $A$ of height $\leq 0$. Then $R=A(*)$ is an algebra over the field $\mathbf{Q}$ of rational numbers, and $A$ is automatically a periodic variant of ordinary cohomology with coefficients in $R$. It follows that $A(X)$ is insensitive to the homotopy groups of $X$, provided that those homotopy groups are finite. For spaces with finite homotopy groups, $A(X)$ simply measures the number of connected components of $X$.

We can get more information by considering cohomology theories of height $\leq 1$. The prototypical example is complex $K$-theory. The above discussion indicates that, under suitable finiteness hypotheses, $K(X)$ should be sensitive only to the fundamental groupoid of $X$. In other words, the only really interesting $K$-group to compute is $K(B G)$, where $G$ is a finite group. However, this example turns out to be quite interesting: there is a natural map $\eta: \operatorname{Rep}(G) \rightarrow K(B G)$, defined for any finite group $G$. The Atiyah-Segal completion theorem asserts that $\eta$ is not far from being an isomorphism: it realizes $K(B G)$ as the completion of $\operatorname{Rep}(G)$ with respect to the augmentation ideal consisting of virtual representations having virtual dimension zero.

There are many respects in which $\operatorname{Rep}(G)$ is a better behaved object than $K(B G)$ : for example, it is a finitely generated abelian group, while $K(B G)$ is not. Moreover, there is a moral sense in which $K(B G)$ "ought to be" $\operatorname{Rep}(G)$ : the fact that $\eta$ is not an isomorphism is somehow a technicality. We can regard equivariant $K$-theory as a way of formally "defining away" the technicality. Namely, the equivariant $K$ group $K_{G}(*)$ is a refined version of $K(B G)$, which coincides with $\operatorname{Rep}(G)$ by definition.

One might ask if there are other examples of spaces $X$ for which $K(X)$ is in need of refinement. According to the discussion above, the answer is essentially no: $K(X)$ should only be sensitive to the fundamental groupoid of $X$, so that the general "expected answer" for $K(X)$ is the representation $\operatorname{ring} \operatorname{Rep}\left(\pi_{1} X\right)$ (provided that $X$ is connected).

When we consider cohomology theories of higher height, the above argument breaks down. Let Ell denote an elliptic cohomology theory, necessarily of height $\leq 2$. Following the above discussion, we should imagine that $\operatorname{Ell}(X)$ is sensitive to the first two homotopy groups of a space $X$. Consequently, it is most interesting to compute $\operatorname{Ell}(X)$ when $X$ is a connected space satisfying $\pi_{1} X=G, \pi_{2} X=A$, and $\pi_{n} X=0$ for $n>2$. In the above discussion, we assumed that the groups $G$ and $A$ were finite. However, by analogy with $K$-theory, we should also allow the case where $G$ and $A$ are compact Lie groups (so that $X$ is allowed to be a space like $B G$, or the classifying space of a circle gerbe over $G$ ). By analogy with $K$-theory, one might guess that there is an "expected" answer for $\operatorname{Ell}(X)$, and that $\operatorname{Ell}(X)$ is related to this expected answer by some sort of completion result in the spirit of the Atiyah-Segal theorem. This prediction turns out to be correct: there is an expected answer, which is dictated by the geometry of elliptic curves. Moreover, as with equivariant $K$-theory, one can build a coherent and useful theory out of the expected answers. This is the theory of 2-equivariant elliptic cohomology which we discussed in $\S 5.1$.

Remark 5.9. There is no reason to stop at height 2. Given an $E_{\infty}$-ring $A$ and an oriented $p$-divisible group $\mathbb{G}$ over $A$ of height $n$, one can construct a theory of n-equivariant $A$-cohomology. Namely, let $X$ be a space whose homotopy groups are all finite $p$-groups, vanishing in dimension $>n$. Then there is a natural procedure for using $\mathbb{G}$ to construct a spectrum $A_{X}$, which can be thought of as the "expected answer" for $A(X)$. Moreover, there is a map $A_{X} \rightarrow A(X)$ which can be described by an Atiyah-Segal completion theorem. The significance of these "expected answers" is not yet clear, but they are closely related to the generalized character theory of $[16]$.

\subsection{Elliptic Cohomology and Geometry}

In this paper, we have discussed elliptic cohomology from an entirely algebraic point of view. In doing so, we have ignored what is perhaps the most interesting question of all: what is elliptic cohomology? In other words, given a topological space $X$, what does it mean to give an elliptic cohomology class $\eta \in \operatorname{tmf}(X)$ ? No satisfactory answer to this question is known, as of this writing. However, a number of very interesting ideas 
have been put forth. We saw in $\S 5.2$ that equivariant elliptic cohomology is related to the theory of loop group representaions. Graeme Segal has suggested that elliptic cohomology should bear some relationship to Euclidean field theories (see [27]). Building on his ideas, Stolz and Teichner have proposed that the classifying space for elliptic cohomology might be interpreted as a moduli space for supersymmetric quantum field theories. To support their view, they show in [28] that supersymmetry predicts that the coefficients in the $q$-expansion of the partition function of such a theory should be integral. Alternative speculations on the problem can be found in [18] and [7], among other places.

Our moduli-theoretic interpretation of elliptic cohomology has the advantage of being well-suited to proving comparison results with other theories. Suppose that we are given some candidate cohomology theory $A$, which we suspect is equivalent to elliptic cohomology. For simplicity, we will assume that $A$ is a candidate for the elliptic cohomology theory associated to an elliptic curve over an affine base Spec $\pi_{0} A$ (the non-affine case can be treated as well, but requires a more elaborate discussion). We will suppose that $A$ enjoys all of the good formal properties of elliptic cohomology: namely, that it is representable by an $E_{\infty}$-ring, and that it has well-behaved equivariant and 2-equivariant analogues.

To relate $A$ to elliptic cohomology, we would like to produce a map $f: \operatorname{tmf} \rightarrow A$. Better yet, we would like to have a map $\operatorname{Spec} A \rightarrow \mathcal{M}^{\text {Der }}$ : we can then obtain $f$ by considering the induced map on global sections of the structure sheaves. To provide such a map, we need to give an oriented elliptic curve $E \rightarrow \operatorname{Spec} A$. We will proceed under the assumption that $E$ exists, and explain how to reconstruct it from the cohomology theory $A$.

We wish to produce $E$ not just as a derived scheme, but as an oriented $A$-group. Equivalently, for every lattice $\Lambda$, we want to construct the abelian variety $E^{\Lambda}=\operatorname{Hom}(\Lambda, E)$, and we want the construction to be functorial in $\Lambda$. Let $T=\operatorname{Hom}\left(\Lambda, S^{1}\right)$ denote the Pontryagin dual of $\Lambda$. If $E^{\Lambda}$ were affine, we would expect to recover it as $\operatorname{Spec} A_{T}(*)$. Unfortunately, abelian varieties are not affine, so we must work a bit harder. To this end, select a map $q: B T \rightarrow K(\mathbf{Z}, 4)$ which classifies a positive definite quadratic form on $\Lambda^{\vee}$. The level $q$ determines an ample line bundle $\mathcal{L}_{q}$ on $E^{\Lambda}$. Moreover, the global sections of the $k$ th power of $\mathcal{L}_{q}$ should be given by $T$-equivariant $A$-cohomology at level $k q$ : we can make sense of this $A$-module in virtue of the assumption that we have a good 2-equivariant theory. We can now assemble these modules of sections, for varying $k \geq 0$, into a graded $E_{\infty}$-ring $R_{\bullet}$, and attempt to recover $\mathcal{E}^{\Lambda}$ as the projective spectrum of $R_{\bullet}$.

Of course, the above construction will only work to produce an abelian variety over $\operatorname{Spec} A$ if certain conditions are met. These conditions can be reduced to certain computations: for example, one must show that if $T$ is a torus and $q: B T \rightarrow K(\mathbf{Z}, 4)$ classifies a positive definite quadratic form of discriminant $d$, then the $T$-equivariant $A$-cohomology of a point, at level $q$, is a locally free $A$-module of rank $d$. Supposing that this and other algebraic conditions are satisfied, the above construction will give us an elliptic curve $E \rightarrow \operatorname{Spec} A$. Moreover, by comparing equivariant $A$-cohomology with Borel-equivariant $A$-cohomology, we can supply $E$ with a preorientation $\sigma: \mathbf{C P}^{\infty} \rightarrow E(A)$. The condition that $\sigma$ be an orientation is again a matter of computation: essentially, we would need to show that an appropriate version of the AtiyahSegal completion theorem holds, at least locally on the elliptic curve $E$. Provided that all of the necessary computations yield favorable results, we obtain an oriented elliptic curve $E \rightarrow \operatorname{Spec} A$, which is classified by the desired map Spec $A \rightarrow \mathcal{M}^{\text {Der }}$.

Unfortunately, our algebraic perspective does not offer any insights on the problem of where to find such a cohomology theory in geometry. Nevertheless, it seems inevitable that a geometric understanding of elliptic cohomology will eventually emerge. The resulting interaction between algebraic topology, number theory, mathematical physics, and classical geometry will surely prove to be an excellent source of interesting mathematics in years to come.

\section{References}

1. Adams, J.F. Stable Homotopy and Generalised Homology. University of Chicago Press, 1974.

2. Adams, J.F. A variant of E.H. Brown's representability theorem. Topology 10, 1971, 185-198.

3. Ando, M. Power operations in elliptic cohomology and representations of loop groups. Transactions of the American Mathematical Society 352, 2000, pp. 5619-5666. 
4. Ando, M., Hopkins, M. and N. Strickland. Elliptic spectra, the Witten genus, and the theorem of the cube. Inventiones Mathematicae 146 (2001), no.3, 595-687.

5. Atiyah, M. and R. Bott. On the periodicity theorem for complex vector bundles. Acta Mathematica 112, 1964, pp. 229-247.

6. Atiyah, M.F. and G.B. Segal. Equivariant K-theory and completion. J. Differential Geometry 3, 1969, 1-18.

7. Baas, N.A., Dundas, B.I. and J. Rognes. Two-vector bundles and forms of elliptic cohomology. Topology, geometry, and quantum field theory, London Mathematical Soceity Lecture Note Series 308. Cambridge University Press, Cambridge, 2004, pp. 18-45.

8. Behrens, M. and T. Lawson. Topological Automorphic Forms. Available on the mathematics archive as math.AT/0702719.

9. Breen, L. Fonctions thêta et théorème du cube. Lecture Notes in Mathematics 980, Springer-Verlag, Berlin, 1983.

10. Brylinski, J-L. Loop spaces, characteristic classes, and geometric quantization. Progress in Mathematics 107 , Birkhauser Boston, Inc. Boston, MA, 1993.

11. Eilenberg, S. and N.E. Steenrod. Axiomatic approach to homology theory. Proc. Nat. Acad. Sci. U.S.A. 31, 1945, $117-120$.

12. Elmendorf, A.D., Kriz, I., Mandell, M.A. and J.P. May. Rings, modules, and algebras in stable homotopy theory. Mathematical Surveys and Monographs, 47. American Mathematical Society, Providence, RI, 1997.

13. Freed, D., Hopkins, M. and C. Teleman. Twisted $K$-theory and loop group representations. Available on archive as math.AT/0312155.

14. Friedman, R., Morgan, J.W. and E. Witten. Principal G-bundles over elliptic curves. Mathematical Research Letters 5, 1998, no. 1-2, pp. 97-118.

15. Fulton, W. Introduction to toric varieties. Annals of Mathematics Studies 131. Princeton University Press, Princeton, NJ, 1993.

16. Hopkins, M., Kuhn, N. and D. Ravenel. Generalized group characters and complex oriented cohomology theories. Journal of the American Mathematical Society 13 (2000), no. 3, 553-594.

17. Hovey, M., Shipley, B. and J. Smith. Symmetric spectra. Journal of the American Mathematical Society 13 no.1, 2000, pp. 149-208.

18. Hu, P. and I. Kriz. Conformal field theory and elliptic cohomology. Advances in Mathematics 189 (2004), no.2., 325-412.

19. Landweber, P. Homological Properties of Comodules over $\mathrm{MU}_{*}(\mathrm{MU})$ and $\mathrm{BP}_{*}(\mathrm{BP})$. American Journal of Mathematics, Volume 98, No. 3, pp. 591-610.

20. Lurie, J. Derived algebraic geometry. Unpublished MIT PhD dissertation, 2004.

21. Lurie, J. Elliptic cohomology. In preparation.

22. Lurie, J. Higher topos theory. To be published by the Princeton Univesity Press; presently available for download at http://www.math.harvard.edu/ lurie/.

23. Milnor, J.W. and J.D. Stasheff. Characteristic classes. Annals of Mathematics Studies No. 76. Princeton University Press, Princeton, NJ, 1974.

24. Pressley, A. and G. Segal. Loop groups. Oxford Science Publications. The Clarendon Press, Oxford University Press, New York, 1986.

25. Ravenel, D.C. and W.S. Wilson. The Morava K-theories of Eilenberg-Mac Lane spaces and the Conner-Floyd conjecture. American Journal of Mathematics 102, 1980, no. 4., pp. 691-748.

26. Rezk, C. Notes on the Hopkins-Miller Theorem. Homotopy Theory via Algebraic Geometry and Group Representation Theory. Contemporary Mathematics 220, 313-366.

27. Segal, G. Elliptic cohomology (after Landweber-Stong, Ochanine, Witten and others). Asterisque 161-162, 1988, Expose No. 695, 4, pp. 187-201.

28. Stolz, S. and P. Teichner. What is an elliptic object? Topology, geometry, and quantum field theory. London Mathematical Society Lecture Note Series 308, Cambridge University Press, Cambridge, 2004, pp. $247-343$.

29. Toën, B. and G. Vezzosi. From HAG to DAG: derived moduli stacks. Axiomatic, enriched, and motivic homotopy theory, NATO Science Series II: Mathematics, Physics, and Chemistry 131, 173-216.

30. Witten, E. Elliptic genera and quantum field theory. Communications in Mathematical Physics 109, 1987, no.4, pp. 525-536. 